\title{
Genetics in Medicine
}

\section{Non-invasive prenatal diagnosis by genome-wide haplotyping of cell-free plasma DNA --Manuscript Draft--}

\begin{tabular}{|c|c|}
\hline Manuscript Number: & GIM-D-19-00723R1 \\
\hline Article Type: & Article \\
\hline Section/Category: & Reproductive Genetics \\
\hline Keywords: & Non-invasive prenatal diagnosis; cell free DNA; haplotyping; monogenic diseases \\
\hline Corresponding Author: & $\begin{array}{l}\text { Joris R. Vermeesch, Ph.D. } \\
\text { Centre for Human Genetics, KU Leuven } \\
\text { Leuven, BELGIUM }\end{array}$ \\
\hline First Author: & Huiwen Che, M.S. \\
\hline \multirow[t]{13}{*}{ Order of Authors: } & Huiwen Che, M.S. \\
\hline & Darine Villela \\
\hline & Eftychia Dimitriadou \\
\hline & Cindy Melotte \\
\hline & Nathalie Brison \\
\hline & Maria Neofytou \\
\hline & Kris Van Den Bogaert \\
\hline & Olga Tsuiko \\
\hline & Koen Devriendt \\
\hline & Eric Legius \\
\hline & Masoud Zamani Esteki \\
\hline & Thierry Voet \\
\hline & Joris Robert Vermeesch \\
\hline Manuscript Region of Origin: & BELGIUM \\
\hline Abstract: & $\begin{array}{l}\text { Purpose Whereas non-invasive prenatal testing for aneuploidies (NIPT-A) is widely } \\
\text { implemented, there is an increasing need for universal approaches for noninvasive } \\
\text { prenatal testing for monogenic diseases (NIPT-M). Here, we present a cost-effective, } \\
\text { generic cell-free fetal DNA (cffDNA) haplotyping approach to scan the fetal genome for } \\
\text { the presence of inherited monogenic diseases. Methods Families participating in the } \\
\text { preimplantation genetic testing for monogenic disorders (PGT-M) program were } \\
\text { recruited for this study. } 250000 \text { SNPs captured from maternal plasma DNA along with } \\
\text { genomic DNAs from family members were massively parallel sequenced. Parental } \\
\text { genotypes were phased via an available genotype from a close relative, and the fetal } \\
\text { genome-wide haplotype and copy number were determined using cffDNA haplotyping } \\
\text { analysis based on estimation and segmentation of fetal allele presence in the maternal } \\
\text { plasma. Results In all families tested, mutational profiles from cffDNA haplotyping are } \\
\text { consistent with embryo biopsy profiles. Genome-wide fetal haplotypes are on average } \\
97 \% \text { concordant with the newborn haplotypes and embryo haplotypes. Conclusion We } \\
\text { demonstrate that genome-wide targeted capture and sequencing of polymorphic SNPs } \\
\text { from maternal plasma cell free (cfDNA) allows haplotyping and copy number profiling } \\
\text { of the fetal genome during pregnancy. The method enables the accurate } \\
\text { reconstruction of the fetal haplotypes and can be easily implemented in clinical } \\
\text { practice. }\end{array}$ \\
\hline
\end{tabular}


To Dr Robert D Steiner

Editor-in-Chief

Genetics in Medicine

Ref: GIM-D-19-00723

Dear Dr. Steiner,

Thank you for your email regarding revision of the manuscript "Non-invasive prenatal diagnosis by genome-wide haplotyping of cell-free plasma DNA". We have carefully read the comments and revised the manuscript accordingly.

We would like to thank the reviewer for thoughtful reading the manuscript and for their constructive suggestions. Our responses are in blue given in a point-by-point manner (the reviewers' comments are in italics).

Sincerely,

Joris R. Vermeesch, Ph.D.

Professor of Laboratory of Cytogenetics and Genome Research

Head of the Department of Human Genetics, KU Leuven

Herestraat 49, box 602, 3000 Leuven, Belgium

e-mail: joris.vermeesch@uzleuven.be 



\section{Response to the reviewers}

Reviewer \#1: The potential application of NIPD to follow up the results of PGT is exciting, as increasing number of PGT couples are reluctant to follow the current recommendations for invasive prenatal diagnosis; thus this manuscript presents a clinical scenario and experimental details of such a practical application. The work is well written, and will be of interest to a wide audience.

- Perhaps the authors could expand in a more detail the accuracy of a calculation of fetal fraction, and a lower fetal fraction limit for the conclusion.

We added discussion of lower fetal fraction limit on page 18 line 369-374.

- It will be also useful if authors could discuss any additional factors that may impact the accuracy of NIPD. One of such factors that creates a concern is a genetic instability in preimplantation development that may affect the accuracy of testing. However, these are minor suggestions to consider for discussion.

Regarding genetic instability of preimplantation embryos, we have recently demonstrated that the impact of embryonic aneuploidy seems to diminish during prenatal development (Zamani-Esteki et al., 2019 Nature Med), further minimizing the concern that embryo genetic instability can be a confounding factor for cfDNA haplarithmisis. We commented it on page 19 line 377-385.

Reviewer \#2: Huiwen Che and colleagues describe a method they devised for genome-wide haplotyping on cell-free DNA, to allow concurrent diagnosis of aneuploidies with inheritance of known familial pathogenic variants in recessive, dominant and X-linked disorders. They use as underlying principle for their approach a method which they named "haplarithmisis" and that was original developed for $P G T$ on in vitro-fertilized preimplantation embryos.

This is primarily a proof of concept paper with initial technical validation, but the sample size is fairly small (9 families and one "artificially mixed" aneuploidy sample. Overall the paper is well written and clear, supporting tables and figures (including in supplemental data) are appropriate and informative, with some details that need to be addressed (see below).

It is not the first demonstration that this type of data can be obtained from cffDNA, and the approach labeled "haplarithmisis" and determination of Fetal Allele Ratio (FAR) is essentially based on RHDO of multiple SNPs at once, with some refinements to optimize analysis by optimizing which SNPs are used in the analysis (addition of informative type 4 SNPs) and pairing with circular binary segmentation that allows them to overcome noise and an have estimate of recombination sites in the 
haplotypes.

The conceptual innovation in this paper results from the combined aneuploidy and monogenic disease testing possibilities on a more genome-wide basis using a less costly and faster method than those previously published that also avoids the requirement for disease-specific work-up.

Although the numbers are small, the validation for the haplotyping on pregnancies conceived after PGT-M, wherein both embryo and newborn haplotypes are available for comparison is elegant. I am less impressed with the fairly limited approach to the validation of the aneuploidy detection, which is entirely based on one mixing experiment with spike-in samples, since they always have the limitations that fragmentation is not identical to that of naturally occurring cfDNA. The study would have more value if a few cases where analysis is done on cffDNA from aneuploid pregnancies with comparison of data from newborn genomic DNA/karyotype (or from products of conception results). The ability to determine parental origin of the aneuploidy if validated is an advantage of this method.

We thank the Reviewer for an extensive evaluation and agree that mixing experiment with spike-ins is a limitation, when it comes to validation of aneuploidy detection. Unfortunately, we were not able to perform direct comparison of aneuploidy detection in cffDNA and affected newborn or product of conception, as in the framework of this project we did not have an aneuploidy clinical case. However, we are keeping running the clinical study to collect samples to further validate the method. We foresee that we can have access to aneuploidy cases to overcome the limitations of 'artificial' sample.

I have the following additional detailed comments and suggestions:

- The abstract is clear and reflects the data and conclusions of the manuscript accurately. I like the introduction of NIPT-A and NIPT-M, as terms that nicely parallel PGT-A and PGT-M. Maybe authors could consider including NIPT-SR (or NIPT-CN or "copy number"?) in their terminology as well and elaborate on this suggested terminology in the discussion some more?

We address the point in the discussion on page 16 line 319-322.

- Introduction: on P 3, first paragraph: NIPT-A indeed resulted in a significant drop on invasive procedures, however the word "unnecessary", which is an interpretation, should be removed from this sentence. Concern has been raised that while procedures for aneuploidy testing have gone down, the side effect is that there is also a decline in procedures for diagnoses that are not found by NIPT-A and that would have been detected by a diagnostic procedure. 
We removed "unnecessary" from the sentence on page 3 line 53.

- Introduction: P4, last line (and also in methods): can authors define the word "haplarithmisis". I realize the method has been previously published and is briefly described, but it will help readers to understand what the basis for this unusual terminology is.

We added explanations for haplarithmisis on page 5 line 83-85.

- I suggest that for all relevant figures you consider different color combinations to designate the different parental SNP subcategories and haplotype blocks besides red and blue. Sometimes red blue differentiates two different paternal haplotypes of the same parent and sometimes it differentiates maternal versus paternal in the fetal DNA.

We clarified the color pattern in the figure legend.

- Figure 3: Figure 3a is a little hard to follow. Consider more clarification in legend or revision of this panel. Figures $3 c$ and $3 d$ are too small. I had to significantly enlarge these on the computer monitor to actually see the two blue and two red tracks for each chromosome. It is impossible to see this on the printed version. This should be corrected (same applies for similar figures in supplemental data).

We moved Figure 3a and 3b to Supplementary Figure S5a and S5b and added supplementary figure legend for S5a. We rearranged Figure 3 and Supplementary Figure S6 to present figures properly.

- I would like to see more clearly described if the actual variant (wild-type versus carrier) was detected accurately in addition to correct haplotype establishment. The authors must have some data on this.

The variants of the clinical samples are not in the target design and thus unfortunately we do not have the information of the allele counts on the specific variant locus. We expect that if actual variants happen to be within the capture design, we can obtain locus-specific information to support haplotyping results.

- There should be some comment in the discussion how the $97 \%$ concordance with embryo and newborn haplotypes would affect clinical utility if introduced for clinical application.

We commented the clinical utility of the method in the discussion on page 18 line 361-365. 
- In the discussion, the authors state that their method is better than working up individual cases and doing targeted cfDNA analysis for familial variants. That statement should be supported by a discussion that considers comparison of cost, time estimates to results, accuracy, and the limitations of their new method. In particular, bespoke testing has been shown to be diagnostic (not screening) in certain studies while their assay seems less accurate for maternally inherited variants and has problems if there is meiotic recombination near a pathogenic variant.

We expanded detailed discussion regarding the advantage and limitations of the method on page 16 line 324-344 and page 17 line 346-385. The maternally inherited variants show lower accuracy compared with paternally inherited variants due to the maternal background DNA in plasma samples. The haplotyping of maternal inheritance is also largely affected by fetal fraction. If the variant lies in meiotic recombination region, the method cannot reach a conclusive result and invasive test is required.

- Considering they also suggest this as a potential non-invasive follow-up assay for PGT-M confirmation, an accurate test to confirm would be desired. I would be concerned that for an individual case, a specific error or limitation in a PGT-M assay done by haplarithmisis may well be carried through if haplarithmisis is also used as the basis for the NIPT-M test. Can they comment on this? maybe bespoke non-invasive testing is the way to go.

Computational workflow behind haplarithmisis for PGT-M has been extensively validated during the developmental stage and has been successfully implemented into the routine clinical practice since 2015 (Dimitriadou et al., 2017, Hum Reprod). Although haplotyping-based NIPT-M is based on haplarithmisis, the computation of NIPT-M relies on fetal proportion of cfDNA is independent of single-cell B-allele frequency in PGT-M. The major limiting factor that might prohibit the use of this technology both in PGT-M and NIPT-M is the proximity of the locus of interest to homologous recombination site.

- The other limitation, the inability to detect de novo variants is touched on very briefly but is significant. Have the authors explored their data for the ability to detect de novo variants on the paternal allele? There should be some data as they are in essence similar to type 1 variants.

Although the test allows detection of paternal de novo mutations, the design did not focus on de novo mutations. As suggested by the reviewer, important diseasecausing genes can be included into the capture design in the future and screening for de novo mutations can be incorporated with inherited variants identification. This is commented on page 17 line 354-365. 
- In the discussion, the comment on the 1\% risk for procedure related pregnancy loss for an invasive procedure is an overestimate. Recent data suggest that it is in fact much lower than 1\%, with several papers indicating there is no significant difference between amniocentesis and CVS. This statement must be revised. Please note that there is a more recent meta-analysis by the same authors of reference 37, which itself is incorrectly referenced as it does not say that the risk is $1 \%$.

We removed the incorrect number. The text was rephrased on page 19 line 394, and the reference was updated to Salomon et al., 2019 Ultrasound Obstet Gynecol.

- The authors have a case with maternal CMT1A and comment that it does not interfere with the analysis, but what the resolution of this method would be to detect such CN changes in cfDNA is not addressed. Can they comment on this? Can they model it?

We added maternal copy number profile of this case to the Supplementary Figure 12 and we comment further on this case in the discussion on page 19 line 377380 .

- The comments on carrier screening should qualify that the most desirable approach would be preconception carrier screening followed by PGT-M rather than carrier testing during pregnancy followed by diagnostic testing or NIPT-M

This item was addressed on page 20 line 413-414.

- It would be nice if the discussion could end with a comment about wider clinical validation to determine clinical utility.

We revised the discussion to address the item on page 21 line 420-422. 
1 Non-invasive prenatal diagnosis by genome-wide haplotyping of cell-free plasma DNA

2

3 Huiwen Che $^{1}$, Darine Villela ${ }^{1,2}$, Eftychia Dimitriadou ${ }^{1}$, Cindy Melotte ${ }^{1}$, Nathalie Brison ${ }^{1}$,

4 Maria Neofytou $^{1}$, Kris Van Den Bogaert ${ }^{1}$, Olga Tsuiko ${ }^{1}$, Koen Devriendt ${ }^{1}$, Eric Legius ${ }^{1}$,

5 Masoud Zamani Esteki ${ }^{1,3,4}$, Thierry Voet ${ }^{1}$, Joris Robert Vermeesch ${ }^{1 *}$

6

$7 \quad{ }^{1}$ Center for Human Genetics, KU Leuven, Leuven, Belgium

$8{ }^{2}$ Department of Genetics and Evolutionary Biology, Institute of Biosciences, University of

9 Sao Paulo, Sao Paulo, Brazil

$10{ }^{3}$ Department of Clinical Genetics, Maastricht University Medical Center, Maastricht, The

11 Netherlands

$12{ }^{4}$ Department of Genetics and Cell Biology, GROW School for Oncology and Developmental

13 Biology, Maastricht University, Maastricht, The Netherlands

14

$15 *$ Corresponding author: Joris Robert Vermeesch, Center for Human Genetics, University

16 Hospital Leuven, KU Leuven, Herestraat 49, box 602, 3000 Leuven, Belgium.

17 Telephone: +3216345941

18 Email: joris.vermeesch@kuleuven.be 


\section{ABSTRACT}

\section{Purpose}

22 Whereas non-invasive prenatal testing for aneuploidies (NIPT-A) is widely implemented,

23 there is an increasing need for universal approaches for noninvasive prenatal testing for

24 monogenic diseases (NIPT-M). Here, we present a cost-effective, generic cell-free fetal DNA

25 (cffDNA) haplotyping approach to scan the fetal genome for the presence of inherited

26 monogenic diseases.

\section{Methods}

28 Families participating in the preimplantation genetic testing for monogenic disorders (PGT-

29 M) program were recruited for this study. 250000 SNPs captured from maternal plasma DNA

30 along with genomic DNAs from family members were massively parallel sequenced.

31 Parental genotypes were phased via an available genotype from a close relative, and the fetal genome-wide haplotype and copy number were determined using cffDNA haplotyping analysis based on estimation and segmentation of fetal allele presence in the maternal plasma.

\section{Results}

35 In all families tested, mutational profiles from cffDNA haplotyping are consistent with

36 embryo biopsy profiles. Genome-wide fetal haplotypes are on average 97\% concordant with

37 the newborn haplotypes and embryo haplotypes.

\section{Conclusion}

39 We demonstrate that genome-wide targeted capture and sequencing of polymorphic SNPs

40 from maternal plasma cell free (cfDNA) allows haplotyping and copy number profiling of the 
41 fetal genome during pregnancy. The method enables the accurate reconstruction of the fetal

42 haplotypes and can be easily implemented in clinical practice.

\section{INTRODUCTION}

45 The discovery of cffDNA in the maternal plasma ${ }^{1}$ has spurred the development of non-

46 invasive prenatal genetic testing (NIPT). The advent of massively parallel sequencing

47 technologies enabled non-invasive screening for the most common fetal aneuploidies

48 (trisomy 21, 18 and 13) with high accuracy, which leverage rapid worldwide implementation

49 of NIPT-A in routine prenatal care ${ }^{2,3}$. NIPT-A became popular because it can be applied from

5010 weeks of pregnancy, reduces the risk of procedure-related miscarriage and technical

51 challenges associated with invasive prenatal testing. The accuracy largely outperforms the

52 traditional first and second trimester risk assessment tests. Its implementation resulted in a

53 significant drop of unnecessary invasive procedures ${ }^{4,5}$.

55 In addition to aneuploidy detection, monogenic diseases can be identified by cffDNA

56 analysis $^{6-8}$. Although the incidence of single-gene disorders is estimated to be about $1 \%$ of all

57 life births ${ }^{9}$ and over 7000 monogenic diseases are known ${ }^{10}$, non-invasive prenatal testing for

58 monogenic disorders has only been performed on a limited number of pregnancies for a small

59 panel of genes. Different methods have been developed ${ }^{11-15}$, but currently none has been

60 widely adopted in clinical practice. While the detection of paternally inherited alleles is

61 straightforward ${ }^{16}$, analysis of the maternally inherited allele has been hampered by the excess 
62 of maternal DNA in cfDNA. Analytical approaches that allow non-invasive diagnosis of

63 maternally inherited dominant or autosomal recessive monogenic diseases focus on the

64 determination of which allele the fetus has inherited. The relative mutation dosage (RMD) by

65 digital PCR measures the relative proportions of the mutant and wild-type alleles in the

66 maternal plasma ${ }^{11}$. However, a major disadvantage is that allele-specific probes are required

67 and, as a consequence, are only suitable for the detection of one single targeted variant per

68 test $^{17,18}$. Haplotype-based methods deduce the fetal genotype by measuring the relative counts

69 of alleles on haplotype blocks linked with the mutant allele and wild-type allele in the

70 maternal plasma ${ }^{19}$. Lo et al. ${ }^{13}$ and Kitzman et al. ${ }^{14}$ demonstrated that whole-genome sequencing of maternal plasma DNA to 65 -fold and 78-fold coverage allows the deduction of a genome-wide genetic and mutational profile of the fetus, opening opportunities to detect virtually all inherited monogenic diseases using one single platform. However, the high cost and the intensive computational analyses required for whole genome examinations currently prevent wide-scale clinical implementation. Target-based haplotyping methods are limited to tailored genes; a series of probes for the targeted capture of SNPs flanking a particular locus needs to be selected $a$ priori and require 200- to 1000-fold depth of cfDNA sequencing ${ }^{6,20,21}$. Hence, this approach requires disease specific work-up and cannot be universally applied for the generic diagnosis of monogenic disorders.

81 We previously developed a genome-wide single-cell haplotyping method, coined

82 haplarithmisis, which enables concurrent haplotype and copy number determination ${ }^{22}$. 

sites and to measure the copy of inherited parental haplotype. This method has been clinically implemented for comprehensive embryo pre-implantation genetic testing (PGT) for both monogenic disorders and aneuploidy ${ }^{23}$. Here, we tailor the approach for non-invasive prenatal haplotyping, and validated the method on families that underwent PGT-M where embryo haplotypes and newborn haplotypes of the uterine transferred embryos are available. We demonstrate the feasibility of cffDNA based haplotyping as a generic method for noninvasive prenatal detection of inherited monogenic diseases and aneuploidy detection.

\section{MATERIALS AND METHODS}

94

95

\section{Study design}

In total, nine families at risk for dominant or recessive disorders following PGT-M were included in this study (Table 1). The haplotype and mutational status of embryos were determined during routine PGT-M workflow at UZ Leuven hospital, and a healthy embryo was transferred. Genomic DNAs from the family members, including mother, father, and a close relative (either an affected offspring or parents of the couple) were collected. Maternal plasma cfDNA was later obtained from the pregnant woman following PGT-M and embryo transfer (Supplementary Materials and Methods). In three families, approval to sample the neonate was provided and DNA was obtained. In addition, one family with a trisomy 21 child was included to create spike-in DNA samples, simulating the fetal fraction observed in 
maternal plasma, and evaluate the performance of the method to detect aneuploidy. The workflow of this study is illustrated in Supplementary Figure S1.

107 This study was approved by the local Ethical Committees of the University Hospital Leuven 108 (S59324). Women with a successful pregnancy following PGT-M were recruited at the UZ 109 Leuven Hospital, with informed consent.

\section{DNA library preparation and targeted sequencing}

112 DNA libraries were prepared using the SureSelect ${ }^{\text {XTHS }}$ Target Enrichment System for

113 Illumina Paired-End Sequencing (Agilent Technologies, California, USA). Genomic DNAs

114 were processed according to manufacturer's recommendations. In the case of cfDNA

115 samples, between 5-20 ng were used for input, and the number of cycles for pre-hybridization

116 PCR was optimized to 11 to generate 500-1000 ng of DNA libraries. Unique molecular

117 identifiers (UMI) were added to DNA fragments before PCR amplification. End repair and A-

118 tailing, ligation and sample purification steps were performed following manufacturer's

119 instructions. DNA libraries were hybridized to a $45 \mathrm{Mb}$ custom capture library, which targets

120250,000 SNPs that includes $~ 250$ disease regions, subtelomeric and pericentromeric regions,

121 and sex chromosomes. The capture library was designed based on the HumanCytoSNP-12

122 BeadChip (Illumina, California, USA) using the Agilent SureSelect DNA Advanced Design

123 Wizard (https://earray.chem.agilent.com/suredesign/) with 2x tiling density, most stringent masking, and max performance boosting. Following hybridization and successful 
amplification, post-capture libraries were evaluated on Agilent 4200 Tapestation system

126 (Agilent Technologies) using High Sensitivity D1000 SCreeTape. Concentrations were also

127 measured by Qubit HS dsDNA Assay kit (ThermoFisher, Massachusetts, USA) before

128 pooling. Pools were clustered using an Illumina cBot and sequenced with paired-end 150

129 reads on an Illumina NextSeq500 in high output mode. Three newborns DNA were sequenced on an Illumina HiSeq4000 machine without UMI barcode.

\section{Sequencing alignment and variant calling}

133 Quality of the pair-end sequencing data was checked by FastQC v0.10.1 ${ }^{24}$. Sequencing reads were aligned to GRCh37 with decoy sequences included (hs37d5) by BWA-MEM v0.7.17 ${ }^{25}$ and UMI barcodes were transferred to bam file as RX tag. Duplicates were marked using Picard MarkDuplicates (the Broad Institute) with the awareness of UMI barcodes. Read pairs that mapped to the same genomic location and with identical molecular barcodes were grouped and ranked by base quality. Read pairs with the highest score from each molecular barcode families were kept, and PCR duplicates were removed. Low quality mapping reads

$140(<20)$ and secondary alignment were filtered for downstream analyses. We used Genome

141 Analysis Toolkit ${ }^{26}$ (GATK) software suite to perform variant calling. HaplotypeCaller was used to call variants from family gDNA samples jointly and parental genotypes were phased by PhaseByTransmission. Maternal plasma samples were handled separately, and allele counts were collected using ASEReadCounter by counting pair-end fragments requiring the overlapping bases to be identical, minimal mapping quality greater than 20 , and base quality 
146 greater than 2. Only sites with more than 30 total alleles count in maternal plasma were used

147 for analysis.

150 The principles of cffDNA haplotyping are presented in Figure 1. Briefly, by targeted

151 sequencing genomic regions genome-wide for family members and maternal plasma cfDNA,

152 genotypes and allele counts are determined for captured SNPs. Parental genotypes are phased

153 via an available genotype derived from a close relative, either an affected child or parents of

154 the couple as previously described ${ }^{22}$. The parental genotypes are divided into 5 groups based

155 on paternal and maternal allele combinations (Supplementary Materials and Methods). A

156 SNP locus is defined informative when the genotype of one parent is heterozygous and the

157 other is homozygous for this SNP. The informative SNPs are categorized as paternal or

158 maternal. An informative SNP is defined "paternal" when the father's genotype is

159 heterozygous, and the mother's genotype is homozygous. Similarly, an informative SNP is

160 defined "'maternal", when the mother's SNP genotype is heterozygous and the father's SNP

161 genotype is homozygous. These paternal and maternal informative SNP loci are then

162 subcategorized (P1, P2 or M1, M2) according to the informative phased parental SNP

163 genotypes (Figure 1a).

165 To deduce the fetal haplotype from cfDNA, we infer the alleles that originate from the fetus.

166 For the paternal SNP category, we can easily infer the paternally inherited alleles in the fetus 
167 that differ from the maternal background alleles present in the cfDNA (Figrue 1b). For the maternal SNP category, we cannot straightforwardly distinguish the maternally inherited allele of the fetus from cfDNA as both maternal alleles are present. Nevertheless, the maternal allele inherited by the fetus will be overrepresented in maternal plasma comparing

171 to the untransmitted allele. Thus, we based the haplotyping of the fetal genome on the determination of the fetal allele ratio (FAR) that measures the proportion of fetal allele in cfDNA. First, the fetal fraction (FF) is calculated by dividing the number of reads that exhibit a paternal specific allele by the total number of reads using Type 1 SNPs (Supplementary

Materials and Methods). Then the FAR values are measured for SNPs where either parent has a heterozygous SNP genotype. The FAR from consecutive informative SNPs is segmented for each SNP subcategory (P1, P2 or M1, M2) separately and then jointly interpreted, defining the haplotype blocks inherited from paternal $\mathrm{H} 1$ and $\mathrm{H} 2$ or maternal $\mathrm{H} 1$ and $\mathrm{H} 2$, and pinpointing homologous recombination sites between the parental $\mathrm{H} 1$ and $\mathrm{H} 2$

(Figure 1b). FF is used as a standard for segmented FAR value to determine homolog inheritance and to quantify copy number (Supplementary Materials and Methods).

\section{cffDNA haplotyping validation and performance}

To validate cffDNA haplotyping, we matched the cfDNA derive haplotypes to neonatal haplotypes and array-based single cell embryo haplotypes. Both mutational status and genome-wide haplotype concordance were compared and measured. To assess the performance of the cffDNA haplotyping under effects of different factors, downsampling and 

Methods.

\section{Aneuploidy detection}

Synthetic spike-in samples were made to simultaneously infer fetal haplotype and detect aneuploidy. Mixed samples were created by combining $20 \%$ and $10 \%$ of DNA from the affected proband with the respective pairs $80 \%$ and $90 \%$ of DNA from the mother.

Chromosomal abnormalities result in FAR value deviation from the expected FF. Statistical ttest was performed to measure shifting patterns. The haplotyping result and non-homologous disjunction from cfDNA were confirmed with the proband DNA (Supplementary Materials and Methods).

\section{RESULTS}

\section{Non-invasive prenatal testing for monogenic disorders}

202 Three families have an affected offspring, and six have parents of the couple available to

203 phase the parental genotypes (either paternal or maternal, depending who the carrier of

204 variant is). It is possible to infer both the paternal and maternal haplotype inheritance with an 205 affected offspring for phasing. Phasing with parents of either father or mother determines the 206 origin of the mutant allele in either the father or the mother (Supplementary Figure S2) and 207 only inheritance of the paternal or maternal haplotype is deduced. As a general observation 208 from raw FAR values of informative SNPs (Supplementary Figure S3), the maternal 
inheritance of homologous chromosome segments is more difficult to deduce visually due to

210 the overwhelming maternal DNA background. However, we enable haplotyping of the

211 maternally inherited genome of the fetus by FAR segmentation.

212

213 We verified the mutational profile derived from cffDNA haplotyping against the newborn

214 profile in the first instance. In three families (Family 1_181, 4_158 and 6_150), we

215 determined neonatal haplotypes following targeted sequencing. Family 1_181 presents an

216 autosomal recessive disorder, in which unaffected parents are heterozygous carriers for a

217 variant in the same gene (Table 1). Haplotyping of the cffDNA identifies the paternal and

218 maternal haplotype blocks linked with the wild-type allele at the locus of the PPT1 gene,

219 indicating that the fetus is not at risk for the disease. The haplotype obtained from bulk DNA

220 of the newborn child using conventional familial analysis, confirmed accurate haplotyping-

221 based NIPT-M and concordant positioning of homologous recombination sites (Figure 2a

222 and Supplementary Materials and Methods). For the other two families (Family 4_158 and

223 6_150), the disease is autosomal dominant, and the father carries the variant. Paternal parents

224 were used for phasing. The presence of a paternal haplotype block linked with the wild type

225 allele confirmed the transfer of unaffected embryo and is concordant with the newborn child

226 haplotype (Supplementary Figure S4a and b).

228 For all nine families, we validated the mutational profile determined from cfDNA to the

229 embryo biopsy haplotyping results from PGT SNP array-based haplarithmisis analyses. In all 
231 for Family 2_186, two autosomal recessive diseases were investigated in a single PGT. Using

232 cffDNA haplotyping, we ascertained the absence of paternal and maternal haplotype linked

233 with the mutant alleles for type 1 Gaucher disease. The cell-free fetal haplotype revealed the

234 same haplotype from embryo as being carrier of maternal variant for mitochondrial DNA

235 depletion syndrome 6 indication (Figure 2b). Family 3_085 presents with an X-linked

236 dominant disorder. Since the mother is the carrier of the variant in the family and the fetus is

237 male, only the maternal haplotype inheritance is displayed in Figure 2c.

\section{Genome-wide cffDNA haplotyping accuracy}

240 To evaluate the overall performance of the method, we determined the accuracy of genome-

241 wide cffDNA haplotyping by comparing the results to conventional haplotypes derived from

242 DNA analysis of neonatal blood when available and to single-cell haplotypes of the

243 transferred embryo, following PGT-M. The haplotype blocks derived from born children or

244 single-cells were considered as references and haplotype blocks derived from maternal

245 plasma DNA were matched to the reference (Supplementary Figure S5a). Comparing to

246 neonatal genotypes, paternal and maternal informative SNPs could be deduced with $95.17 \%$

247 and $65.84 \%$ accuracy respectively for a $9.5 \%$ FF sample, when the prediction is only based

248 on locus specific raw allele counts. With the use of haplotypes, the paternal and maternal

249 genotype inference accuracy increases to $99.7 \%$ and $95.64 \%$, respectively. Haplotyping

250 accuracy is reduced near homologous recombination sites (Supplementary Figure S5b-c). 
251 The comparison of cffDNA haplotypes to newborn haplotypes (Figure 3a and

252 Supplementary Figure S6a), and to embryo haplotypes (Figure 3b and Supplementary

253 Figure S6b) both showed an average of 99\% paternal and 95\% maternal haplotype concordance (Supplementary Table S1).

255

256 Above $98 \%$ accuracy was achieved for paternal haplotyping regardless of FF and sequencing 257 depth in all cases, while maternal haplotyping accuracy varied from $90 \%$ to $97 \%$ and is $\mathrm{FF}$ and sequence depth dependent. Reduced haplotype resolution could be observed near the recombination sites where the maternal haplotypes accuracy drops below $95 \%$ in a region of about $400 \mathrm{~Kb}$, whereas the paternal region of lower accuracy near crossovers ranges between 100 and 350Kb (Supplementary Table S1). Overall, haplotype accuracy and crossover resolution are impacted by FF, density of informative SNPs and sequencing depth (Supplementary Figure S5a). By simulating different fetal DNA fractions shown in cfDNA and maintaining the median coverage at a fixed 85-fold, the effect of FF on haplotyping accuracy and crossover was mapped. Paternal haplotypes were almost invariant to FF. In contrast, the accuracy of the maternally inherited haplotype as well as the resolution near

267 crossovers were both greatly affected by FF. While with $20 \%$ FF, the maternal haplotypes

268 were more than $98 \%$ accurate when compared with the reference haplotype at a homologous

269 recombination resolution of $200 \mathrm{~kb}$, the concordance decreased to less than $80 \%$ and a resolution of $1 \mathrm{Mb}$ with an FF of $5.5 \%$ (Figure 3c). 
272 However, it was possible to further improve the results when an affected offspring was used

273 for phasing. In such families, SNPs that are the heterozygous SNPs in both father and mother

274 (Type 4 SNPs) were applied to improve maternally inherited haplotypes. We converted such

275 ambiguous type 4 SNPs to unambiguous phased maternal SNPs after resolving paternal

276 haplotypes. By adding these extra SNPs to the inference of the maternally inherited haplotype

277 improved the accuracy when FF is low (Supplementary Figure S7). Though we anticipated

278 more accurate estimation of FARs and thus improved haplotyping accuracy by raising

279 sequencing depth especially when FF is low, we showed that even at low sequencing depth

280 robust fetal haplotypes could be obtained. To further investigate the effect of sequencing

281 depth on haplotyping, we downsampled two cfDNA-sequencing samples having $16.5 \%$ FF

282 and 9.5\% FF. Although the range of sequencing depth was 50-fold to 96-fold in our samples

283 (Supplementary Table S1), downsampling simulations showed that cfDNA haplotyping

284 performance was stably maintained at 40-fold sequencing depth for $16.5 \% \mathrm{FF}$ and was only

285 reduced to below 95\% concordance at 30-fold sequencing depth (Figure 3d). With FF at

$2869.5 \%$, haplotyping accuracy also only dropped greatly when sequencing depth reduced to

287 below 30\% of original depth (Supplementary Figure S8).

289 Aneuploidy detection using cffDNA haplotyping

290 In addition to inherited monogenic disease detection (NIPT-M), we explored the capability of

291 the methodology to detect simultaneously aneuploidy (NIPT-A) using synthetic spike-in

292 samples. As illustrated in Supplementary Figure S9, a trisomy would lead to a deviation of 
the segmented FAR value from the expected FF. In a maternally inherited trisomy, the segmented FAR value of the paternal SNPs shifts only marginally; however, maternal FAR values will shift systematically away from the FF value of the diploid autosomes. For instance, assuming $10 \% \mathrm{FF}$ and 100-fold coverage, we would expect that maternal FAR value to shift to $4.76 \%$ or $14.29 \%$ rather than $0 \%$ or $10 \%$. While for a paternally inherited trisomy, both paternal and maternal FAR values deviate from the expected FF (Supplementary Figure S9a and b). Accordingly, our data revealed that the paternal FAR values on chromosome 21 presented close to the expected normal FF levels, while the maternal FAR values for both M1 and M2 subcategories shifted away from the expected FF, being near to the theoretical trisomy FAR values. Using $t$-test, maternal (M1 and M2) mean FAR values of chromosome 21 showed significant difference from their corresponding subcategorical mean

304 FAR values of other chromosomes (Supplementary Figure S10). As a result, a maternally inherited trisomy 21 was determined (Figure 4a). The predicted haplotypes have both maternal haplotypes present, indicating that the trisomy is the consequence of a maternal

307 meiotic nondisjunction. Sequencing data from the proband also confirmed this and showed a 308 concordant phasing between the spike-in samples and the child DNA (Figure 4b). Despite 309 trisomy 21, the genome-wide haplotyping demonstrated $99.9 \%$ and $99 \%$ accuracy for 310 paternal and maternal inheritance for $20 \%$ FF spike-in sample, and $99.8 \%$ and $97 \%$ for $10 \%$ 311 FF spike-in sample. 
314 We demonstrate that genome-wide targeted capture and sequencing of polymorphic SNPs

315 from maternal cfDNA along with parental, additional family member's DNA, allows

316 haplotyping and copy number profiling of the fetal genome during pregnancy. cffDNA

317 haplarithmisis analysis enables the accurate reconstruction of the fetal haplotypes without the

318 need for deep sequencing nor whole genome sequencing analyses. A wide spectrum of

319 monogenic disorders and aneuploidy are readily detectable via this approach. This opens the

320 venue for concurrent NIPT-M and NIPT-A. With uptake of the testing and technology

321 refinement, detection of sub-chromosomal aneuploidy and copy number detection will

322 become feasible as well.

323

324 We envision cffDNA haplarithmisis to be a universal NIPT that avoids the necessity to design

325 specific panels defining particular loci to be analyzed. Cost is one of the major factors that

326 limits the scalability of NIPT-M. The capture design and targeted sequencing used in this

327 method can make NIPT-M more affordable in the long term. In contrast to the RHDO method

328 where more than 200-fold coverage of the target loci is required, the method leverages

329 segmentation of fetal allele ratio over multiple informative SNPs that allows a significant

330 reduction of the required sequencing depth. Samples can be multiplexed to further reduce

331 costs. In contrast to current whole genome cffDNA haplotyping methods that require whole

332 genome sequencing to achieve genome-wide fetal haplotyping resolution, targeted-

333 sequencing reduces the amount of sequencing needed and reduces the complexity of

334 processing deep whole genome data. Targeted sequencing and haplotyping of a genome wide 
336 the haplotype inference accuracy, unique molecular identifiers were incorporated to reduce

337 amplification artifacts and technical biases were removed by using multiple filtering criteria,

338 monitoring sequencing errors and applying dynamic bias corrections. We set standard

339 classification rules based on FF to assure sufficient evidences supporting the homolog

340 assignment (Supplementary Materials and Methods). A range of conditions, including

341 dominant, recessive and X-linked monogenic diseases can be assessed in this generic non-

342 invasive prenatal diagnosis test. Multiple variants can be identified in one test without

343 variant-specific designs, as shown in the case of Family 2. In case of aneuploidies, the

344 parental origin and the segregation error (meiotic or mitotic) can be deduced.

346 Parental haplotypes are deduced from the genotypes of other family members.

347 Sometimes, those relatives are not available. Direct parental haplotyping through long-read ${ }^{20}$

348 or linked-read ${ }^{6,27}$ technology can offer a solution to haplotype inference of the family without

349 additional family members. In the longer haul, the availability of population haplotypes will

350 allow inference of the disease allele, especially for the most common recessive disorders ${ }^{28,29}$.

351 Those haplotypes could be imputed to reduce the need for parental and grandparental

352 haplotyping. Also, although the method was designed to be generically applicable to

353 monogenic diseases detection, it is not suitable for de novo variant detection. To overeome-

354 this, amplicon sequencing of specific loci has been explored. Zhang et al. ${ }^{30}$ demonstrated a 
356 paternally inherited disease-causing variants. It might become possible to add capture probes

357 to the current design. However, the approach should be compensating for the high sequencing

358 depth required for de novo mutation detection as compared to the relative lower sequencing

359 depth required here. A more general limitation of the method is that meiotic homologous-

360 recombinations occurring near the mutant gene would not allow to infer whether the fetus is

361 earrier of variant or not. We reached an overall 97\% concordance with embryo and neonatal

362 haplotypes and the discordance arises mainly from the homologous recombination regions.

363 As a general limitation, meiotic homologous recombinations occurring near the mutant gene

364 would not allow inference of whether the fetus is a carrier of variant or not. In such case, an

365 invasive test can be recommended. The accuracy of the homologous recombination and

366 haplotype construction are determined by the interplay of the fetal fraction, the density of

367 informative SNPs and the sequencing depth around the genomic region. Low fetal fraction

368 leads to reduced accuracy particular for the inference of the maternally inherited haplotype,

369 but this may be remedied by higher density of informative SNPs. Though we yielded

370 conclusive results for all clinical cases presented in the study, from simulation we estimated

371 that to get an overall accuracy in maternally inheritance haplotyping above $90 \%$ requires

$3727.8 \%$ fetal fraction with moderate sequencing depth. Paternally inherited haplotypes can

373 readily be detected even when the fetal fraction is $3 \%$ at about 85 -fold coverage

374 (Supplementary Figure S11). Of note, we have one case where the mother is a carrier of

$375 \sim 1.5 \mathrm{Mb}$ duplication causing Charcot-Marie-Tooth disease type 1 syndrome. Though

376 maternal copy number variations may interfere the FAR estimations, the method showed 
377 tolerance for the excessive maternal allele background for relatively small duplication. As we

378 can already detect the maternal copy number in this case (Supplementary FigureS12a-b),

379 parental copy number of larger sizes are very likely be detected, and the region can be taken

380 into consideration for proper interpretation of results. Placental chromosomal mosaicism

381 might be another factor affecting the analysis. From PGT-A of blastocysts it is becoming

382 clear that many blastocysts carry aneuploidies in a fraction of the cells. Although we

383 demonstrated that the impact of embryonic aneuploidy seems to be marginal during prenatal

384 development ${ }^{31}$, placental mosaic aneuploidies have been reported in NIPT ${ }^{32,33}$. It remains to

385 be determined whether mosaic aneuploidies would interfere with this approach.

386

387 Generic methods to haplotype and profile aneuploidies in embryos have transformed

388 preimplantation genetic testing for monogenic diseases (PGT-M) and are becoming an

389 integral aspect of in vitro fertilization procedures ${ }^{34,35}$. Because of the risk for a spontaneous

390 pregnancy during the PGT procedure and possible laboratory procedure errors ${ }^{36}$, a prenatal

391 diagnostic test (chorionic villus sampling or amniocentesis) is currently highly recommended

392 to confirm the transfer of an unaffected embryo. Since fetal genetic testing for monogenic

393 disorders currently requires an invasive procedure which is estimated to have a $1 \%$ risk for

394 procedure induced miscarriage, may have miscarriage risk $^{37}$, most families renounce from

395 undergoing the test. cffDNA haplarithmisis represents a safer alternative for these families.

396 As proof-of-concept, we actually performed targeted sequencing on families that underwent

397 PGT-M by genome-wide haplotyping and do demonstrate a very high concordance of the 
embryo single-cell and cfDNA derived haplotypes. In families that undergo PGT-M by

399 haplotyping, NIPT-M can be streamlined as one workflow where phasing of parental

400 genotypes has already been performed in the PGT-M process. Hence, non-invasive prenatal

401 fetal haplotyping would require only analysis of the targeted cfDNA.

402

403 Genetic carrier screening has been offered to individuals and couples based on family history

404 or ethnic background. Screening of cystic fibrosis and thalassemia have been recommended

405 and is rolled out for general preconception and prenatal populations ${ }^{38}$. Moreover, technology

406 advances in next generation sequencing and better understanding of disease causing variants

407 continuously drive expansion of screening panels ${ }^{39}$. As an increasing number of genetic

408 disorders recognized to be practical for screening, the raising awareness that each individual

409 can be a carrier of variants that may cause recessive disorders and the increasing uptake of

410 carrier screening in the general population ${ }^{40}$, new approaches to reduce the transmission of

411 disease alleles that leads to severe morbidity and mortality are desirable. In such scenario, our

412 method could be applied in combination with carrier screening program to help couples at

413 high risk for inherited diseases who are at high risk for inherited diseases but cannot, or do

414 not want to or do not have access to preimplantation genetic testing to make autonomous

415 reproductive decisions.

417 In summary, haplarithmisis makes non-invasive genome-wide fetal haplotyping and

418 aneuploidy detection with targeted sequencing accessible to all. This universal cffDNA 
419 haplotyping approach could easily be adopted by genetic testing laboratories and would

420 provide comfort to both the couples and the care-takers involved. Following this proof-of-

421 concept study, we expect expanded clinical studies to further validate the method more

422 precisely.

423

\section{Data availability}

425 All sequencing data from 9 families underwent PGT and 1 family with trisomy 21 proband

426 have been submitted to the European Genome-phenome Archive (https://www.ebi.ac.uk/ega/)

427 under accession numbers EGAS00001003634.

428

429 Code availability

430 Code is available in GitHub at https://github.com/vermeeschlab/cfDNAhaplotyping

431

432 Acknowledgements

433 We are grateful to all families that participated in this study. This work was funded by Agilent, 434 by KULeuven funding (C1/018), the Horizon 2020 WIDENLIFE: 692065 to J.R.V. and T.V 435 and by the Fonds voor Wetenschappelijk Onderzoek (FWO-Vlaanderen: G080217N) to J.V.

436 D.V. was supported by FAPESP (2017/23448-8).

438 Disclosure

439 The authors declare no conflicts of interest. 
441 1. Lo, Y. M. D. et al. Presence of fetal DNA in maternal plasma and serum. The Lancet 350, 485-487 (1997).

2. Bayindir, B. et al. Noninvasive prenatal testing using a novel analysis pipeline to screen for

444 all autosomal fetal aneuploidies improves pregnancy management. Eur. J. Hum. Genet. 23, 1286-1293 (2015).

3. Bianchi, D. W. \& Chiu, R. W. K. Sequencing of Circulating Cell-free DNA during Pregnancy. N. Engl. J. Med. 379, 464-473 (2018).

4. Song, K., Musci, T. J. \& Caughey, A. B. Clinical utility and cost of non-invasive prenatal testing with cfDNA analysis in high-risk women based on a US population. J. Matern. Fetal Neonatal Med. 26, 1180-1185 (2013).

451

5. Hui, L., Hutchinson, B., Poulton, A. \& Halliday, J. Population-based impact of noninvasive prenatal screening on screening and diagnostic testing for fetal aneuploidy. Genet. Med. 19, $1338-1345$ (2017).

454

6. Hui, W. W. I. et al. Universal Haplotype-Based Noninvasive Prenatal Testing for Single Gene Diseases. Clin. Chem. 63, 513-524 (2017).

7. New, M. I. et al. Noninvasive Prenatal Diagnosis of Congenital Adrenal Hyperplasia Using (2014).

8. Xu, Y. et al. Haplotype-based approach for noninvasive prenatal tests of Duchenne muscular dystrophy using cell-free fetal DNA in maternal plasma. Genet. Med. 17, 889-896 (2015). 
461 9. Fujikura, K. Global Carrier Rates of Rare Inherited Disorders Using Population Exome 462 Sequences. PLOS ONE 11, e0155552 (2016).

463 10. Bell, C. J. et al. Carrier Testing for Severe Childhood Recessive Diseases by Next464 Generation Sequencing. Sci. Transl. Med. 3, 65ra4-65ra4 (2011).

465 11. Lun, F. M. F. et al. Noninvasive prenatal diagnosis of monogenic diseases by digital size 466 selection and relative mutation dosage on DNA in maternal plasma. Proc. Natl. Acad. Sci. $467 \quad \mathbf{1 0 5}, 19920-19925$ (2008).

468 12. Chiu, R. W. K., Cantor, C. R. \& Lo, Y. M. D. Non-invasive prenatal diagnosis by single 469 molecule counting technologies. Trends Genet. 25, 324-331 (2009).

470 13. Lo, Y. M. D. et al. Maternal Plasma DNA Sequencing Reveals the Genome-Wide Genetic 471 and Mutational Profile of the Fetus. Sci. Transl. Med. 2, 61ra91-61ra91 (2010).

472 14. Kitzman, J. O. et al. Noninvasive Whole-Genome Sequencing of a Human Fetus. Sci. 473 Transl. Med. 4, 137ra76-137ra76 (2012).

474 15. Fan, H. C. et al. Non-invasive prenatal measurement of the fetal genome. Nature 487, 320$475 \quad 324(2012)$.

476 16. Verhoef, T. I. et al. Non-invasive prenatal diagnosis (NIPD) for single gene disorders: cost 477 analysis of NIPD and invasive testing pathways. Prenat. Diagn. 36, 636-642 (2016).

478 17. Tsui, N. B. Y. et al. Noninvasive prenatal diagnosis of hemophilia by microfluidics digital $479 \quad$ PCR analysis of maternal plasma DNA. Blood 117, 3684-3691 (2011).

480 18. Barrett, A. N., McDonnell, T. C. R., Chan, K. C. A. \& Chitty, L. S. Digital PCR Analysis 481 of Maternal Plasma for Noninvasive Detection of Sickle Cell Anemia. Clin. Chem. 58, 
19. Chiu, E. K. L., Hui, W. W. I. \& Chiu, R. W. K. cfDNA screening and diagnosis of monogenic disorders - where are we heading? Prenat. Diagn. 38, 52-58 (2018).

20. Vermeulen, C. et al. Sensitive Monogenic Noninvasive Prenatal Diagnosis by Targeted Haplotyping. Am. J. Hum. Genet. 101, 326-339 (2017).

21. Parks, M. et al. Non-invasive prenatal diagnosis of spinal muscular atrophy by relative haplotype dosage. Eur. J. Hum. Genet. 25, 416-422 (2017).

22. Zamani Esteki, M. et al. Concurrent Whole-Genome Haplotyping and Copy-Number Profiling of Single Cells. Am. J. Hum. Genet. 96, 894-912 (2015).

23. Dimitriadou, E. et al. Principles guiding embryo selection following genome-wide haplotyping of preimplantation embryos. Hum. Reprod. 32, 687-697 (2017).

24. Andrews, S. FastQC: a quality control tool for high throughput sequence data.

25. Li, H. Aligning sequence reads, clone sequences and assembly contigs with BWA-MEM. ArXiv13033997 Q-Bio (2013).

26. McKenna, A. et al. The Genome Analysis Toolkit: A MapReduce framework for analyzing 497 next-generation DNA sequencing data. Genome Res. 20, 1297-1303 (2010).

27. Zheng, G. X. Y. et al. Haplotyping germline and cancer genomes with high-throughput linked-read sequencing. Nat. Biotechnol. 34, 303-311 (2016).

500 28. Browning, S. R. \& Browning, B. L. Haplotype phasing: existing methods and new 501 developments. Nat. Rev. Genet. 12, 703-714 (2011).

502 29. Zeevi, D. A. et al. Proof-of-principle rapid noninvasive prenatal diagnosis of autosomal 
30. Zhang, J. et al. Non-invasive prenatal sequencing for multiple Mendelian monogenic disorders using circulating cell-free fetal DNA. Nat. Med. 1 (2019) doi:10.1038/s41591018-0334-X.

31. Zamani Esteki, M. et al. In vitro fertilization does not increase the incidence of de novo copy number alterations in fetal and placental lineages. Nat. Med. 25, 1699-1705 (2019).

32. Brison, N. et al. Predicting fetoplacental chromosomal mosaicism during non-invasive prenatal testing. Prenat. Diagn. 38, 258-266 (2018).

33. van der Meij, K. R. M. et al. TRIDENT-2: National Implementation of Genome-Wide Non-Invasive Prenatal Testing as a First-Tier Screening Test in the Netherlands. Am. J. Hum. Genet. (2019) doi:10.1016/j.ajhg.2019.10.005.

34. Vermeesch, J. R., Voet, T. \& Devriendt, K. Prenatal and pre-implantation genetic diagnosis. Nat. Rev. Genet. 17, 643-656 (2016).

35. Handyside, A. H. 'Designer babies' almost thirty years on. Reproduction 156, F75-F79 (2018).

518 36. Wilton, L., Thornhill, A., Traeger-Synodinos, J., Sermon, K. D. \& Harper, J. C. The causes 519 of misdiagnosis and adverse outcomes in PGD. Hum. Reprod. 24, 1221-1228 (2009).

520 37. Salomon, L. J., Sotiriadis, A., Wulff, C. B., Odibo, A. \& Akolekar, R. Risk of miscarriage 521 following amniocentesis or chorionic villus sampling: systematic review of literature and updated meta-analysis. Ultrasound Obstet. Gynecol. 54, 442-451 (2019).

523 38. Bajaj, K. \& Gross, S. J. Carrier Screening: Past, Present, and Future. J. Clin. Med. 3, 1033- 
5241042 (2014).

525 39. Lazarin, G. A. \& Haque, I. S. Expanded carrier screening: A review of early 526 implementation and literature. Semin. Perinatol. 40, 29-34 (2016).

527 40. Haque, I. S. et al. Modeled Fetal Risk of Genetic Diseases Identified by Expanded Carrier 528 Screening. JAMA 316, 734-742 (2016).

529 
532 Figure 1. Principles of cffDNA haplotyping. a, Example of a family with an autosomal

533 dominant disorder. DNA from the parents and the affected offspring is first genotyped and

534 on the basis of the affected child's genotype, parental SNPs can be phased to determine the

535 transmission of paternal and maternal homologues, including the mutant allele. Paternal

536 informative SNPs, defined as heterozygous in the father and homozygous in the mother,

537 are identified as a step 1 phasing rule. The paternal homologue that is transmitted to the

538 affected child must contain the causative variant and is denoted homologue 1 (H1),

539 whereas paternal H2 carries the normal allele. Subsequently (step 2), informative SNPs are

540 categorized to define parental SNPs subcategories - P1 and P2 for paternal SNPs, and M1

541 and M2 for maternal SNPs. b, Determination of fetal haplotype inheritance was based on

542 fetal allele ratio (FAR) metric. Red and blue indicate paternal P1 and P2 SNPs

543 subcategories, and the same color code is also applied to distinguish M1 and M2 SNPs

544 subcategories. Segmentation on FAR values (step 3) was performed to define the haplotype

545 blocks derived from paternal $\mathrm{H} 1$ and $\mathrm{H} 2$ or maternal $\mathrm{H} 1$ and $\mathrm{H} 2$, thus indicating homologous

546 recombination sites between the parental $\mathrm{H} 1$ and $\mathrm{H} 2$.

547

548 Figure 2. cffDNA haplotyping analysis for monogenic disorders. Disease locus associated

549 chromosome cffDNA haplotyping result. For each sub figure, family pedigree information is

550 displayed in together with haplotyping. The reference haplotypes from either the born child

551 and/or from the embryo blastomere and cffDNA haplotyping results are shown. Color blue in 
552 haplotype plots indicates paternal haplotype inheritance and color red indicates maternal

553 haplotype inheritance. For cffDNA haplotyping results, both segmented FAR values and

554 derived haplotype blocks are shown. In segmented FAR values track, the red dotted line

555 represents segmented P1 or M1 FAR and blue for segmented P2 or M2 FAR, and the distance

556 between P1 and P2 or M1 and M2 segmentation in the same genomic region indicates fetal

557 fraction. We flipped FAR values of P1 subcategory around 0 and FAR values of M1 were

558 subjected to less than or equal to 0 in visualization for clear separation between informative

559 SNPs subcategories. Disease loci are indicated with a yellow vertical line. a, cffDNA

560 haplotype compared to neonate haplotype and embryo haplotype. The paternal homologue

561 carrying the variant is represented in dark blue and maternal homologue carrying the variant

562 is represented in dark red. The disease locus resided in light color block of both paternal and

563 maternal haplotypes, indicating wild-type alleles were transmitted. b-c, cffDNA haplotyping

564 results compared to the embryo haplotype. b. Two disease indications of the family are

565 shown. c, Inheritance of an X-linked disorder is shown.

566

567 Figure 3. Genome-wide cffDNA haplotyping accuracy. a, Illustration of density of SNPS,

568 sequencing depth and FF effects on haplotype accuracy and crossover resolution metrics. $\mathbf{b}$,

569 For a 9.5\%FF cfDNA samples, homologous recombination accuracy measured in 10Kb bins-

570 that flank out from both sides of true (neonatal) crossevers, is shown. On x axis, $\theta$ denotes

571 the true crossover breakpoint sites. Positive and negative distances correspend to extension to

572 the right and to the left side of breakpoints. a, Genome-wide comparison of cffDNA 
573 haplotyping results to the neonate haplotype from Family 1_181. For each chromosome, dark

574 and light blue represents paternal haplotyping and dark and light red represents maternal

575 haplotyping; the upper haplotype track refers to born child haplotype and lower track

576 represents cfDNA-based fetal halplotype. b, Genome-wide comparison of cffDNA

577 haplotypes to the embryo blastomere haplotype from Family 3_085. The upper track shows

578 the single-cell haplotype and lower track represents cfDNA derived haplotype. c, Simulation

579 of the impact of fetal fraction (FF) on haplotyping accuracy and resolution. d, Effect of

580 sequencing depth on the performance of cffDNA haplotyping for $16.5 \% \mathrm{FF}$.

581

582 Figure 4. Aneuploidy detection using spike-in samples. a, Maternally inherited trisomy 21

583 detected in spike-in DNA samples with $20 \%$ and $10 \%$ fetal fraction, respectively. In $20 \%$

584 spike-in sample, maternal FAR values dropped around $10 \%$ and $-10 \%$, indicating an extra

585 copy from the 'fetus' on chromosome 21 . Similarly, in the $10 \%$ FF, maternal FAR values

586 were around $5 \%$ and $-5 \%$. b, Validation of maternally inherited trisomy case using

587 sequencing data from the proband. The copy number plot (log2 ratio) indicated chromosomal

588 copy number on chromosome 21 , with black dot as $\log 2$ ratio of each target and red line as

589 segmented value. The paternal and maternal reference allele ratio from chromosome 21 also

590 deviates from standard reference allele ratio of 0.5 and the homologous recombinations are

591 consistent with spike-in samples derived result.

592

593 
Conflict of Interest Statement

\section{Conflict of Interest}

The authors have nothing to disclose. 
Figure 1

a

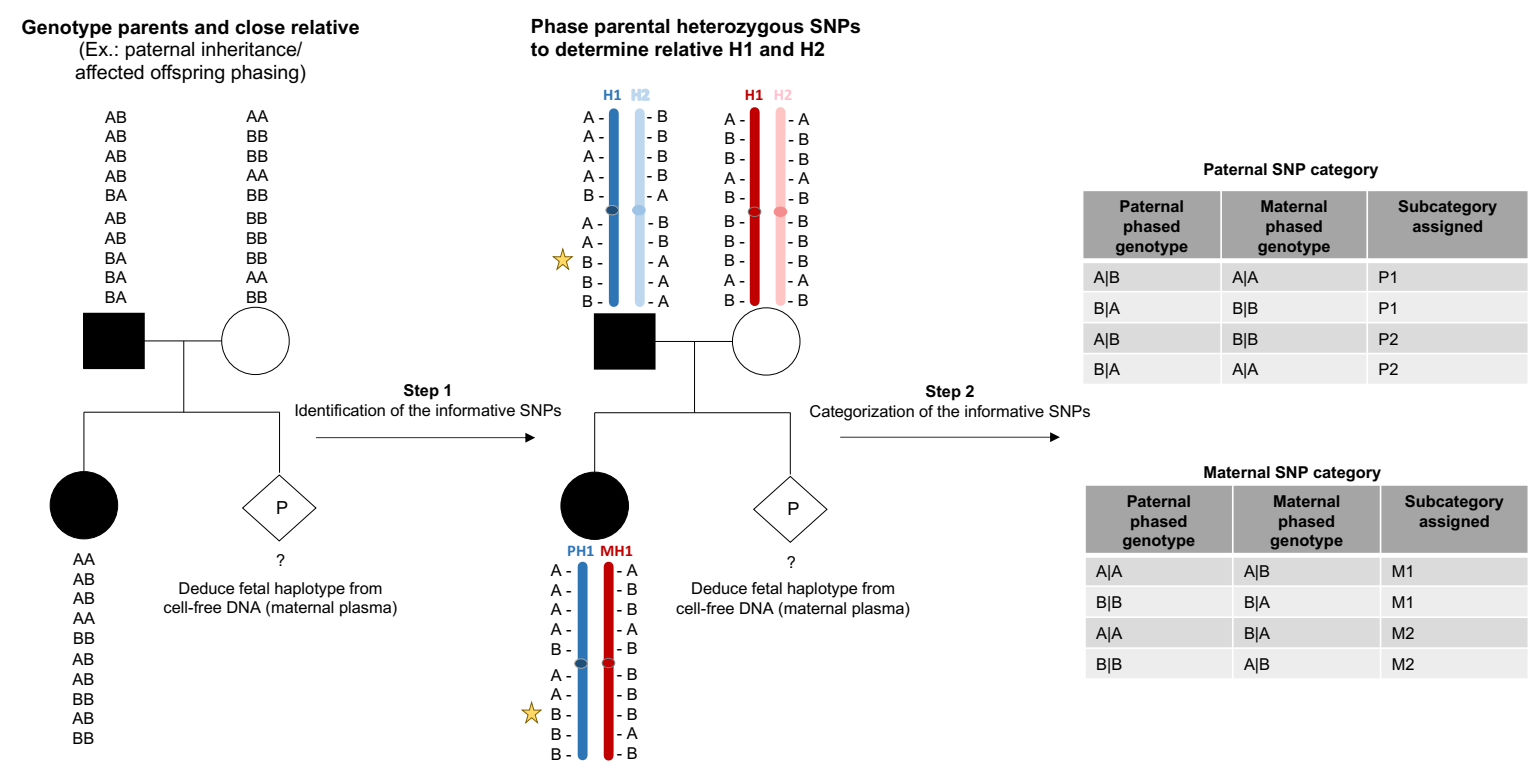

b

Determine fetal haplotype from cell-free DNA - Paternal SNP category
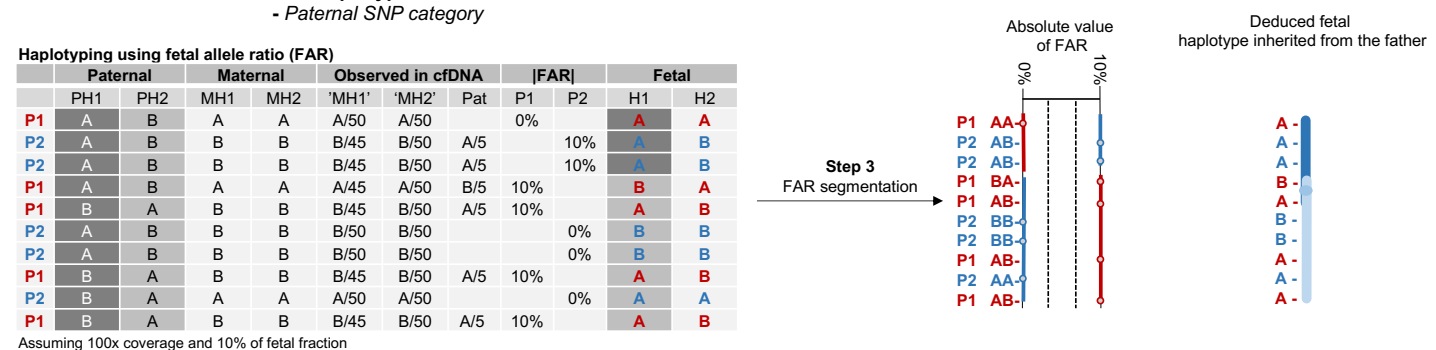

Determine fetal haplotype from cell-free DNA Maternal SNP category
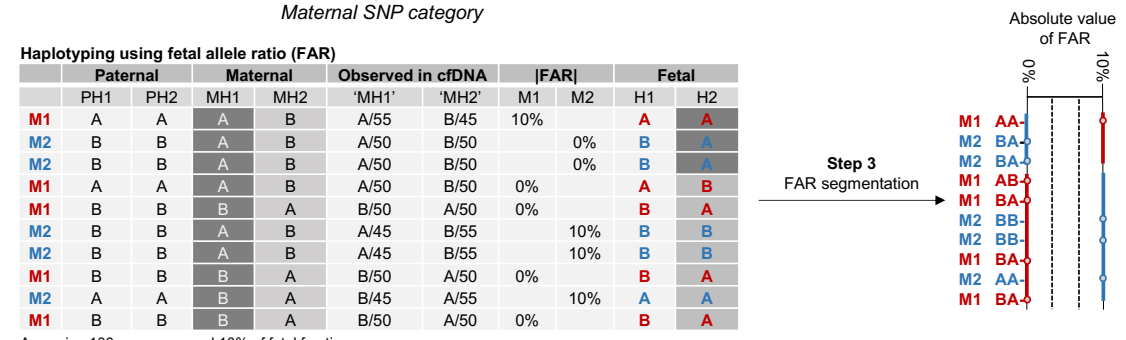

Deduced fetal

haplotype inherited from the mother

Haplotyping using fetal allele ratio (FAR)

\begin{tabular}{|c|c|c|c|c|c|c|c|c|c|c|}
\hline & \multicolumn{2}{|c|}{ Paternal } & \multicolumn{2}{|c|}{ Maternal } & \multicolumn{2}{|c|}{ Observed in cfDNA } & \multicolumn{2}{|c|}{ |FAR| } & \multicolumn{2}{|c|}{ Fetal } \\
\hline & $\mathrm{PH} 1$ & PH2 & MH1 & MH2 & 'MH1' & 'MH2' & M1 & M2 & $\mathrm{H} 1$ & $\mathrm{H} 2$ \\
\hline M1 & A & A & A & B & $\mathrm{A} / 55$ & $\mathrm{~B} / 45$ & $10 \%$ & & A & A \\
\hline M2 & B & B & $\mathrm{A}$ & B & A/50 & B/50 & & $0 \%$ & B & $\mathrm{A}$ \\
\hline M2 & B & B & A & B & $\mathrm{A} / 50$ & $\mathrm{~B} / 50$ & & $0 \%$ & B & $\mathrm{A}$ \\
\hline M1 & A & A & $A$ & B & $\mathrm{A} / 50$ & $\mathrm{~B} / 50$ & $0 \%$ & & A & B \\
\hline M1 & B & B & $B$ & A & $B / 50$ & $\mathrm{~A} / 50$ & $0 \%$ & & B & A \\
\hline M2 & B & B & $\mathrm{A}$ & B & A/45 & $\mathrm{B} / 55$ & & $10 \%$ & B & B \\
\hline M2 & B & B & $\mathrm{A}$ & B & $\mathrm{A} / 45$ & $\mathrm{~B} / 55$ & & $10 \%$ & B & B \\
\hline M1 & B & B & $\mathrm{B}$ & A & $B / 50$ & $\mathrm{~A} / 50$ & $0 \%$ & & B & A \\
\hline M2 & A & A & $B$ & A & $B / 45$ & $\mathrm{~A} / 55$ & & $10 \%$ & A & A \\
\hline M1 & B & B & B & A & $B / 50$ & $\mathrm{~A} / 50$ & $0 \%$ & & B & A \\
\hline
\end{tabular}

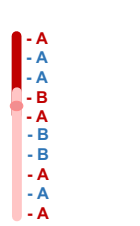


Figure 2

a

Family1_181

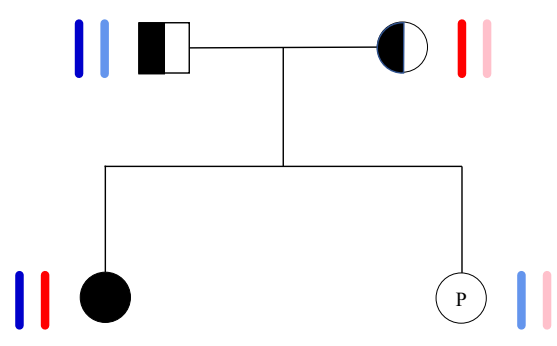

Family1_181

Chromosome 1

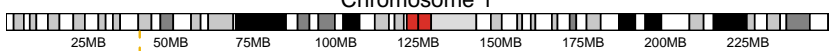

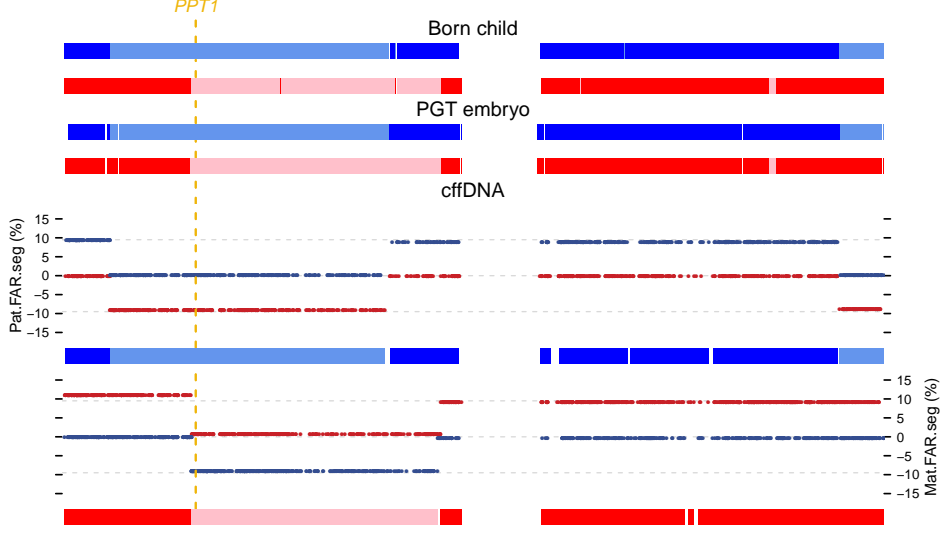

b

Family2_186



Family2_186

Chromosome 1

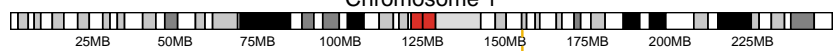

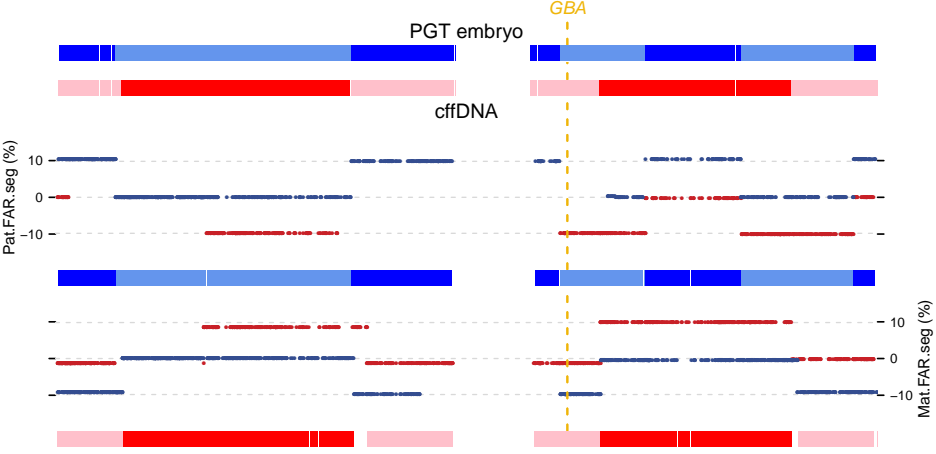

Family2_186

Chromosome 2

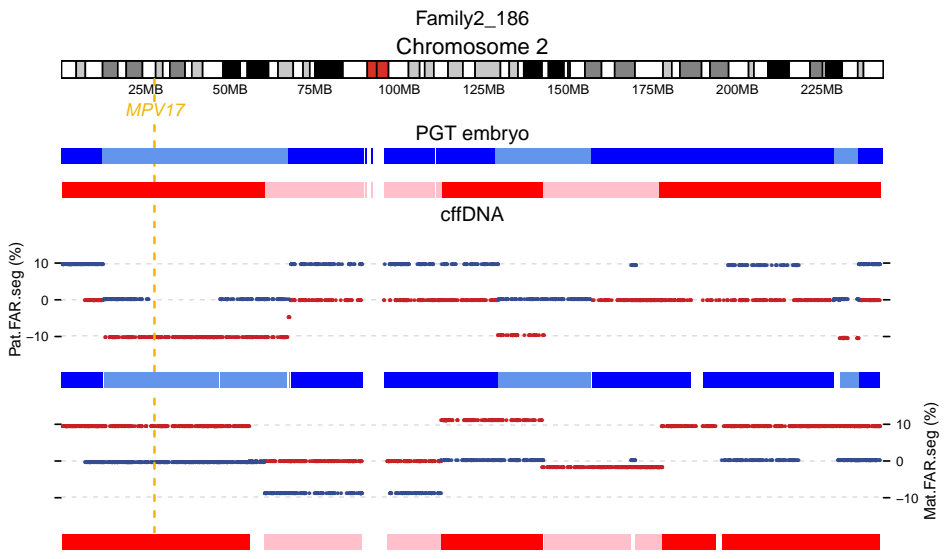

C

Family3_085

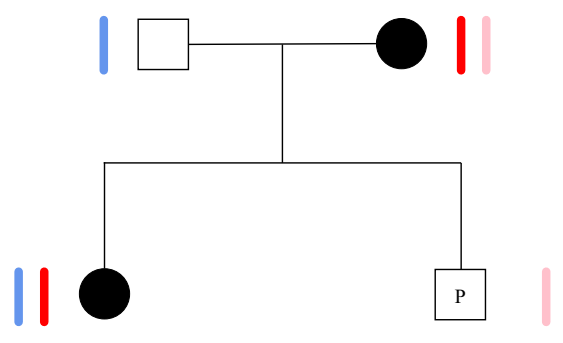

Family3_085

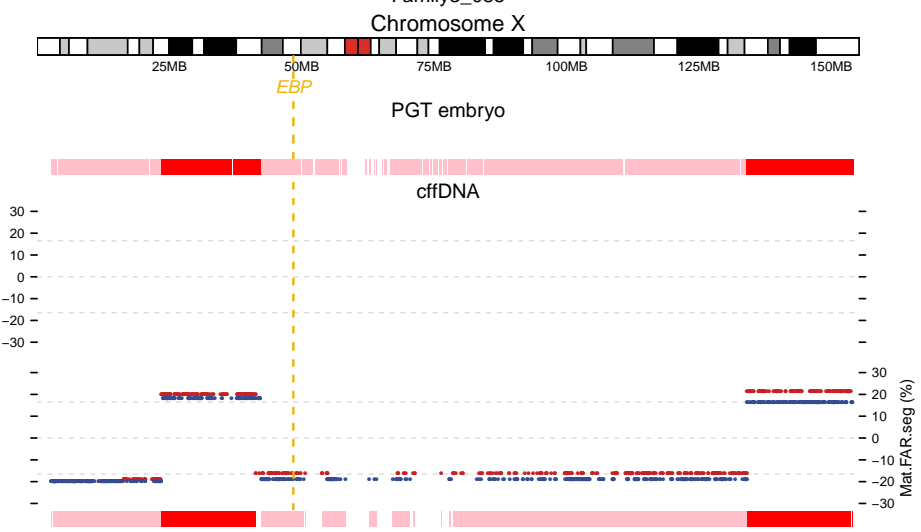




\section{Figure 3}
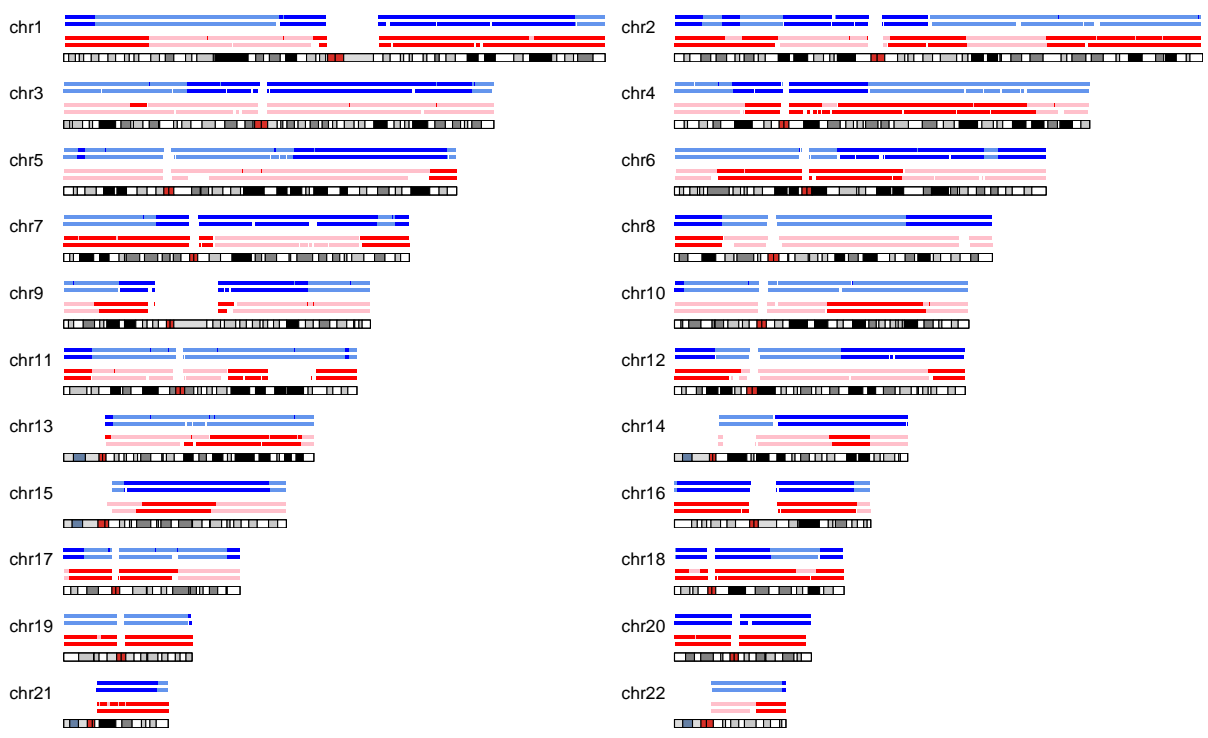

chr3 $\overline{=}=$

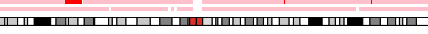

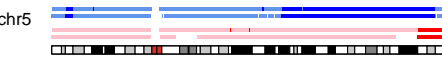

$7 \overline{\overline{\overline{2}}} \overline{\overline{2}}=$

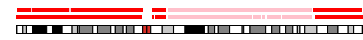

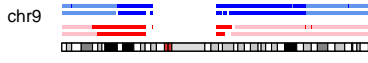

$\operatorname{chr11} \overline{\equiv=}$

chr13

ए110

chr15

$\operatorname{chr} 17=$

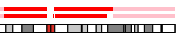

chr19 $\overline{\overline{\bar{\nu}}}$

chr21 $\overline{\overline{=}}$

要

$\operatorname{chr} X$

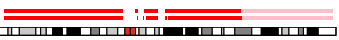

b

Family3_085_PGTembryo
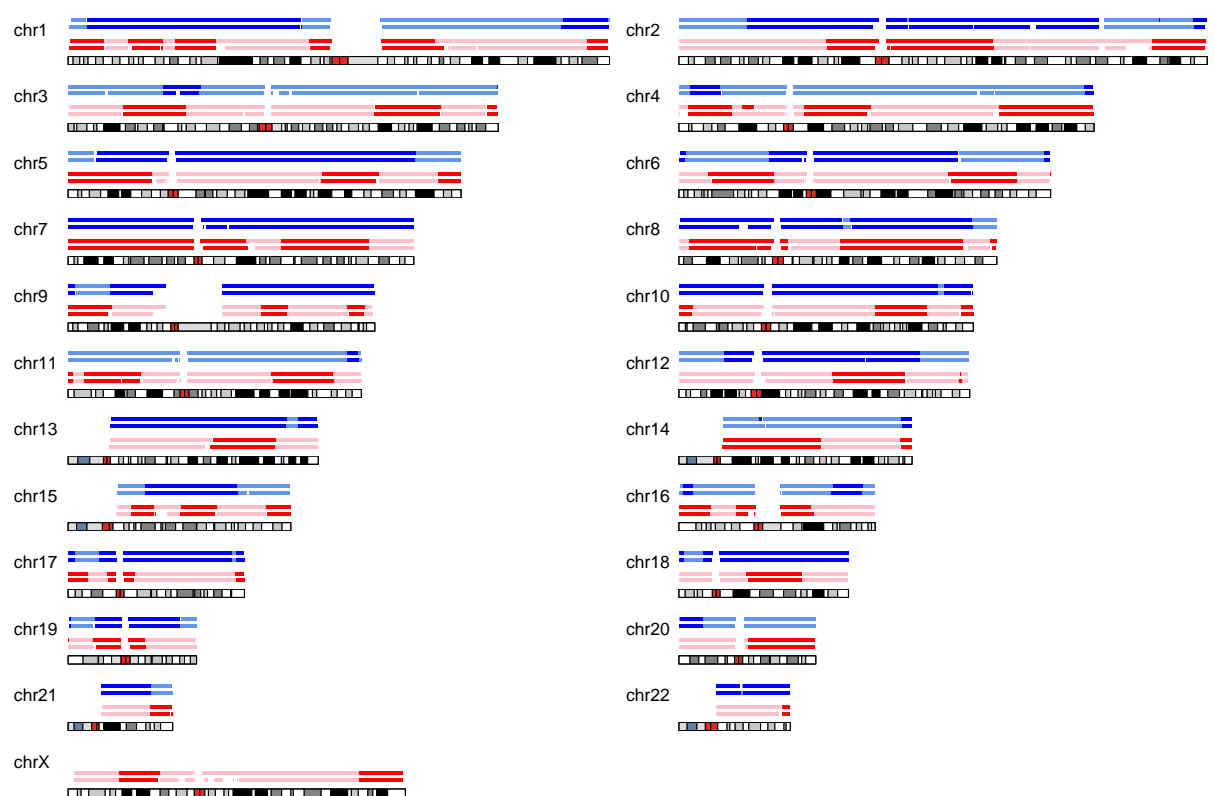

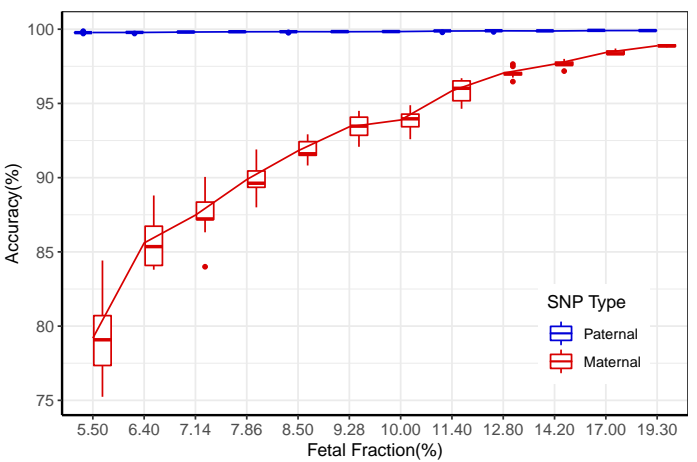

d

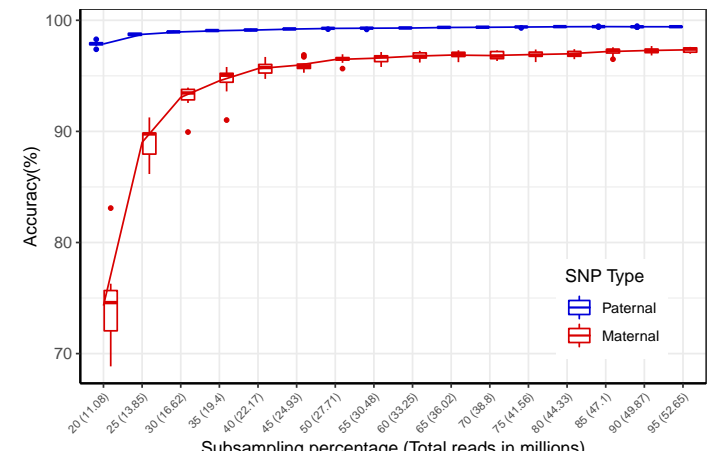

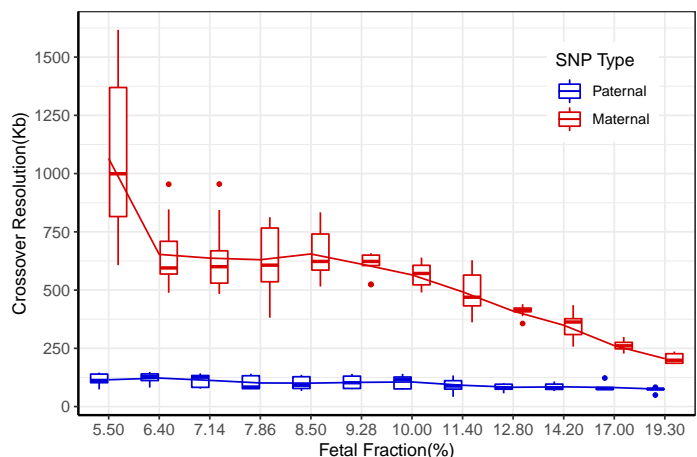

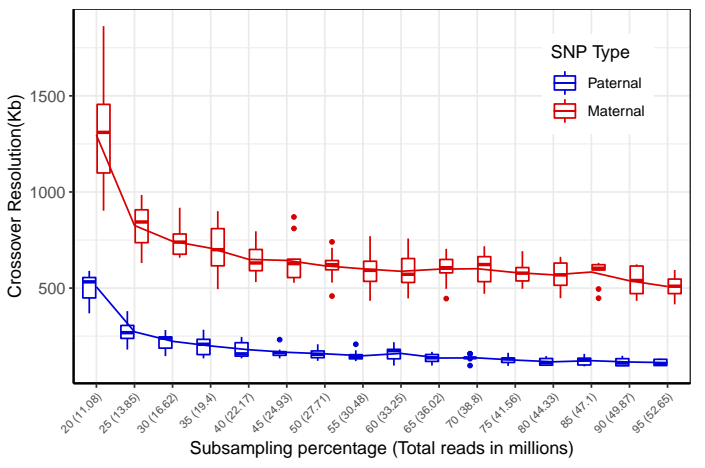


Figure 4

a

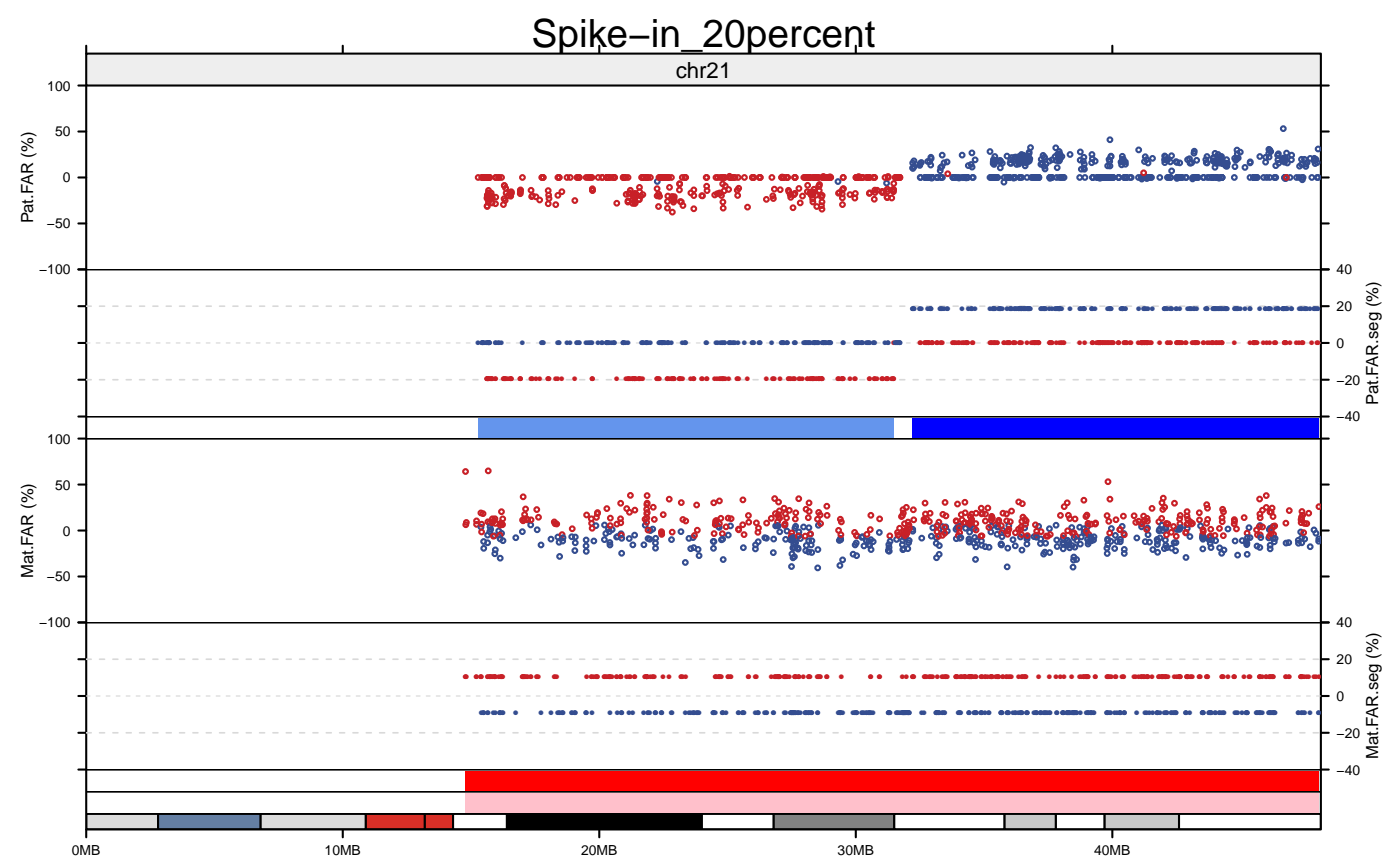

Spike-in_10percent

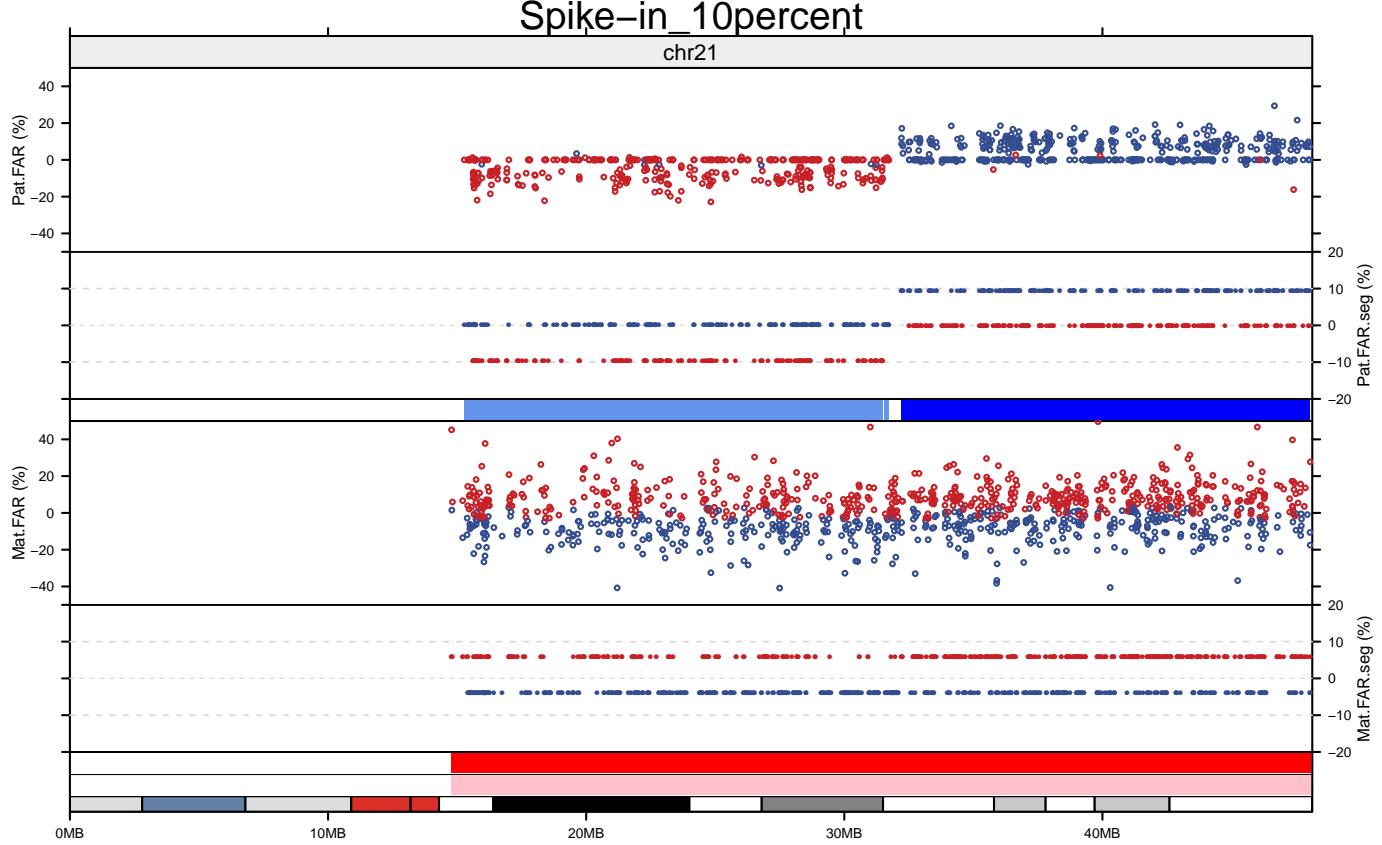

b

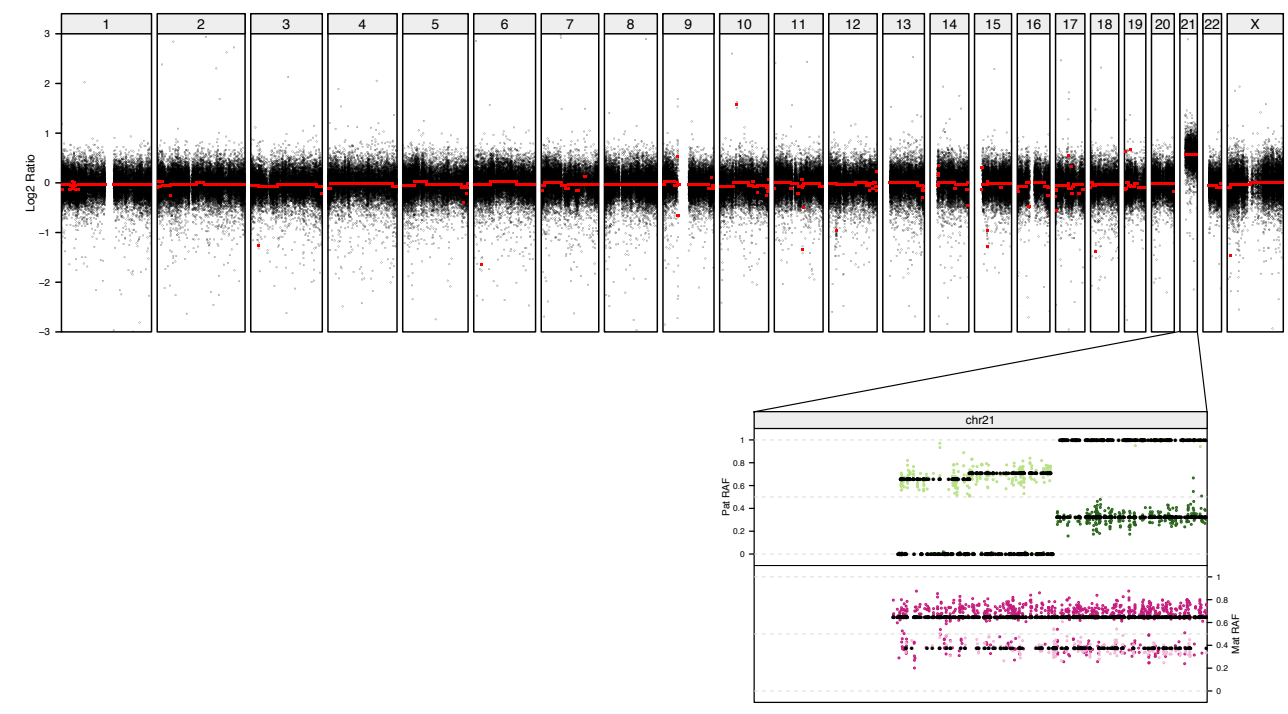




\section{Supplementary Figure S1}

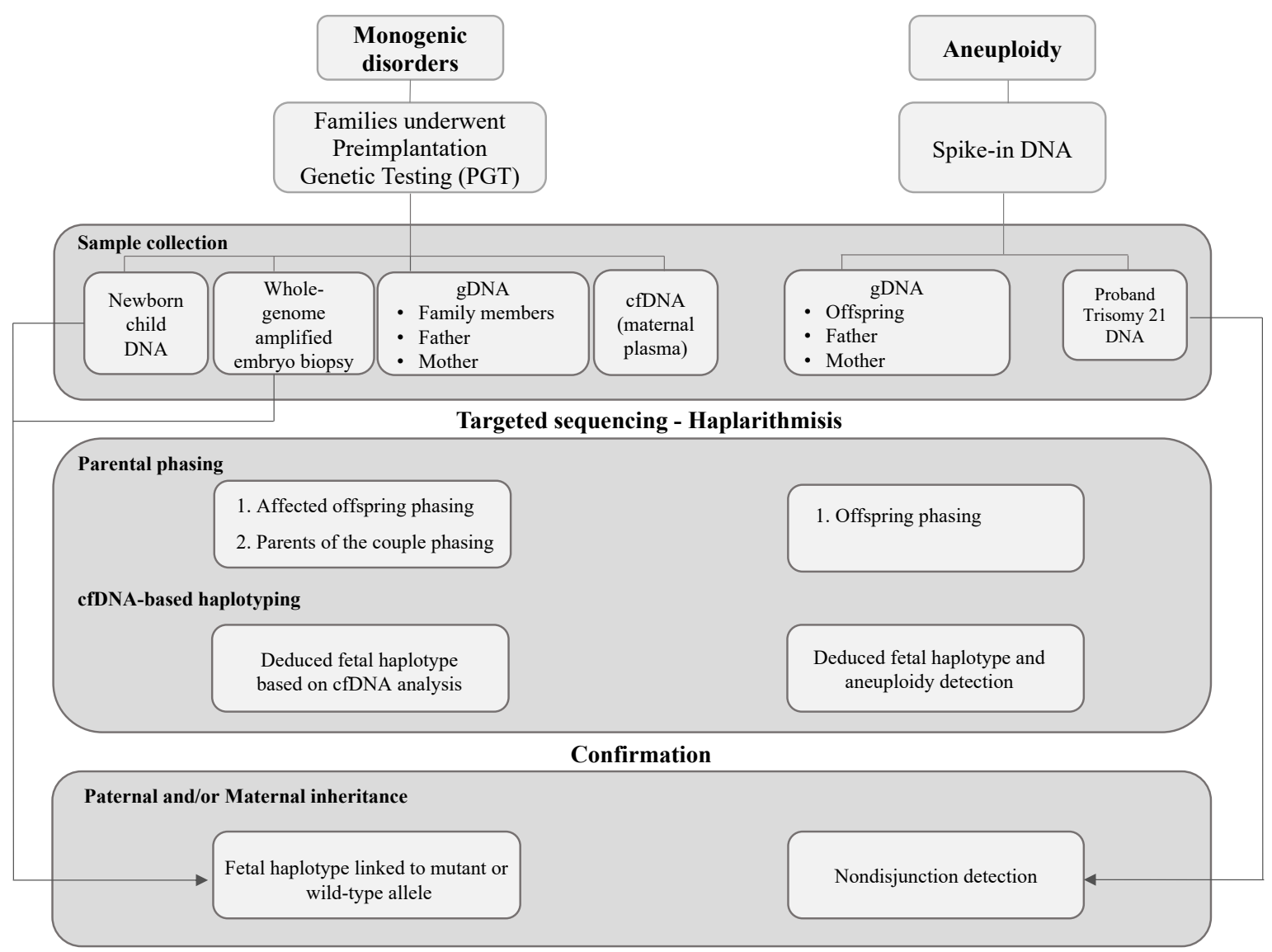




\section{Supplementary Figure S2}

Paternal Parents for Phasing

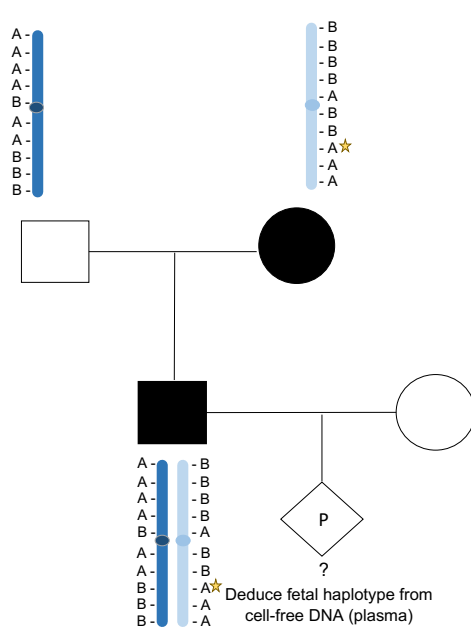

Maternal Parents for Phasing

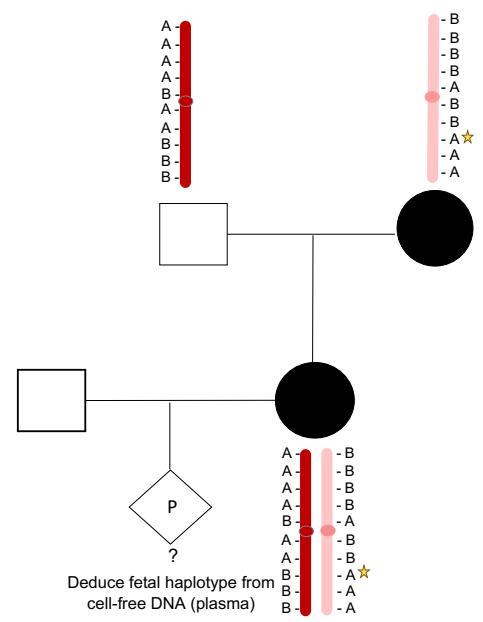




\section{Supplementary Figure S3}

a

Family3_085
Chromosome
$\square\|\|{ }_{25 \mathrm{MB}}^{6}$

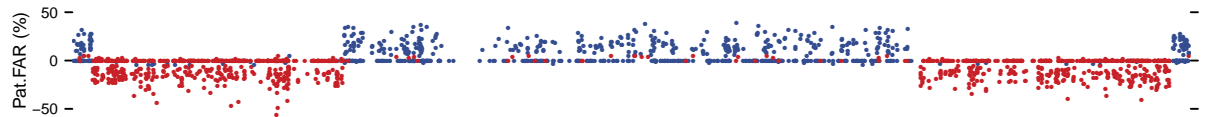

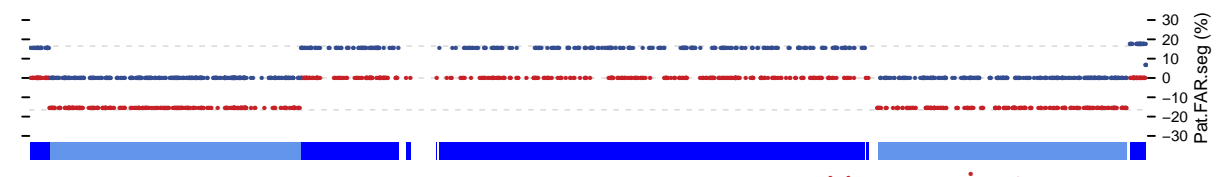

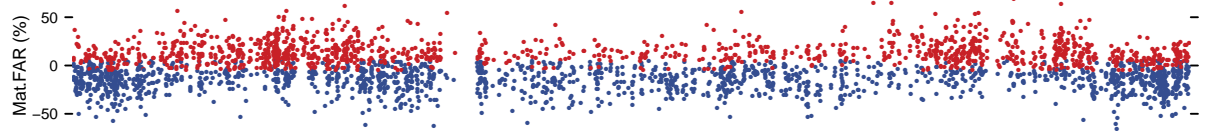

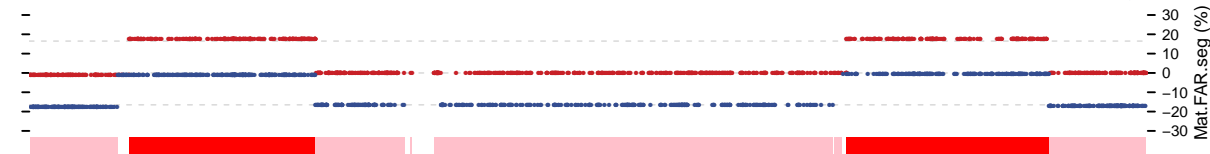

b

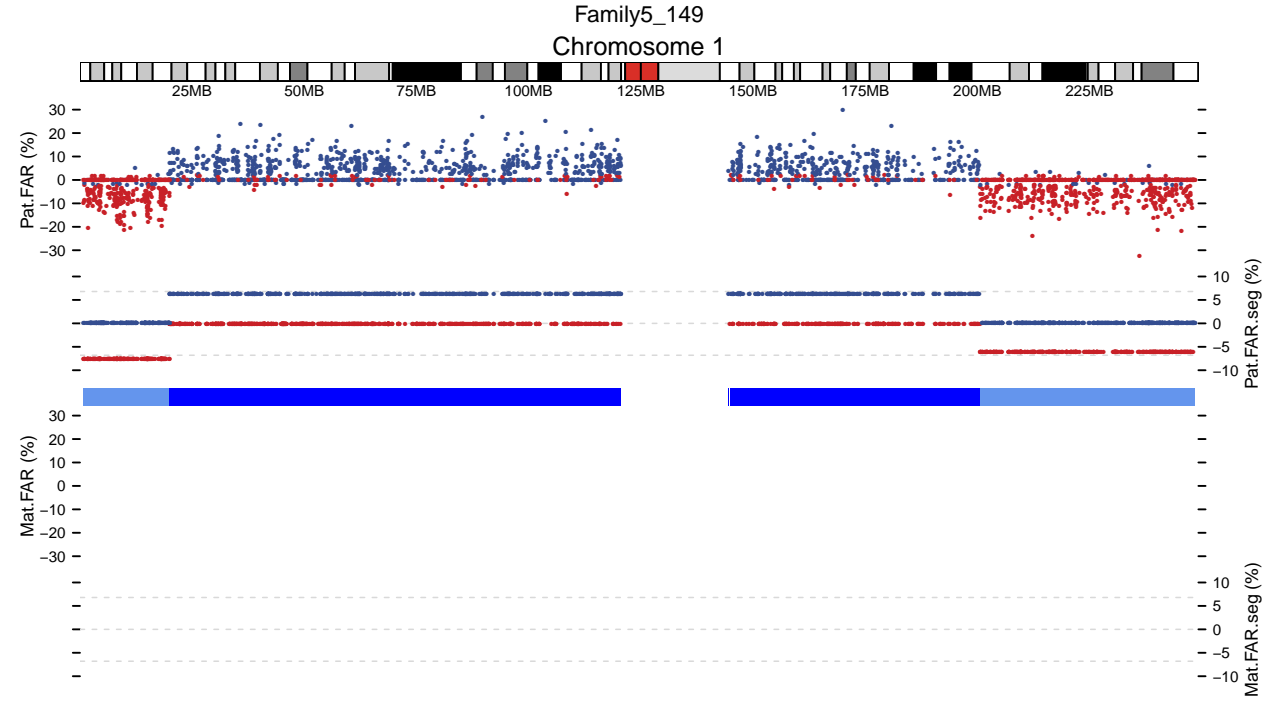

c

Family8 078

Chromosome 2
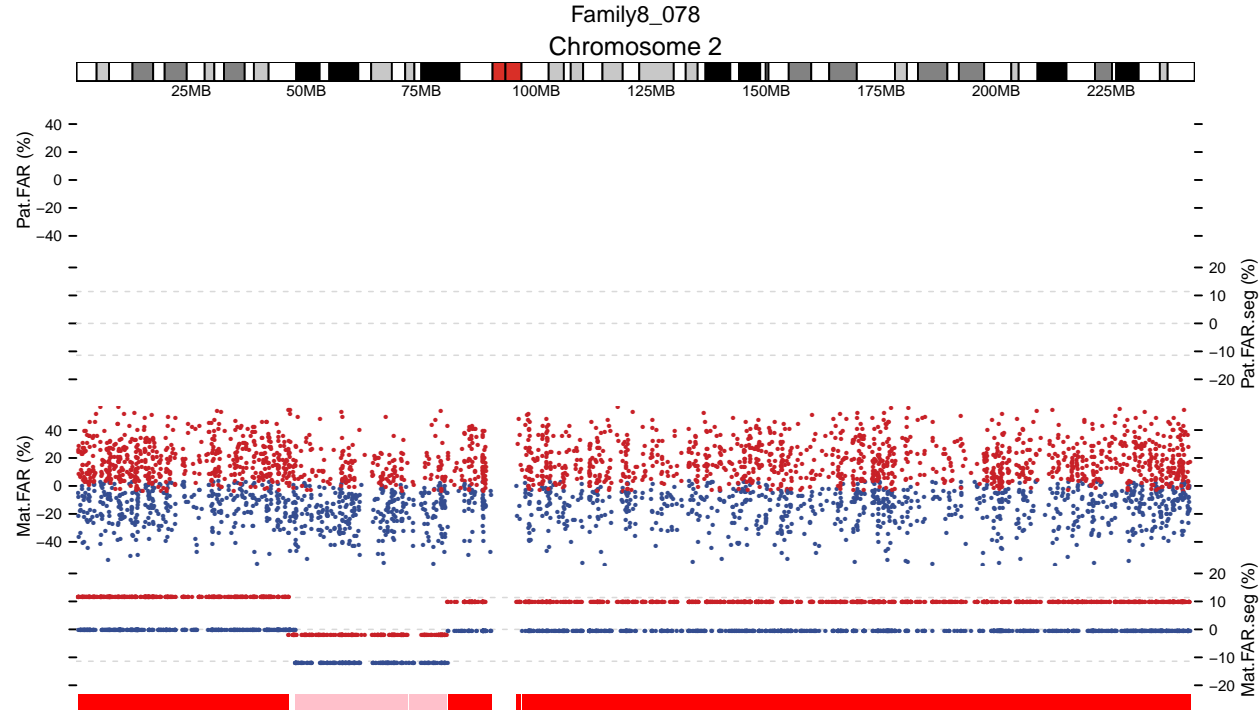
a

Family4_158

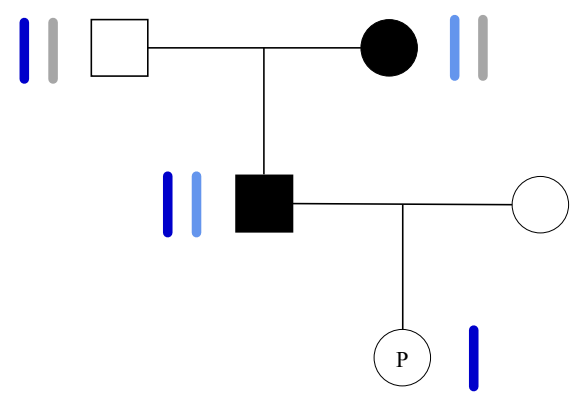

b

Family6_150

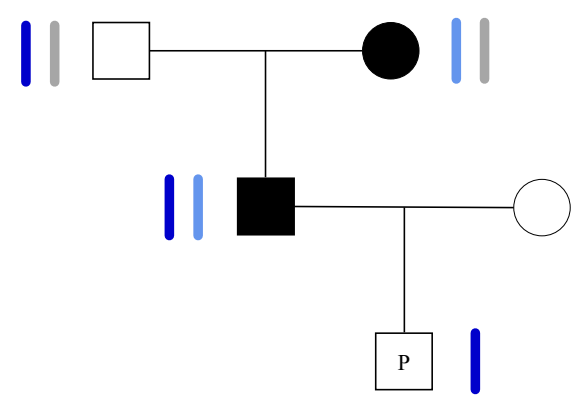

c
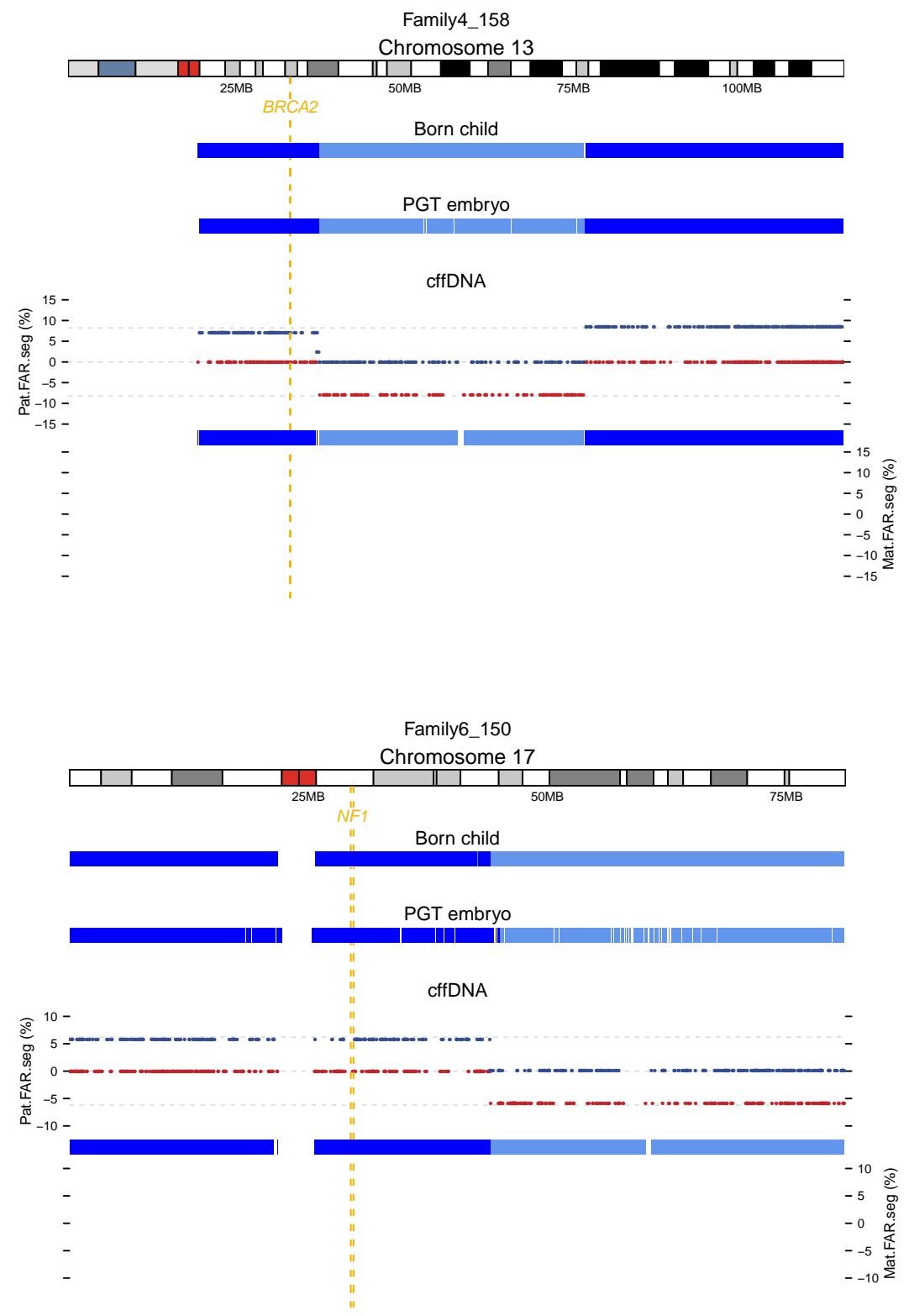

Family5_149

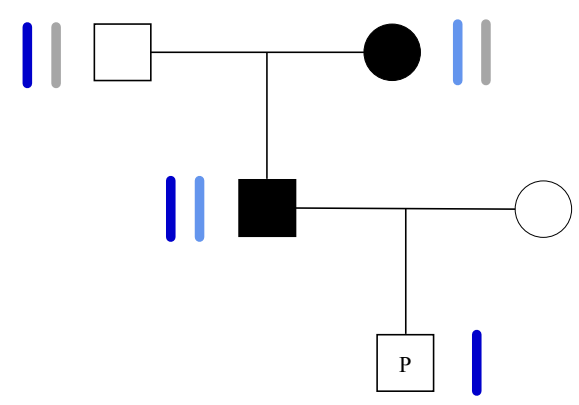

Family5_149

Chromosome 13




d

Family7_054

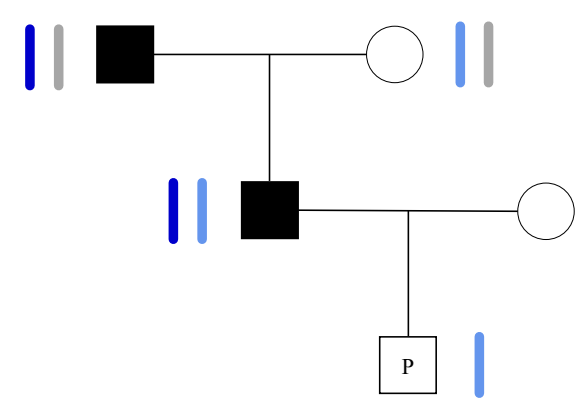

e

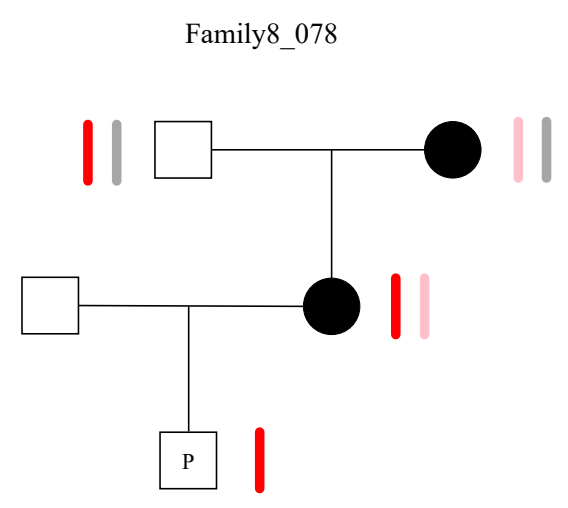

Family9_074
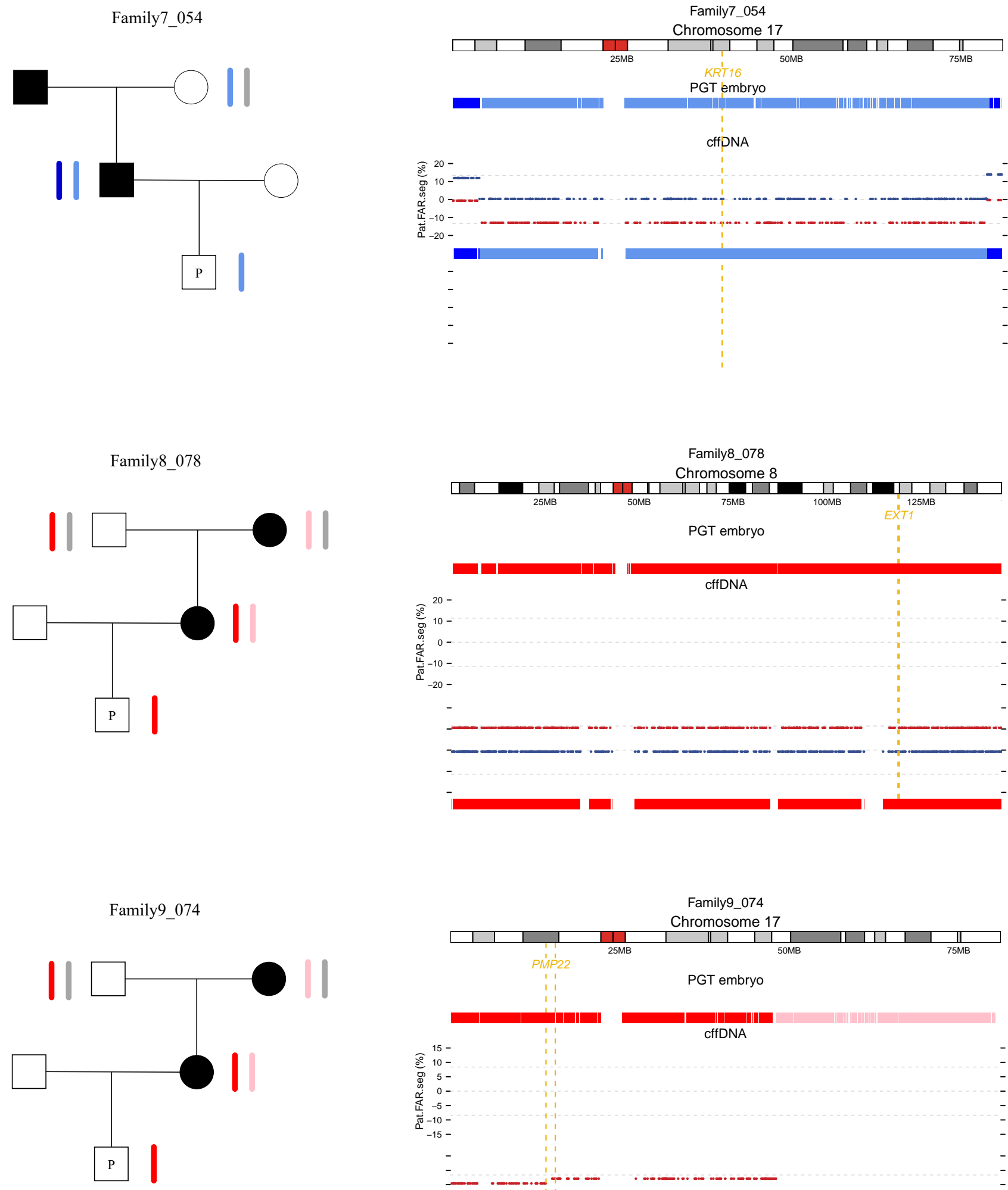

cffDNA

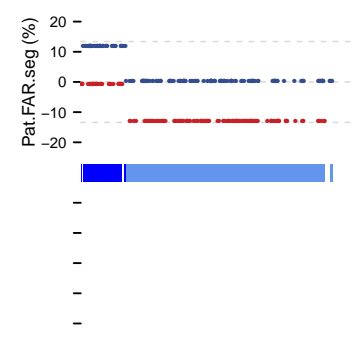

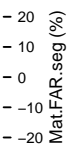

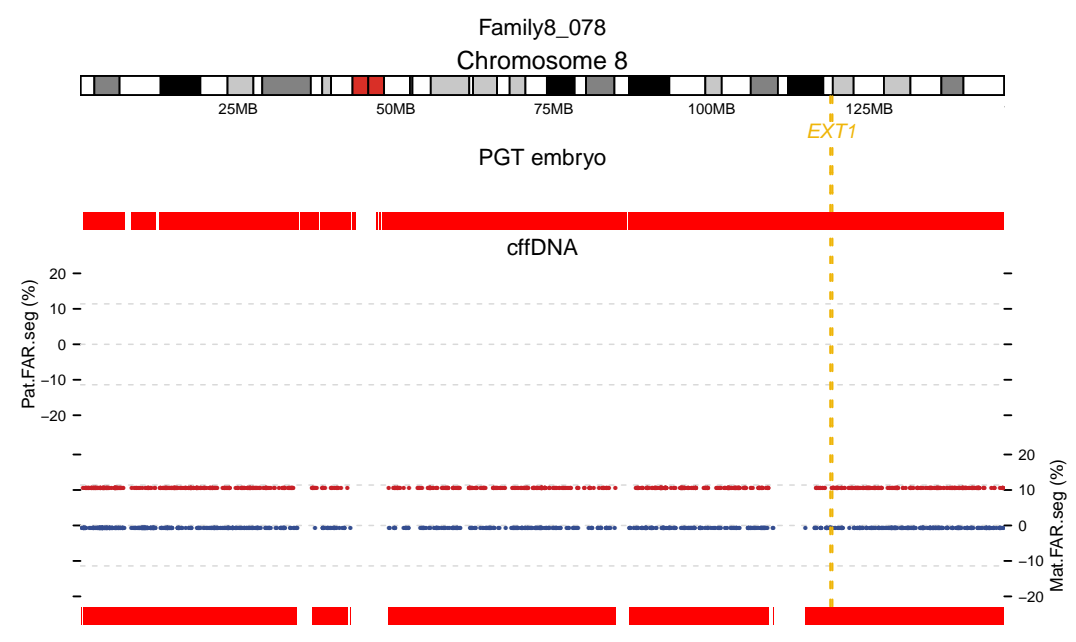

f 


\section{Supplementary Figure S5}

a

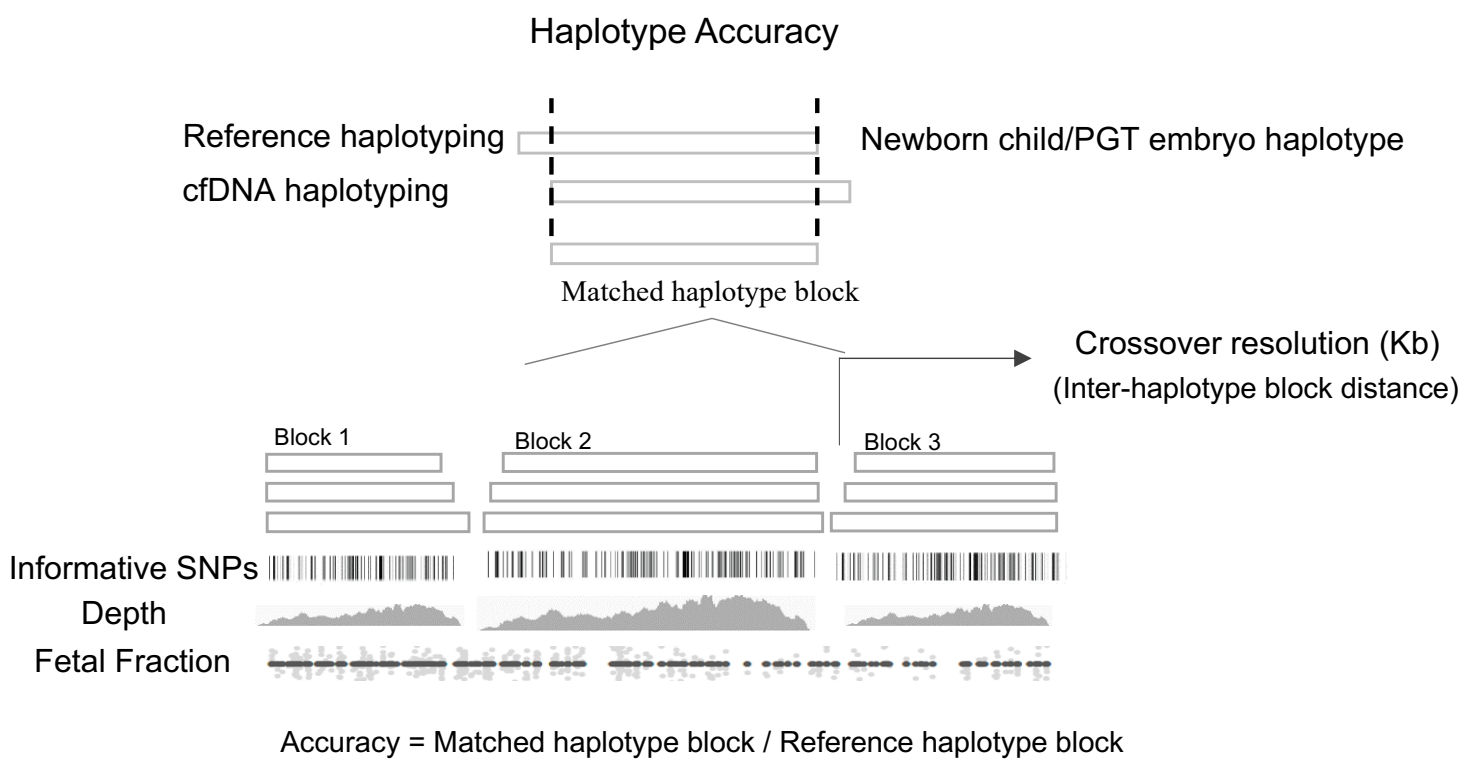

b

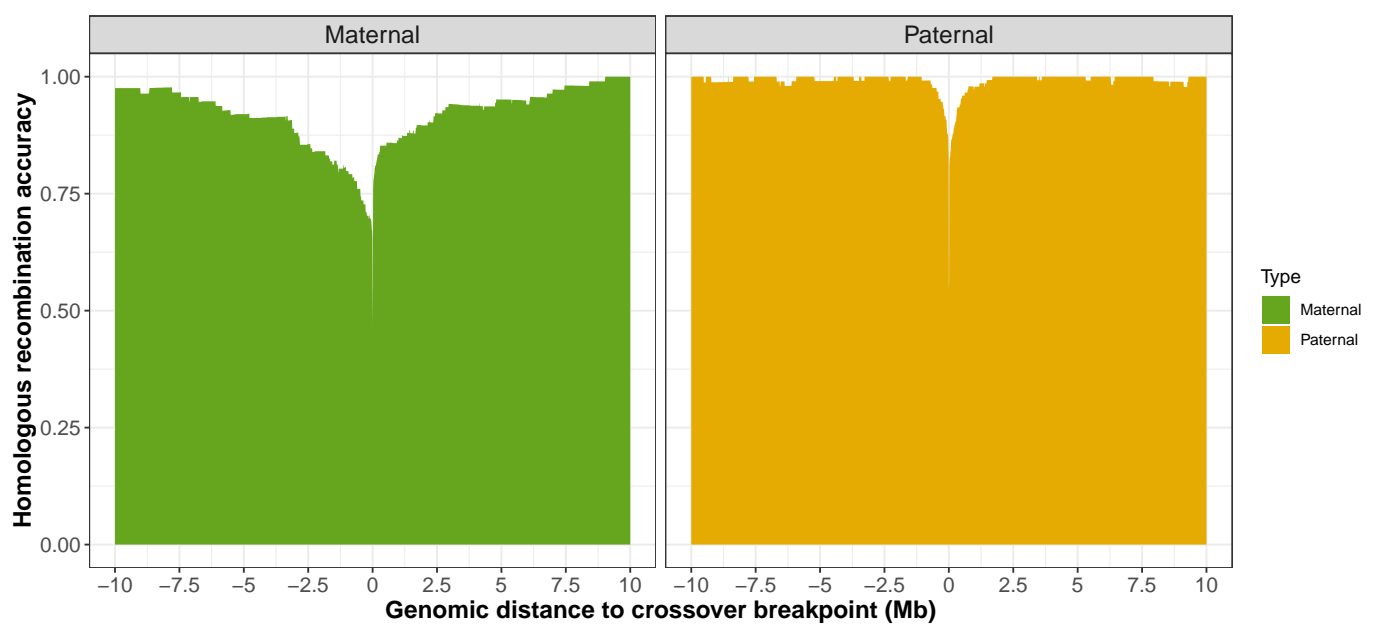

C

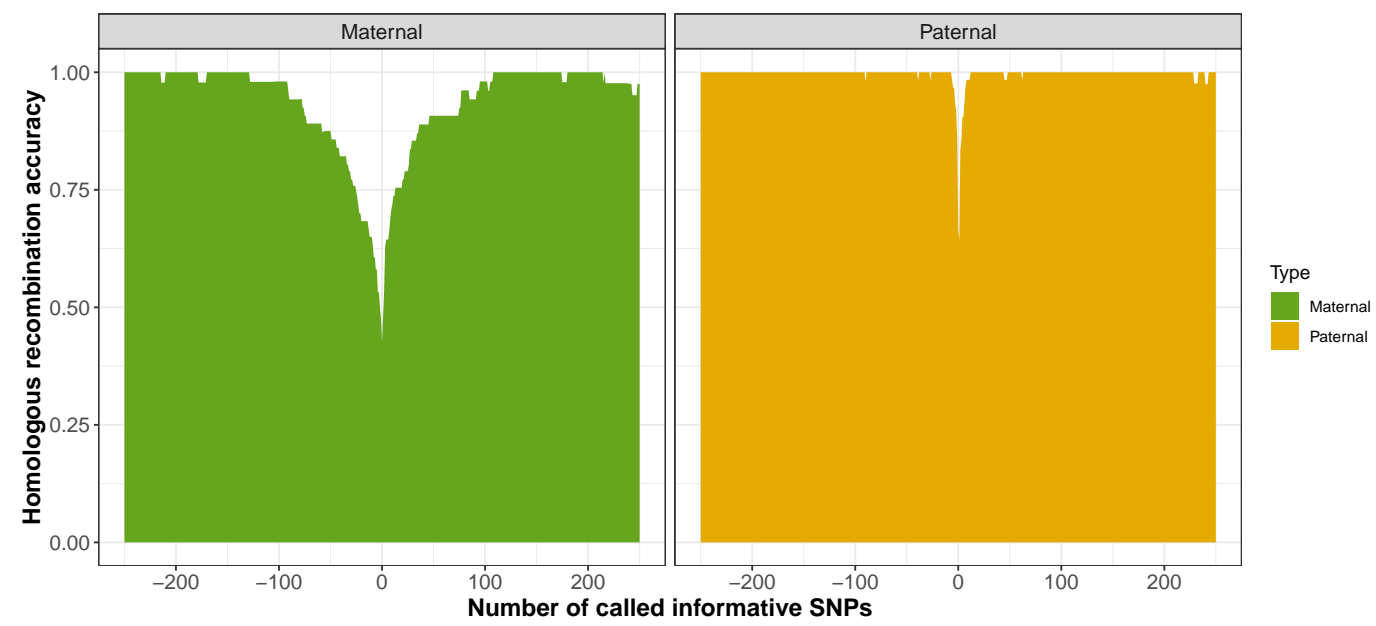




\section{Supplementary Figure S6}

a

Family4_158_Bornchild

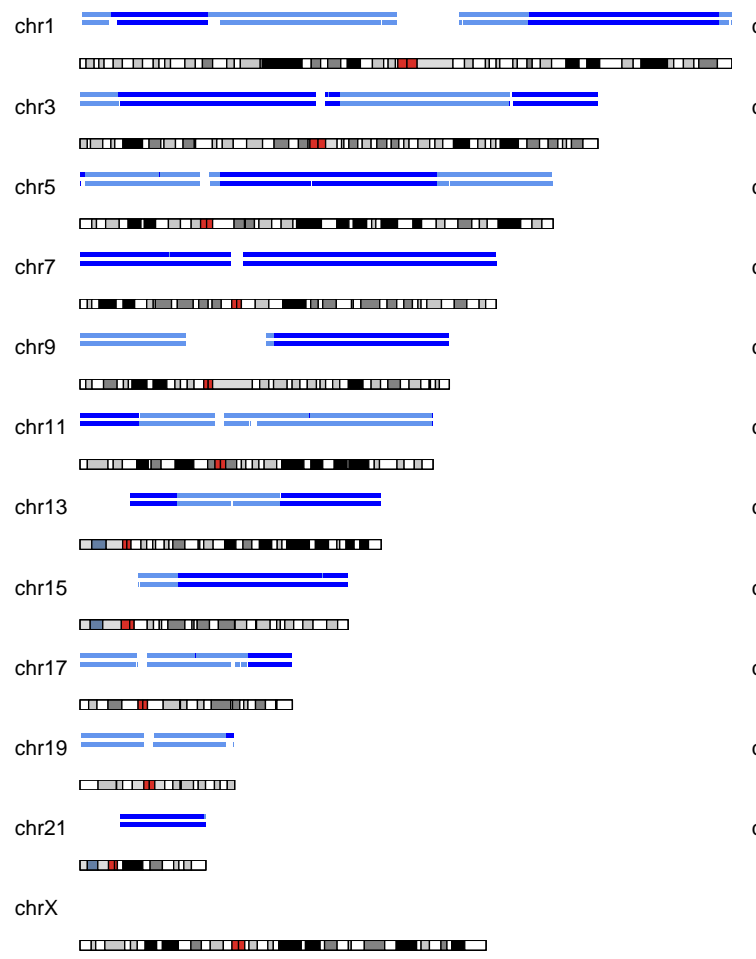

Family6_150_Bornchild 


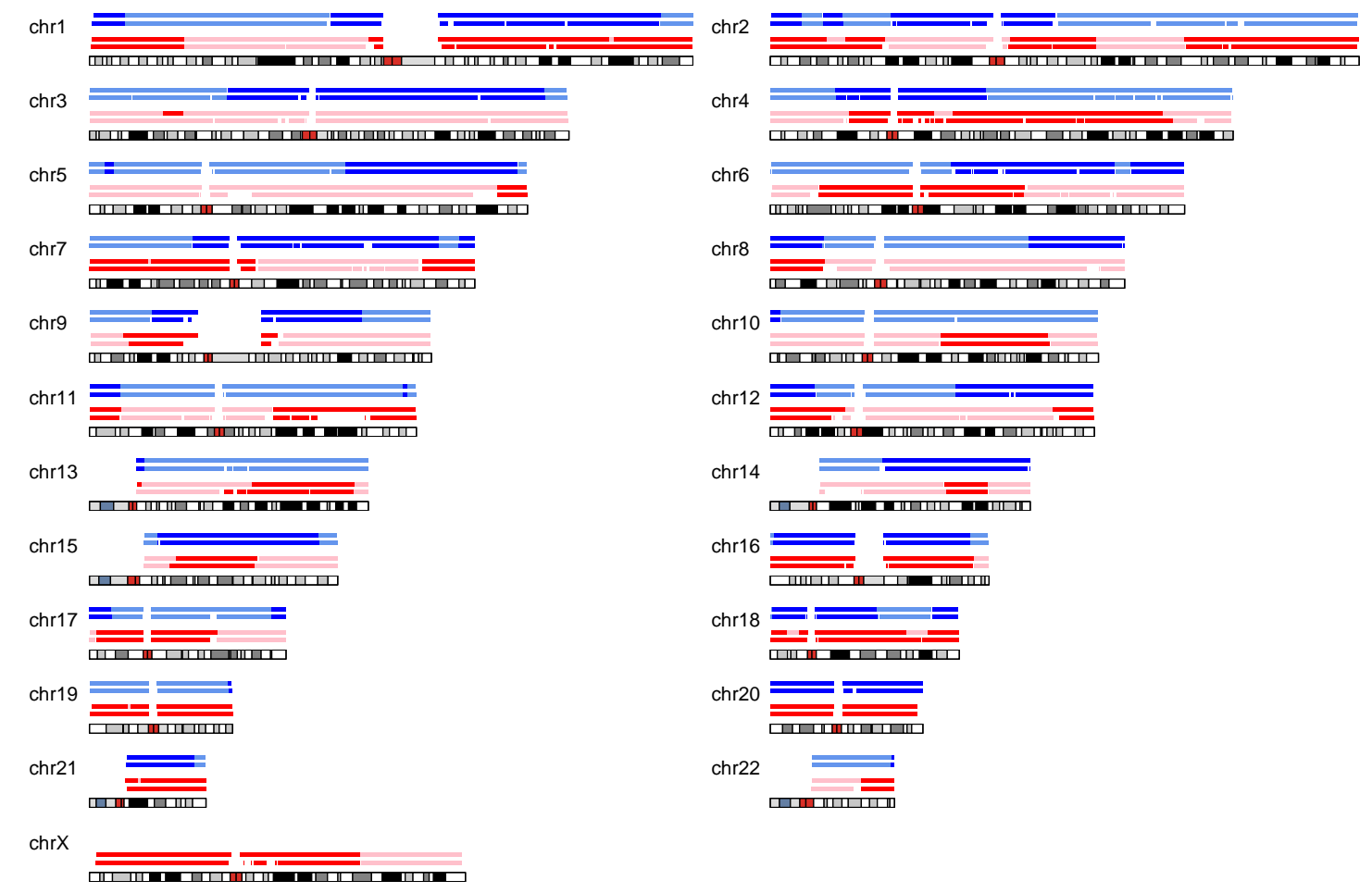
Family2_186_PGTembryo
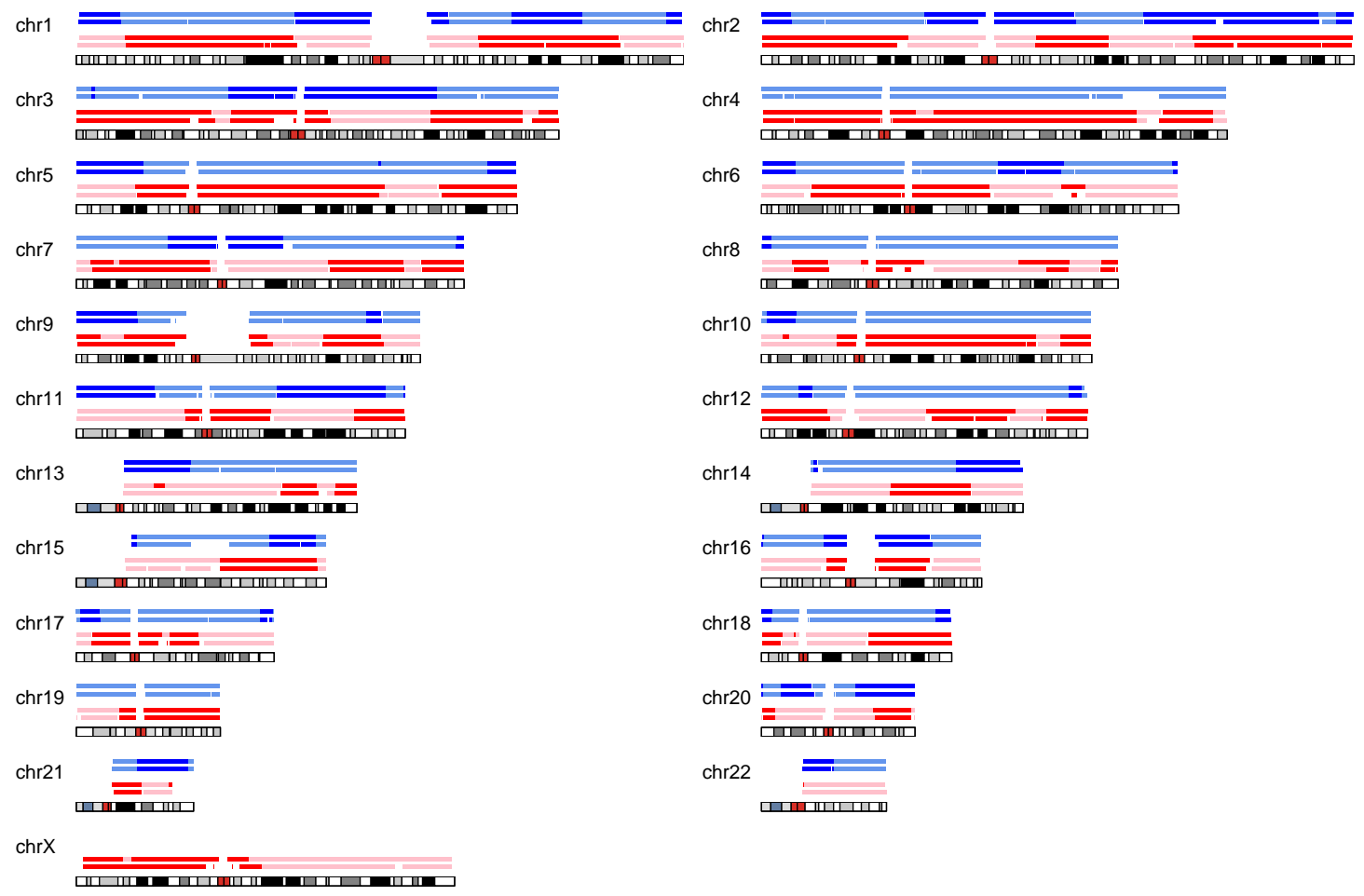
Family4_158_PGTembryo

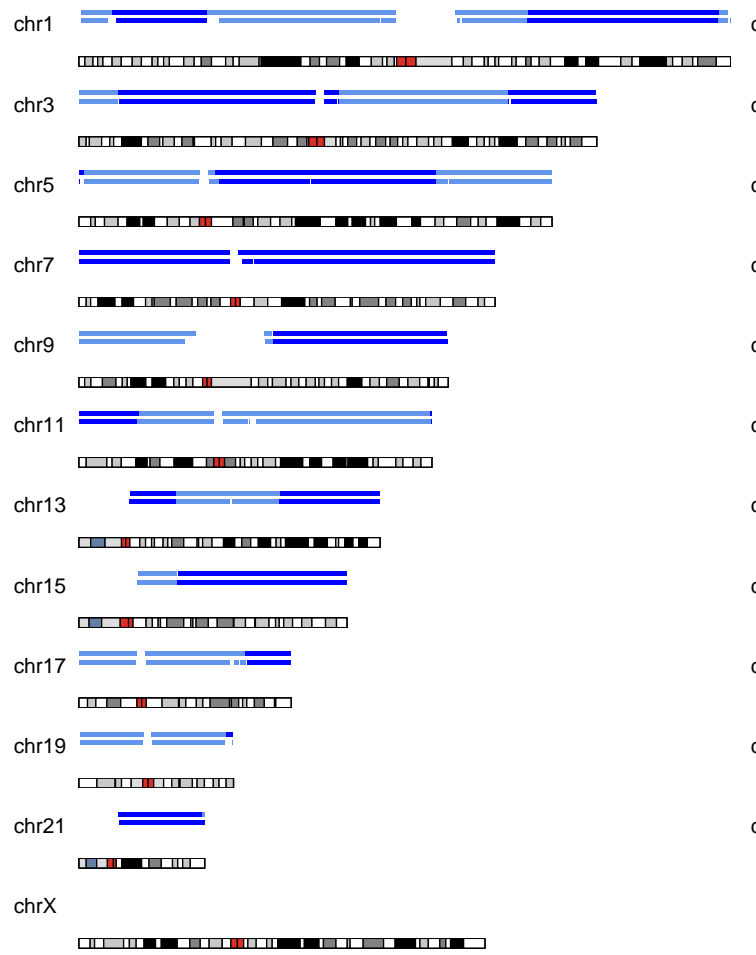

Family5_149_PGTembryo

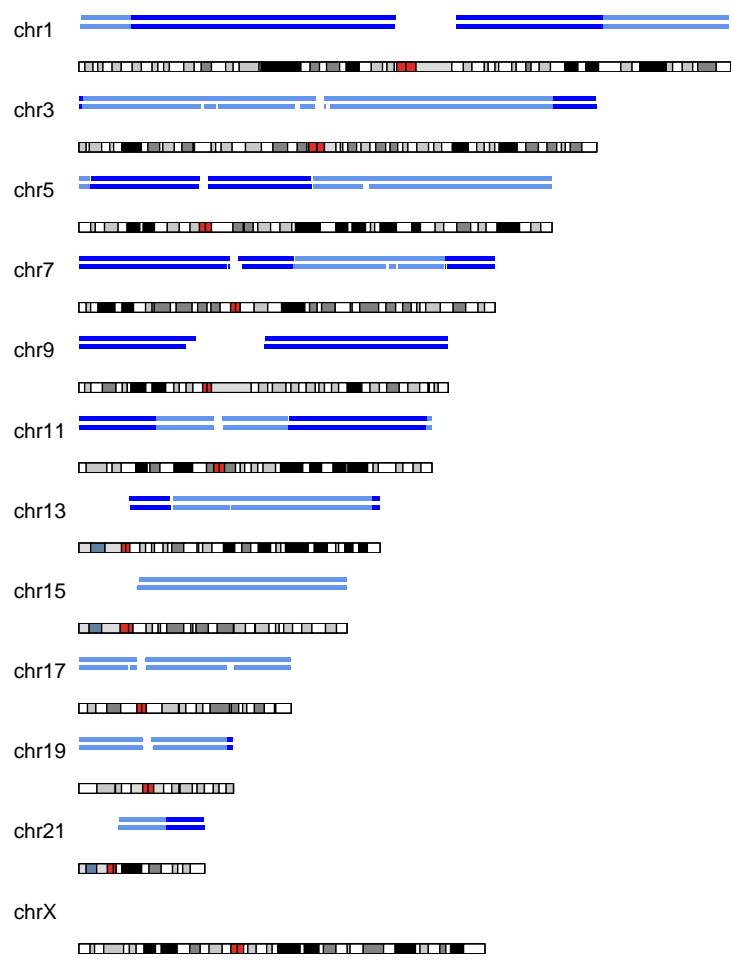


Family8_078_PGTembryo

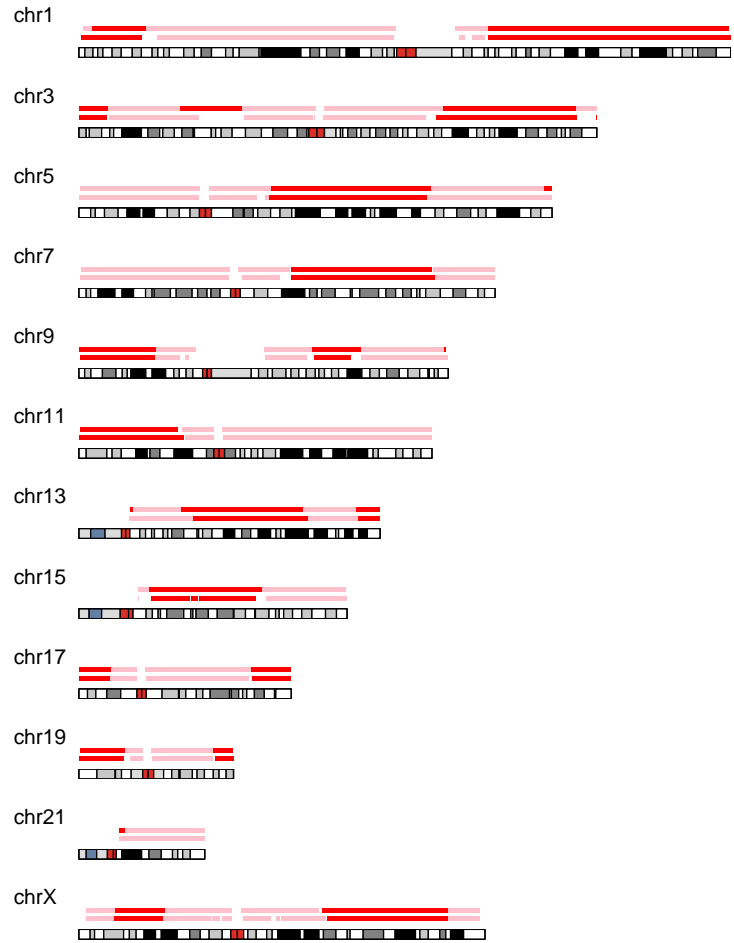

chr1

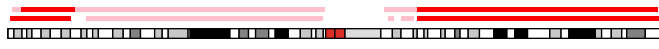

chr3

=

chr5

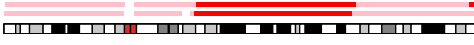

chr

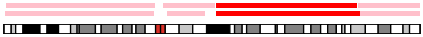

chr9

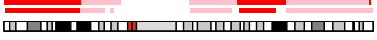

chr11

سلمس

chr13

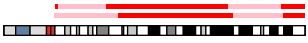

chr15

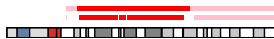

chr17

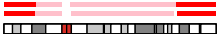

chr19

$\overline{\overline{1}}=\overline{\overline{10}}=$

chr21

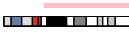

$\operatorname{chr}$

$\overline{\overline{10}}$

chr2

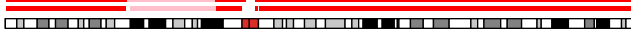

chr4

मान

chr6

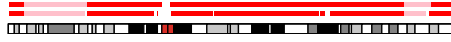

chr8

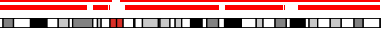

chr10

ला1

chr12

=

chr14

п

chr16

पालान 11

chr18

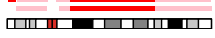

chr20

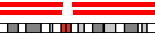

chr22

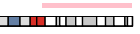

Family9_074_PGTembryo

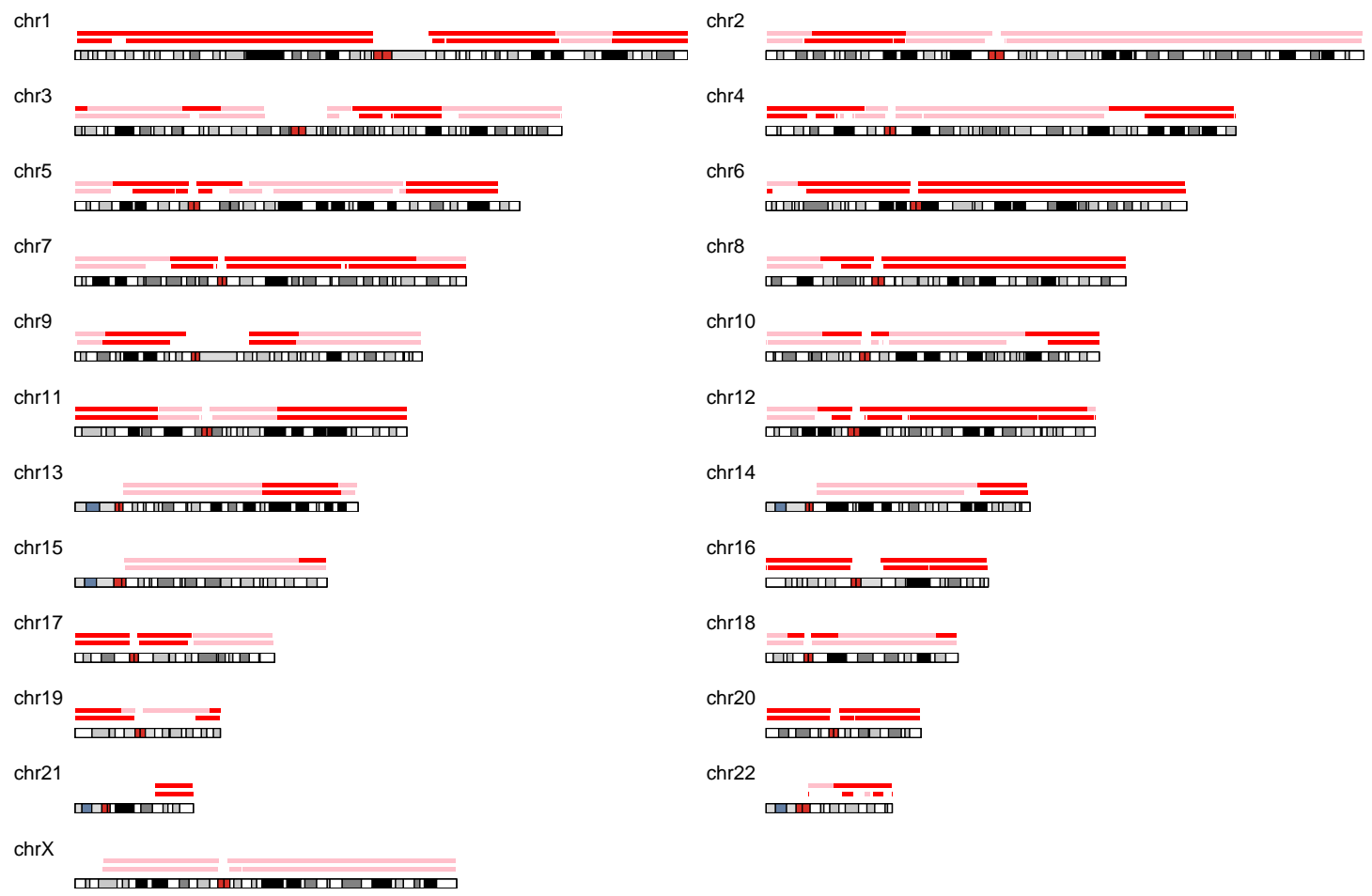




\section{Supplementary Figure S7}
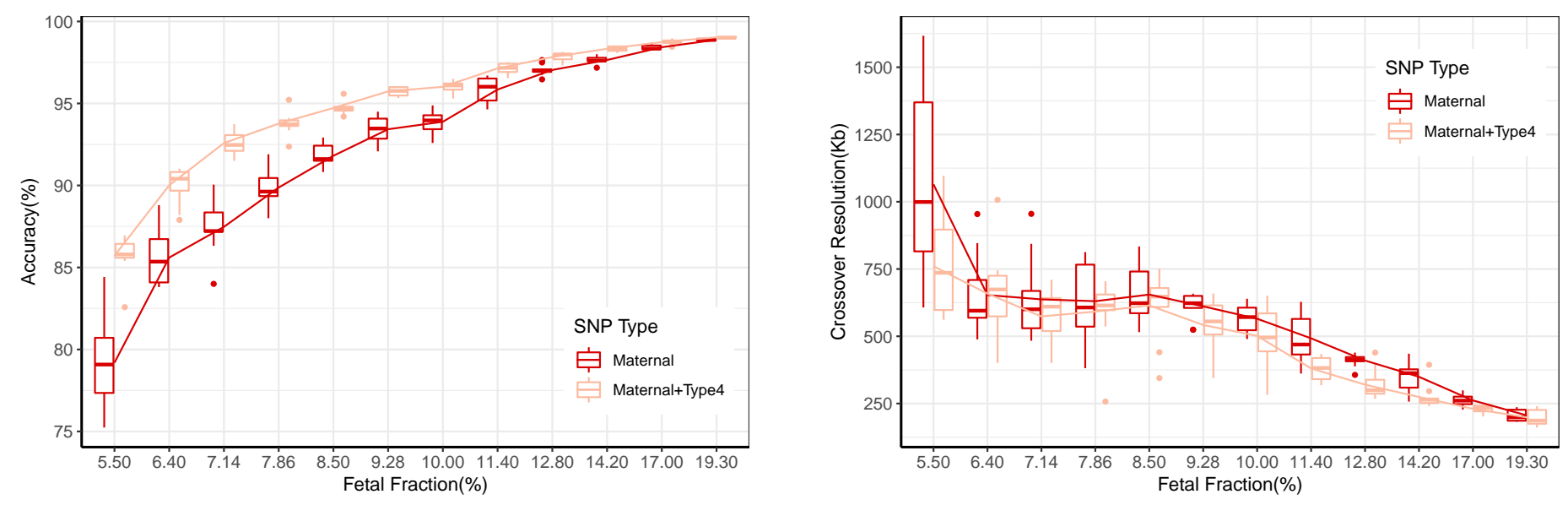


\section{Supplementary Figure S8}
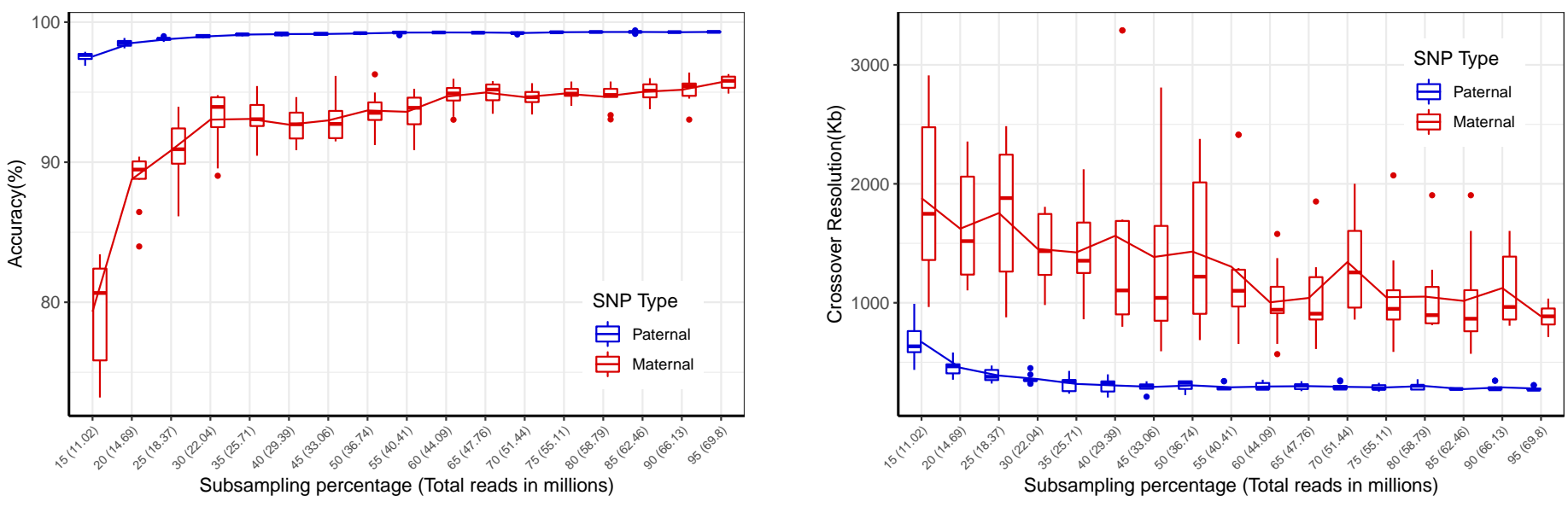


\section{Supplementary Figure S9}

a
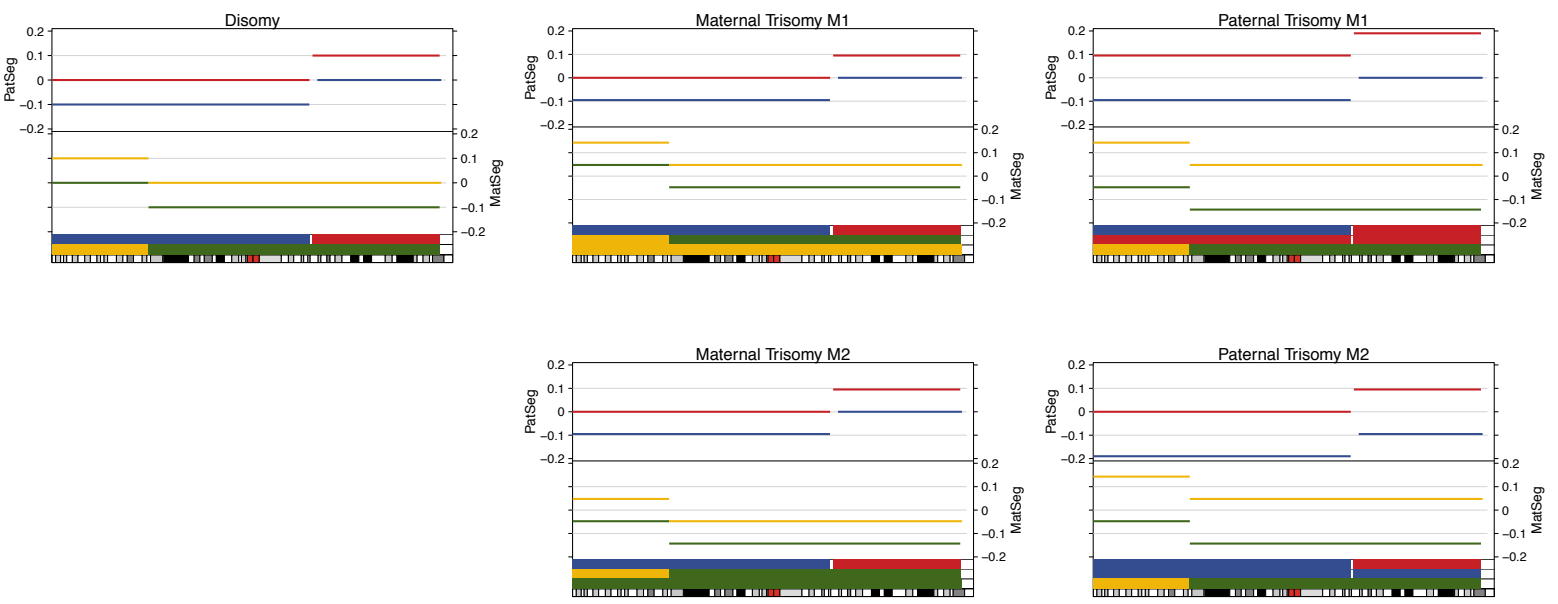

b

\begin{tabular}{|c|c|c|c|c|c|c|c|c|c|}
\hline \multirow[b]{2}{*}{ Paternal } & \multicolumn{3}{|c|}{ Normal Disomy } & \multicolumn{3}{|c|}{ Maternal Trisomy } & \multicolumn{3}{|c|}{ Paternal Trisomy } \\
\hline & Homolog & P1 & P2 & Homolog & P1 & P2 & Homolog & P1 & P2 \\
\hline & H1 & 0 & 0.1 & H1 & 0 & 0.0952 & $\mathrm{H} 1+\mathrm{H} 2$ & -0.0952 & 0.0952 \\
\hline & $\mathrm{H} 2$ & -0.1 & 0 & $\mathrm{H} 2$ & -0.0952 & 0 & $2 \mathrm{H} 1$ & 0 & 0.19 \\
\hline & & & & & & & $2 \mathrm{H} 2$ & 0.19 & 0 \\
\hline \multirow[t]{4}{*}{ Maternal } & Homolog & M1 & M2 & Homolog & M1 & M2 & Homolog & M1 & M2 \\
\hline & H1 & 0.1 & 0 & $\mathrm{H} 1+\mathrm{H} 2$ & 0.0476 & -0.0476 & H1 & 0.143 & -0.0476 \\
\hline & H2 & 0 & 0.1 & $2 \mathrm{H} 1$ & 0.143 & 0.0476 & $\mathrm{H} 2$ & 0.0476 & -0.143 \\
\hline & & & & $2 \mathrm{H} 2$ & -0.0476 & -0.143 & & & \\
\hline
\end{tabular}




\section{Supplementary Figure S10}

a

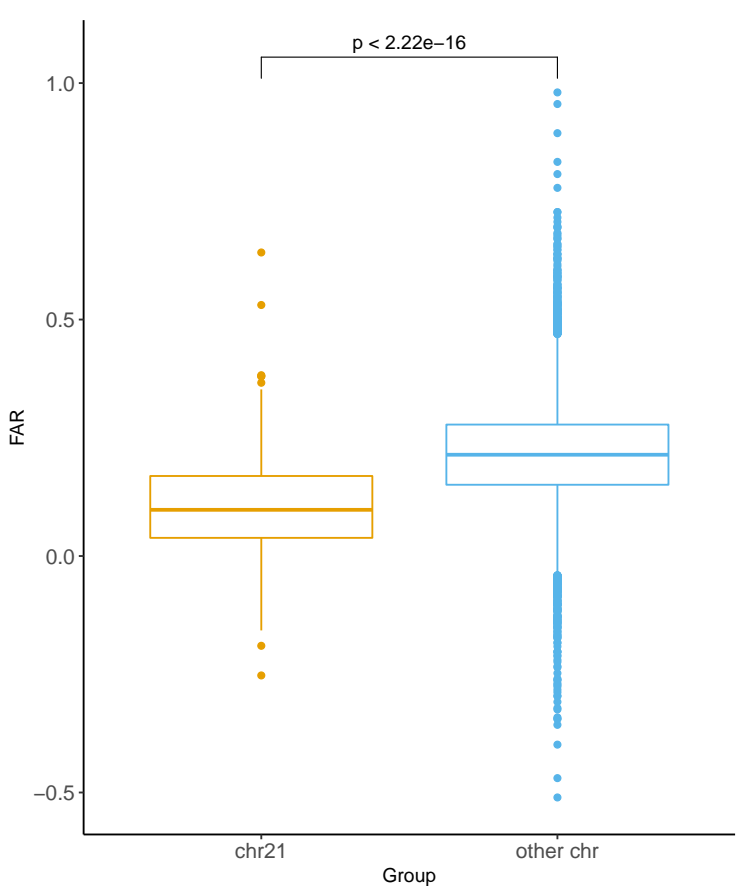

c

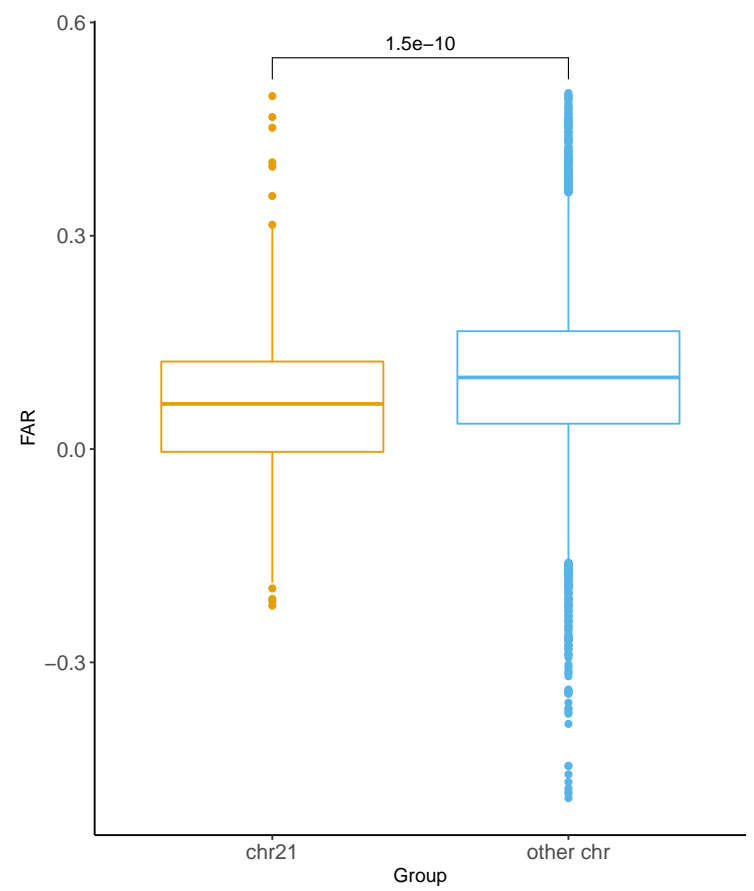

b

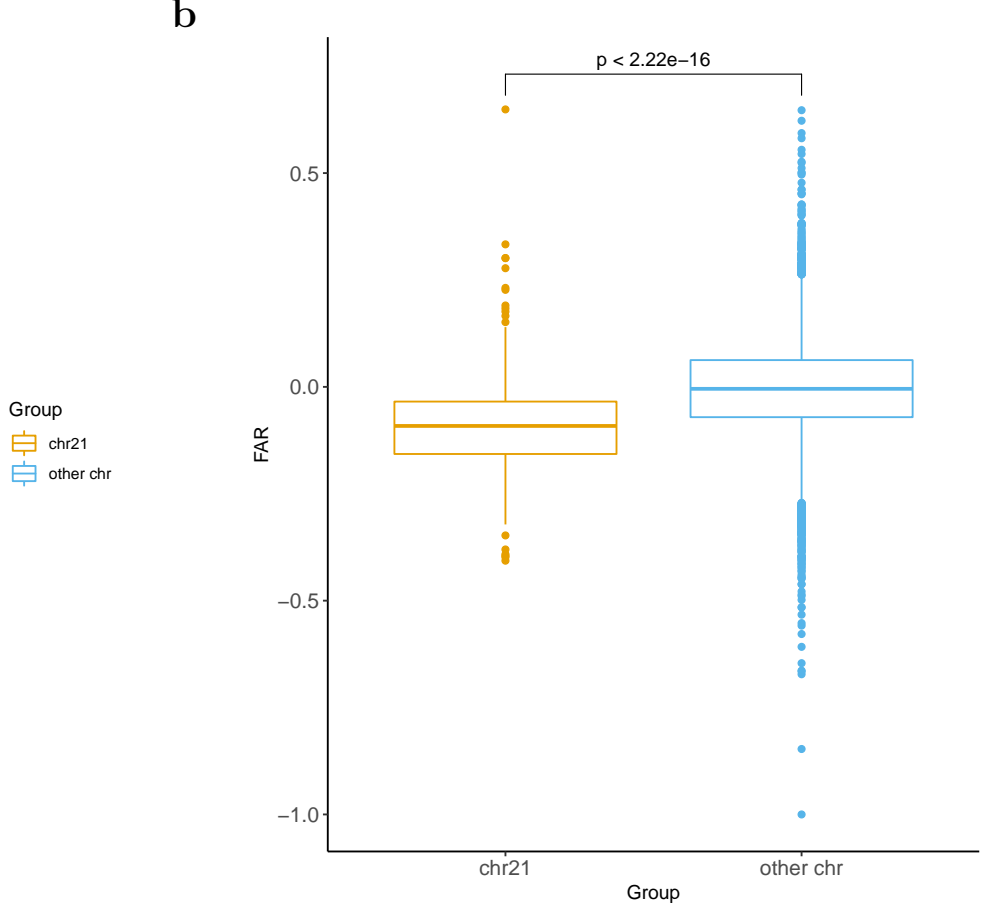

d






\section{Supplementary Figure S11}

a

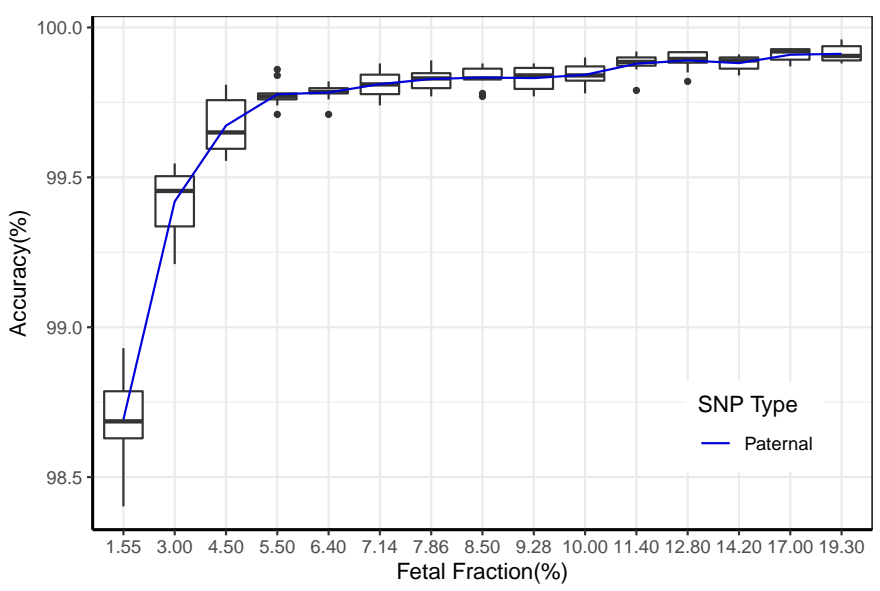

b

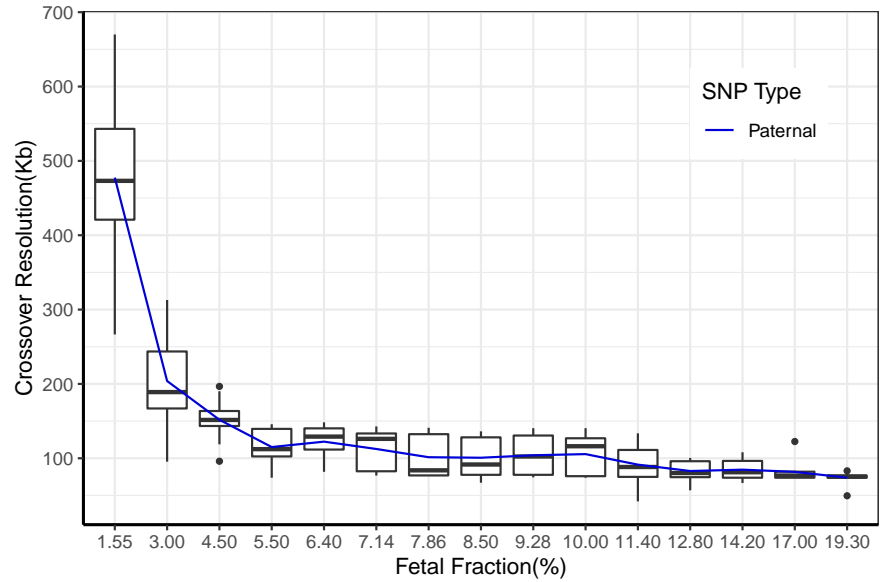




\section{Supplementary Figure S12}

a

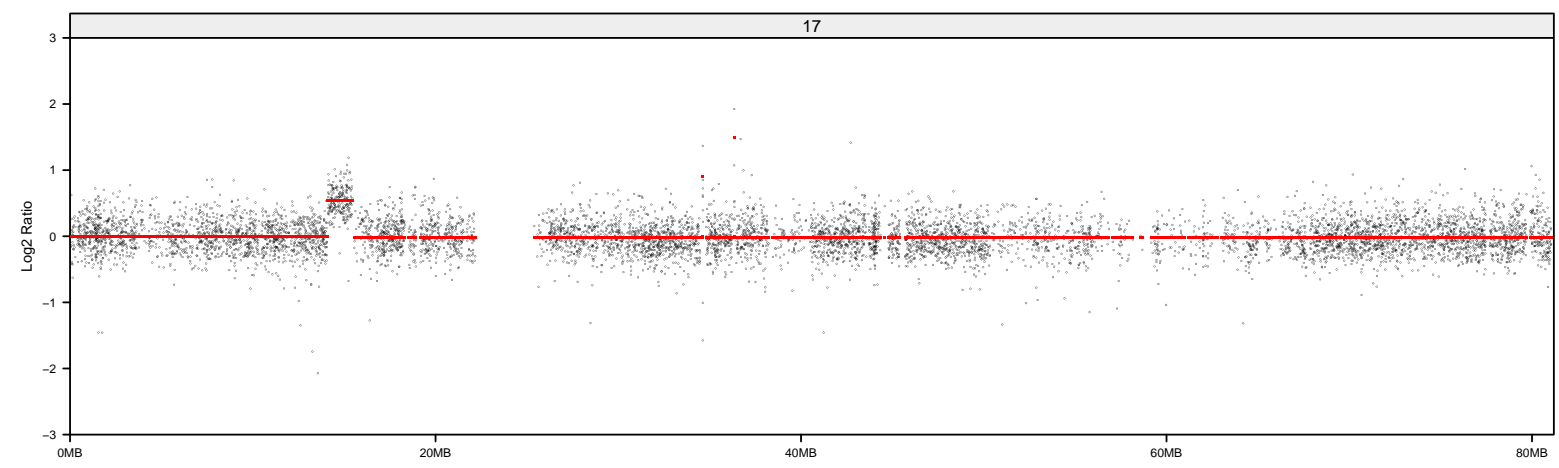

b

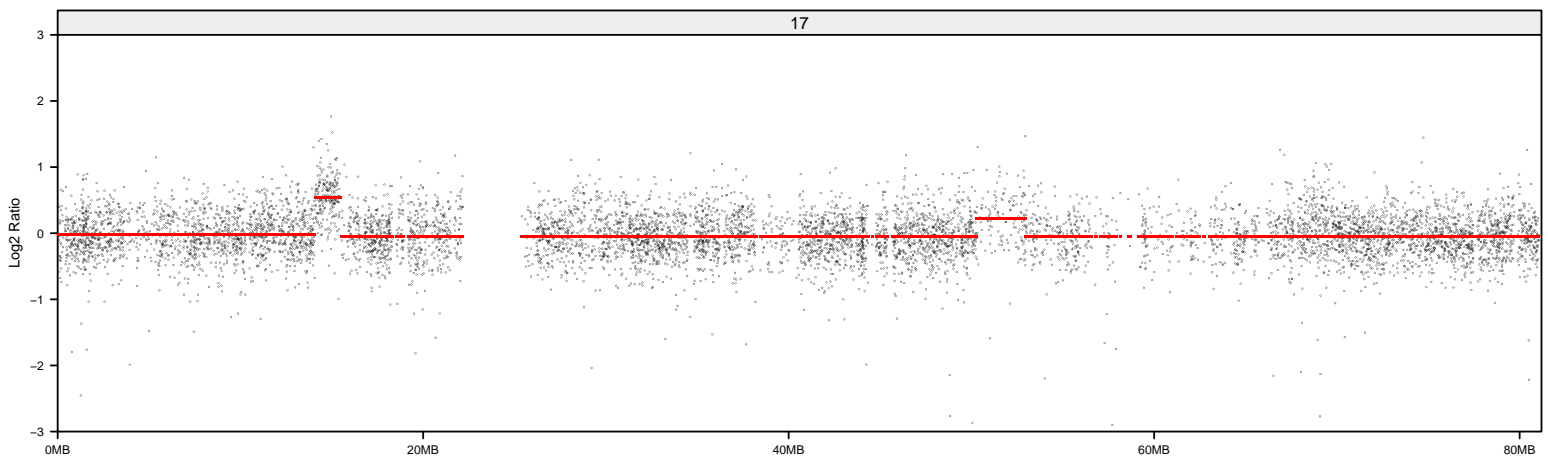




\section{Supplementary Figure S13}

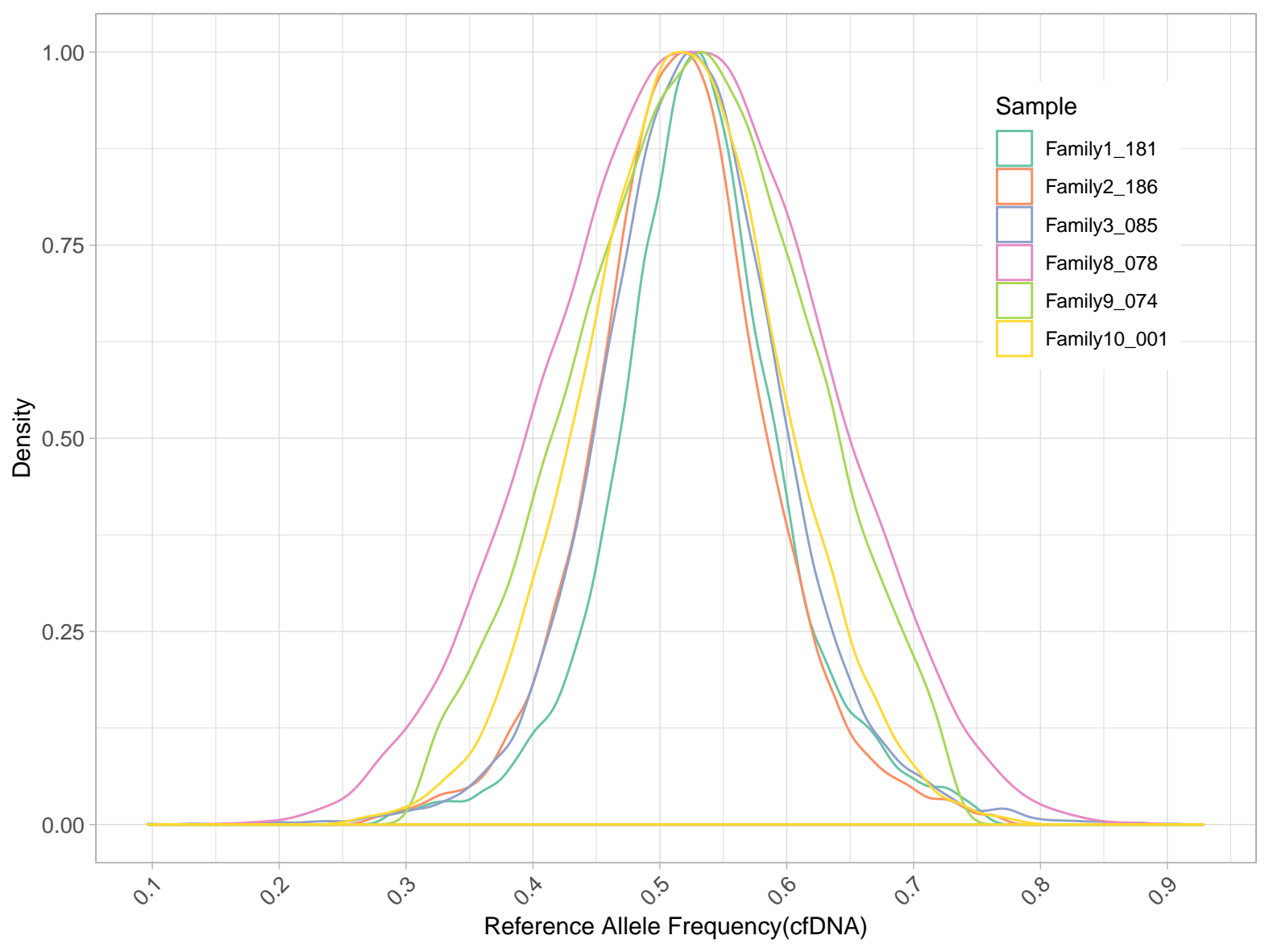


Table 1. Clinical information of all families that followed pre-implantation genetic testing (PGT)

\begin{tabular}{|c|c|c|c|c|c|c|c|c|c|c|c|c|}
\hline \multirow[t]{2}{*}{ Family } & \multirow[t]{2}{*}{ Disease } & \multirow[t]{2}{*}{ Gene } & \multirow{2}{*}{$\begin{array}{l}\text { Cytogenetic } \\
\text { Location }\end{array}$} & \multirow{2}{*}{$\begin{array}{l}\text { Genomic } \\
\text { coordinates }\end{array}$} & \multirow[t]{2}{*}{ MI } & \multicolumn{4}{|c|}{ Mutation } & \multirow[t]{2}{*}{ Phasing } & \multirow{2}{*}{$\begin{array}{l}\text { Fetal } \\
\text { sex }\end{array}$} & \multirow{2}{*}{$\begin{array}{l}\text { Gestation } \\
\text { age } \\
\text { (weeks) }\end{array}$} \\
\hline & & & & & & Mother & Father & Other family member & Fetus & & & \\
\hline 2_186 & $\begin{array}{l}\text { Gaucher disease, type } 1 \\
(\# 230800)\end{array}$ & $\begin{array}{l}G B A \\
\left(\mathrm{NM} \_000157.3\right)\end{array}$ & $1 \mathrm{q} 22$ & $\begin{array}{l}\operatorname{chr} 1: 155,204,238- \\
155,214,652\end{array}$ & $\mathrm{AR}$ & $\begin{array}{l}\text { Heterozygous c. } 1448 \mathrm{~T}>\mathrm{C} \\
\text { (p.Leu483Pro) }\end{array}$ & $\begin{array}{l}\text { Heterozygous c.1448T>C } \\
\text { (p.Leu483Pro) }\end{array}$ & $\begin{array}{l}\text { Sbling homozygous } \\
\text { c. } 1448 T>C \text { (p.Leu } 483 \text { Pro) }\end{array}$ & $\begin{array}{l}\text { Non } \\
\text { carrier }\end{array}$ & $\begin{array}{l}\text { Affected } \\
\text { offspring }\end{array}$ & $\mathrm{F}$ & 12 \\
\hline 3_085 & $\begin{array}{l}\text { Chondrodysplasia } \\
\text { punctata, X-linked } \\
\text { dominant (\#302960) }\end{array}$ & $\begin{array}{l}E B P \\
\text { (NM_006579.2) }\end{array}$ & Xp11.23 & $\begin{array}{l}\text { chrX:48,379,546- } \\
48,387,104\end{array}$ & XLD & $\begin{array}{l}\text { Heterozygous c.116C >G } \\
\text { (p.Thr39Arg) }\end{array}$ & Non carrier & $\begin{array}{l}\text { Sibling heterozygous } \\
\text { c. } 116 \mathrm{C}>\mathrm{G} \text { (p.Thr39Arg) }\end{array}$ & $\begin{array}{l}\text { Non } \\
\text { carrier }\end{array}$ & $\begin{array}{l}\text { Affected } \\
\text { offspring }\end{array}$ & M & 12 \\
\hline 4_158 & $\begin{array}{l}\text { Breast-ovarian cancer, } \\
\text { familial, } 2 \text { (\#612555) }\end{array}$ & $\begin{array}{l}\text { BRCA2 } \\
\text { (NM_000059.3) }\end{array}$ & $13 q 13.1$ & $\begin{array}{l}\text { chr13:32,889,616- } \\
32,973,808\end{array}$ & $\mathrm{AD}$ & Non carrier & $\begin{array}{l}\text { Heterozygous c.5645C>A } \\
\text { (p.Ser1882*) }\end{array}$ & $\begin{array}{l}\text { Paternal grandmother } \\
\text { heterozygous c.5645C }>\text { A } \\
\text { (p.Ser1882*) }\end{array}$ & $\begin{array}{l}\text { Non } \\
\text { carrier }\end{array}$ & $\begin{array}{l}\text { Paternal } \\
\text { parents }\end{array}$ & $\mathrm{F}$ & 12 \\
\hline $6 \_150$ & $\begin{array}{l}\text { Neurofibromatosis, } \\
\text { type } 1 \text { (\#162200) }\end{array}$ & $\begin{array}{l}\text { NF1 } \\
\text { (NM_000267.3) }\end{array}$ & $17 \mathrm{q} 11.2$ & $\begin{array}{l}\text { chr17:29,421,944- } \\
29,704,694\end{array}$ & $\mathrm{AD}$ & Non carrier & $\begin{array}{l}\text { Heterozygous } \\
\text { c.7096_7101delAACTTT }\end{array}$ & $\begin{array}{l}\text { Paternal grandmother } \\
\text { heterozygous } \\
\text { c.7096_7101delAACTTT }\end{array}$ & $\begin{array}{l}\text { Non } \\
\text { carrier }\end{array}$ & $\begin{array}{l}\text { Paternal } \\
\text { parents }\end{array}$ & M & 13 \\
\hline 7_054 & $\begin{array}{l}\text { Pachyonychia } \\
\text { Congenita (\#167200) }\end{array}$ & $\begin{array}{l}\text { KRT16 } \\
\text { (NM_005557.3) }\end{array}$ & $17 \mathrm{q} 21.2$ & $\begin{array}{l}\operatorname{chr} 17: 39,766,030- \\
39,772,151\end{array}$ & $\mathrm{AD}$ & Non carrier & $\begin{array}{l}\text { Heterozygous c.374A }>\mathrm{G} \\
\text { (p.Asn125Ser) }\end{array}$ & $\begin{array}{l}\text { Paternal grandfather } \\
\text { heterozygous c.374A }>\mathrm{G} \\
\text { (p.Asn125Ser) }\end{array}$ & $\begin{array}{l}\text { Non } \\
\text { carrier }\end{array}$ & $\begin{array}{l}\text { Paternal } \\
\text { parents }\end{array}$ & M & 12 \\
\hline 8_078 & $\begin{array}{l}\text { Exostoses multiple } \\
\text { type } 1(\# 133700)\end{array}$ & $\begin{array}{l}\text { EXT1 } \\
\text { (NM_000127) }\end{array}$ & $8 \mathrm{q} 24.11$ & $\begin{array}{l}\text { chr8:118,806,729- } \\
119,124,092\end{array}$ & $\mathrm{AD}$ & $\begin{array}{l}\text { Heterozygous } \\
\text { c. } 2133 \_2151 \text { del }\end{array}$ & Non carrier & $\begin{array}{l}\text { Maternal grandmother } \\
\text { heterozygous c.2133_2151del }\end{array}$ & $\begin{array}{l}\text { Non } \\
\text { carrier }\end{array}$ & $\begin{array}{l}\text { Maternal } \\
\text { parents }\end{array}$ & M & 12 \\
\hline 9_074 & $\begin{array}{l}\text { Charcot-Marie-Tooth } \\
\text { disease type } 1 \\
(\# 118220)\end{array}$ & $\begin{array}{l}\text { CMT1A } \\
\text { syndrome region } \\
\text { including } P M P 22\end{array}$ & 17p12-p11 & $\begin{array}{l}\text { chr17:14,097,915- } \\
15,470,903\end{array}$ & $\mathrm{AD}$ & $\begin{array}{l}\text { Carrier of the common } \\
\sim 1.5 \mathrm{Mb} \text { duplication }\end{array}$ & Non carrier & $\begin{array}{l}\text { Maternal grandmother carrier } \\
\text { of the common } \sim 1.5 \mathrm{Mb} \\
\text { duplication }\end{array}$ & $\begin{array}{l}\text { Non } \\
\text { carrier }\end{array}$ & $\begin{array}{l}\text { Maternal } \\
\text { parents }\end{array}$ & M & 12 \\
\hline
\end{tabular}

(\# Mendelian Inheritance in Men); MI= mode of inheritance; $\mathrm{AR}=$ autosomal recessive; $\mathrm{AD}=$ autosomal dominant; $\mathrm{XLD}=\mathrm{X}$-linked dominant; $\mathrm{M}=$ male; $\mathrm{F}=$ female. 


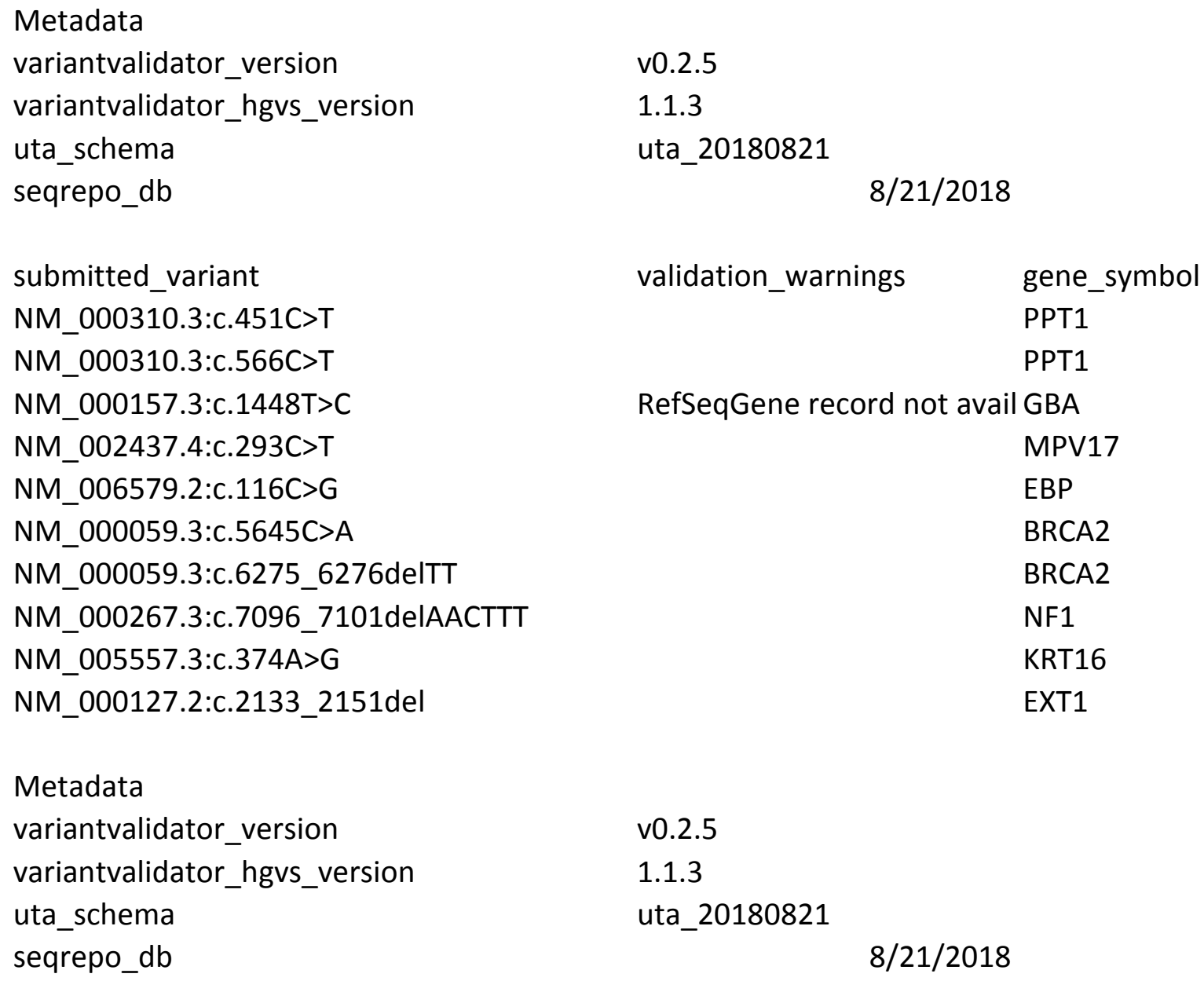

v0.2.5

1.1.3

uta_20180821

$8 / 21 / 2018$

v0.2.5

1.1.3

uta_20180821 


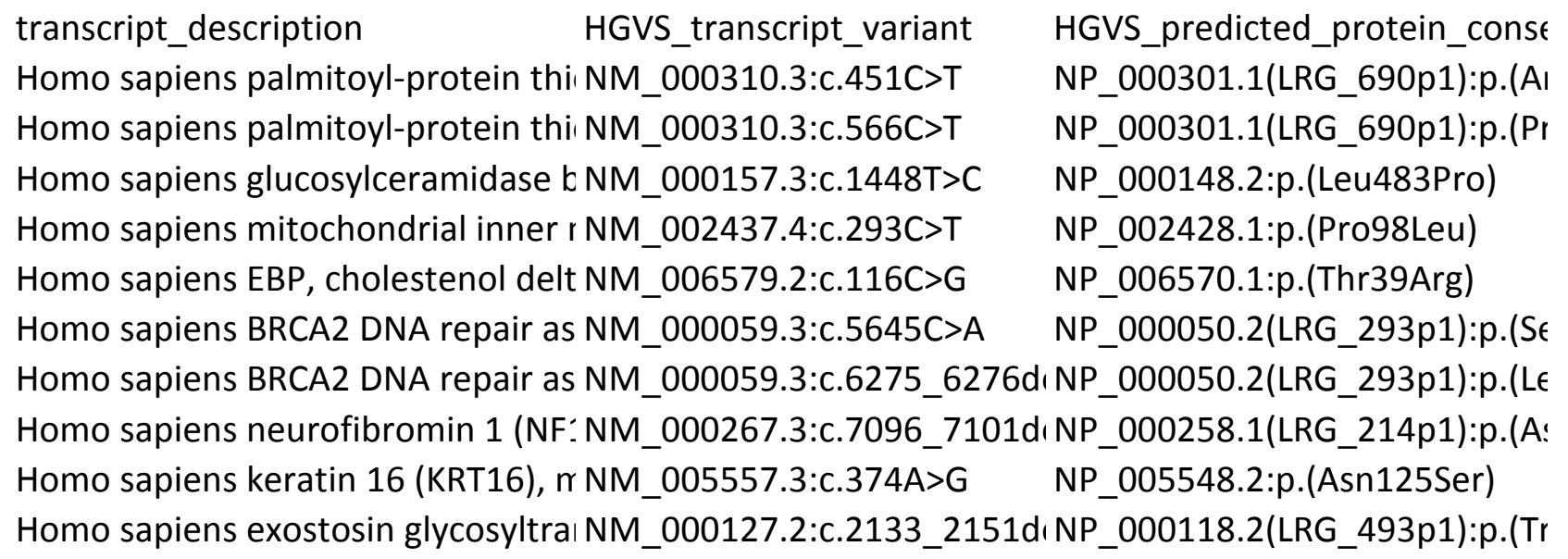


HGVS_predicted_protein_c(HGVS_Genon GRCh37_CHR GRCh37_POS GRCh37_ID

$\begin{array}{llrr}\text { NP_000301.1:p.(R151*) } & \text { NC_000001.1 } & 1 & 40555167 . \\ \text { NP_000301.1:p.(P189L) } & \text { NC_000001.1 } & 1 & 40546130 . \\ \text { NP_000148.2:p.(L483P) } & \text { NC_000001.1 } & 1 & 155205043 . \\ \text { NP_002428.1:p.(P98L) } & \text { NC_000002.1 } & 2 & 27535443 . \\ \text { NP_006570.1:p.(T39R) } & \text { NC_000023.1X } & & 48382275 . \\ \text { NP_000050.2:p.(S1882*) } & \text { NC_000013.1 } & 13 & 32914137 . \\ \text { NP_000050.2:p.(L2092Pfs*- NC_000013.1 } & 13 & 32914766 . \\ \text { NP_000258.1:p.(N2366_F2ミNC_000017.1 } & 17 & 29670115 . \\ \text { NP_005548.2:p.(N125S) } & \text { NC_000017.1 } & 17 & 39768567 . \\ \text { NP_000118.2:p.(W711*) } & \text { NC_000008.1 } & 8 & 118812040 .\end{array}$




$\begin{array}{ll}\text { GRCh37_REF } & \text { GRCh37_ALT } \\ \text { G } & \text { A } \\ \text { G } & \text { A } \\ \text { A } & \text { G } \\ \text { G } & \text { A } \\ \text { C } & \text { G } \\ \text { C } & \text { A } \\ \text { CTT } & \text { C } \\ \text { CTAACTT } & \mathrm{C} \\ \text { T } & \text { C } \\ \text { TCAGCGGCATGTAGCC T }\end{array}$

HGVS_Genomic_Description_GRCh38

NC_000001.11:g.40089495G $>A$

NC_000001.11:g.40080458G>A

NC_000001.11:g.155235252A>G

NC_000002.12:g.27312576G>A

NC_000023.11:g.48523887C>G

NC_000013.11:g.32340000C $>A$

NC_000013.11:g.32340630_32340631del

NC_000017.11:g.31343105_31343110del

NC_000017.11:g.41612315T>C

NC_000008.11:g.117799806_117799824del 


\begin{tabular}{|c|c|c|c|c|c|}
\hline \multirow[t]{5}{*}{ GRCh38_CHR } & \multicolumn{2}{|c|}{ GRCh38_POS } & \multirow[t]{2}{*}{ GRCh38_ID } & \multicolumn{2}{|c|}{ GRCh38_REF GRCh38_ALT } \\
\hline & 1 & 40089495 & & G & A \\
\hline & 1 & 40080458 & & G & A \\
\hline & 1 & 155235252 & & A & G \\
\hline & 2 & 27312576 & & G & A \\
\hline \multirow[t]{6}{*}{$\mathrm{x}$} & & 48523887 & & c & G \\
\hline & 13 & 32340000 & & c & A \\
\hline & 13 & 32340629 & & CTT & C \\
\hline & 17 & 31343097 & & СТАACTT & c \\
\hline & 17 & 41612315 & & $\mathrm{~T}$ & c \\
\hline & 8 & 117799801 & & TCAGCGG & \\
\hline
\end{tabular}


RefSeqGene_genome_con 1 HGVS_LRG_viHGVS_LRG_tr HGVS_RefSec alt_genomic_loci

$$
\begin{array}{r}
\text { LRG_690:g.12LRG_690t1:c. NG_009192.1:g.12976C>T } \\
\text { LRG_690:g.2zLRG_690t1:c. NG_009192.1:g.22013C>T } \\
\text { NW_003315906.1:g.40275/ } \\
\text { NG_008075.1:g.14989C>T } \\
\text { NG_007452.1 NW_004070880.2:g.76331t } \\
\text { LRG_293:g.2c LRG_293t1:c. NG_012772.3:g.29521C>A } \\
\text { LRG_293:g.3C LRG_293t1:c. NG_012772.3:g.30151_30152del } \\
\text { LRG_214:g.25LRG_214t1:c. NG_009018.1:g.253129_253134del } \\
\text { NG_008301.1:g.5513A>G } \\
\text { LRG_493:g.31LRG_493t1:c. NG_007455.2:g.317000_317018del }
\end{array}
$$


A>G;grch37;HSCHR1_2_CTG31-40275-A-G|NW_003315906.1:g.40275A>G;grch38;HSCHR1_2_CT jC>G;grch37;HG1436_HG1432_PATCH-763316-C-G 
G31-40275-A-G 


\section{Supplementary Materials and Methods}

\section{Sample collection and processing}

Genomic DNAs from family members were extracted from blood following standard procedures. Briefly, $8 \mathrm{~mL}$ of maternal peripheral blood was collected in cell-free DNA BCT tubes (Streck, Omaha, NE, USA) or cell-free DNA collection tubes (Roche Diagnostics,

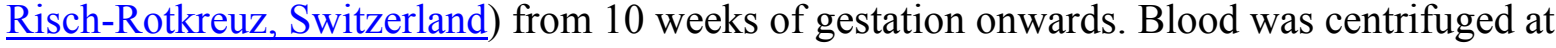
$1600 \mathrm{~g}$ for $10 \mathrm{~min}$ at $4^{\circ} \mathrm{C}$, and the plasma portion was carefully removed and centrifuged again at $16,000 \mathrm{~g}$ for $10 \mathrm{~min}$. The plasma $(4 \mathrm{ml})$ and buffy coat were stored separately. DNA from the buffy coat was extracted following the "Blood and Body fluid" protocol of the QIAamp DSP DNA Blood Mini Kit (Qiagen, Hilden, Germany). cfDNA was extracted from 4 mL plasma automatically, using the Maxwell HT cfDNA kit (Promega, Wisconsin, USA) on the Hamilton Liquid Handler according to the manufacturer's recommendations.

\section{Parental data phasing}

The biallelic SNP sites from parental genotypes were extracted with lower coverage threshold of 10-fold and upper threshold of 500-fold. The parental genotypes are divided into 5 groups according to paternal and maternal allele combinations. For illustration, the biallelic sites are shown as carrying "A" or "B" allele.

\begin{tabular}{|l|c|c|}
\hline Groups & Paternal genotype & Maternal genotype \\
\hline Type 1 & $\mathrm{~A} / \mathrm{A}$ & $\mathrm{B} / \mathrm{B}$ \\
\hline Type 2 (Paternal) & $\mathrm{A} / \mathrm{B}$ & $\mathrm{A} / \mathrm{A}$ \\
\hline & $\mathrm{A} / \mathrm{B}$ & $\mathrm{B} / \mathrm{B}$ \\
\hline Type 3 (Maternal) & $\mathrm{A} / \mathrm{A}$ & $\mathrm{A} / \mathrm{B}$ \\
\hline & $\mathrm{B} / \mathrm{B}$ & $\mathrm{A} / \mathrm{B}$ \\
\hline Type 4 & $\mathrm{~A} / \mathrm{B}$ & $\mathrm{A} / \mathrm{B}$ \\
\hline Type 5 & $\mathrm{B} / \mathrm{B}$ & $\mathrm{B} / \mathrm{B}$ \\
\hline
\end{tabular}

Fetal fraction estimation and error estimation 
At each Type 1 SNP site, where maternal alleles are different from paternal alleles, the paternal specific allele can be identified in maternal plasma unambiguously. By counting reads that contain paternal specific alleles $(f)$ and total number of reads $(N)$ in such SNP sites, we estimated the fetal fraction $(\mathrm{FF})$ percentage as:

$$
F F=\frac{2 * f}{N}
$$

Technical and biological factors contribute to inconsistent estimation of FF from site to site. Considering the variability and potential copy number changes, all paternal specific alleles were sum and divided by total reads across all Type 1 SNP loci to give FF estimation; median of FF estimation on all Type 1 SNP sites was also calculated to ensure justified estimation of FF.

Sequencing and alignment errors in maternal plasma were estimated by Type 5 SNPs. When both parents have the same genotypes (alternative alleles), the fetus is supposed to have the same genotypes, unless a de novo mutation happens, which is of low probability. We counted the number of reference alleles and total alleles present on Type 5 SNP sites. The ratio of reference allele numbers to total numbers were used to approximate errors (Supplementary Table S2).

\section{Fetal haplotype inference based on fetal allele ratio (FAR)}

The presence of fetal allele in maternal plasma was measured using the following metric fetal allele ratio (FAR)

$$
F A R_{i}=\left\{\begin{array}{c}
\frac{2 * f_{i}}{N_{i}}, \text { SNP site } i \text { is Type } 2 \text { SNP } \\
\frac{M H 1_{i}-M H 2_{i}}{M H 1_{i}+M H 2_{i}}, \text { SNP site } i \text { is Type } 3 \text { SNP }
\end{array}\right.
$$

FAR calculations combined with subcategory classifications estimate fetal genome-wide 
haplotype. FAR value changes in subcategory indicate the homolog changes in inheritance, and thus perform phasing of recombinant haplotypes. In paternal transmission, we flipped the FAR value of P1 category by using 0-FAR for visualization purpose. Assuming 100-fold coverage and $10 \% \mathrm{FF}$, when paternal homolog $\mathrm{H} 1$ is inherited, FAR of P1 SNPs will be 0 and FAR of P2 will be 10\%, as shown in the example Figure 1b, and when paternal homolog $\mathrm{H} 2$ is inherited, FAR of P1 SNPs will be $-10 \%$ and FAR of $\mathrm{P} 2$ will be 0 . In maternal transmission, FAR of M1 SNPs will be $10 \%$ and FAR of M2 will be 0 when maternal homolog H1 is inherited, and FAR of M1 SNPs will be 0 and FAR of M2 will be $-10 \%$ when maternal homolog $\mathrm{H} 2$ is inherited. FAR values of subcategories (P1 and P2 or M1 and M1) hence infer haplotype results reciprocally.

While FAR provides information on the allele dosage of the fetus and site-by-site genotype of informative SNPs could be predicted, there is high variability in practical estimation due to technical bias, biological features of plasma DNA, and dominant maternal DNA in maternal plasma. To overcome the noises in site-by-site FAR estimations, we employed circular binary segmentation $^{1}(\mathrm{CBS})$ to estimate fetal allele ratio in contiguous regions and to identify likely recombination sites. Briefly, $F A R_{1}, \ldots, F A R_{i}$ raw values were calculated for Type 2 or Type 3 sub-categorical SNPs, and the data was smoothed by adjusting outlier values, resulting in $\widetilde{F A R}_{1}, \ldots, \widetilde{F A R}_{l}$. The likelihood ratio test was then performed on smoothed data to test the null hypothesis of no change-point in the data. If the test statistic exceeds threshold and reject the null hypothesis, the change points were identified. By detecting these change points in fetal allele ratio values, which correspond to homolog inheritance changes, we deduce the possible genomic locations of switchovers. The haplotype blocks derived from two subcategories were screened after the segmentation to avoid long regions that contains no informative SNPs. Within one block, if the genomic distance between two adjacent 
informative SNPs were greater than $3 \mathrm{Mb}$, the block was divided into two blocks with the two end positions (end of block 1 and start of block 2) corresponding to the two SNPs genomic positions. The segmental mean FAR estimates from subcategory P1 and P2 or M1 and M2 were examined to check whether both subcategories segmentation reach the same conclusion. We concluded that paternal $\mathrm{H} 1$ was inherited only when $\overline{F A R_{P 1}}$ was in the range $(-0.25 *$ $F F, 0.25 * F F)$ and $\overline{F A R_{P 2}}$ was in the range $(0.75 * F F, 1.25 * F F)$, and paternal $\mathrm{H} 2$ was inherited when when $\overline{F A R_{P 1}}$ was within $(-1.25 * F F,-0.75 * F F)$ and $\overline{F A R_{P 2}}$ was within $(-0.25 * F F, 0.25 * F F)$. The same FAR value thresholds applied to maternal $\mathrm{H} 1$ and $\mathrm{H} 2$ inheritance. Fetal allele ratio value that fell out of threshold range was defined as inconclusive result.

\section{Newborn haplotype}

The newborn child's sequencing data was analyzed in conjunction with the parental genotypes of the family. Genotypes of the family trio were called together with HaplotypeCaller. We matched the child genotypes with phased parental genomes that were resolved using affected offspring or parents of the couple. Then parental homologous that defined by phasing with relatives were assigned to matched child genotypes site by site. SNPs with unambiguously assigned homolog 1 or homolog 2 were used to infer child haplotype. In cases where parental haplotypes were defined by the affected offspring, there might be small recombinations shown on child haplotype due to the crossovers from the sibling and the child happen in close genomic locations.

Accuracy compared with born child haplotypes were measured in haplotype block length and informative SNPs inference. Haplotype accuracy was calculated as the percentage of matched haplotype blocks length divided by the total length of haplotypes obtained in born child 
haplotypes. Informative SNPs inference accuracy was measured by matching correctly assigned SNP genotypes in cfDNA using haplotypes information to born child genotypes.

\section{Reference bias correction}

We observed bias towards reference allele at heterozygous sites in cfDNA samples.

Supplementary Figure S11 showed the reference allele frequency of heterozygous sites in multiple cfDNA samples, where both maternal and fetal SNPs were assumed to be heterozygous with haplotype information available. For M1 subcategory, when the fetus inherits maternal $\mathrm{H} 2$ from mother, the fetus genotypes are heterozygous in the SNP sites within the haplotype block. For M2 subcategory, the fetus genotypes are heterozygous when maternal $\mathrm{H} 1$ is inherited from the mother. By computing reference allele frequency of corresponding heterozygous sites for M1 and M2 separately, the allele counts displayed bias towards reference allele. Though there might be less bias towards reference allele at SNPs where the genotype of the mother is heterozygous and the fetus is homozygous, the dominant maternal alleles skews the allele ratio. Maternal haplotyping inference can be distorted due to such bias. We performed bias correction by imposing a correction factor to reference and alternative alleles count in plasma samples to reduce bias. We used an arbitrary correction factor of 0.525 to estimate putative haplotype inheritance. Additionally, from estimated haplotype we sampled SNPs to approximate true reference allele bias per chromosome and per subcategory using maximum likelihood estimation. The computed correction factors were applied to the reference and alternative allele counts to adjust value of FAR for each SNP.

\section{Fetal fraction simulation and cfDNA downsampling}

We mixed different proportions of reads computationally from the trisomy family where the mother and the child genomic DNA sequencing were available. Samtools v1.3.1 ${ }^{2}$ were used 
to randomly subsample reads from bam files and in total about 70 million reads $(\sim 85 \mathrm{x}$ on target coverage) were sampled from the mother and the child. By mixing all reads from mother and $4 \%$ of reads from the child, FF of $5.5 \%$ was simulated. To evaluate lower FF limit for paternal inheritance haplotyping, all reads from mother and less than $4 \%$ of reads from the child were mixed, though the total number of reads decreased slightly in this case. FF were estimated using mixture proportions. For each sampling proportion pairs, we repeated for 10 times with different sampling seeds. The child haplotype was phased directly from family trio analysis using GATK PhaseByTransmission. Haplotyping results from in silico simulation samples were compared to the reference child haplotype to measure genome-wide accuracy and crossover gaps. Chromosome 21 were excluded from accuracy benchmarking.

We downsampled the cfDNA sample to different proportions using samtools. Each subsampling proportion was repeated for 10 times using different seeds. Downsampling was conducted until only 11 million reads were left. We again measured the accuracies against PGT embryo haplotying result and crossover resolutions for all subsampling levels.

\section{Integration of Type 4 SNPs in cfDNA haplotyping analysis}

In cases where offspring (sibling of the born child) genotype data were available, we used Type 4 SNPs to improve haplotyping accuracy. Type 4 SNPs are defined as heterozygous SNPs for both parents and these SNPs can be phased (i.e. the genotype of sibling on these SNPs sites are homozygous). We first inferred paternal haplotyping with Type 2 SNPs. Using paternal haplotype information, we could deduce the allele that fetus inherited from a Type 4 SNP when the site was within resolved haplotype block, and thus the site became unambiguous. We then regarded the Type 4 site as a homozygous site and turned the site into 
a Type 3 SNP site, as the paternal genotype was viewed as homozygous and maternal genotype is heterozygous. We further integrated unambiguous Type 4 SNPs into Type 3 SNPs and performed same analysis as when we only use Type 3 SNPs.

\section{Aneuploidy detection}

Genomic DNA from all the family members (father, mother, unaffected sibling, and affected proband) was extracted following standard procedures. Parental genotype phasing was done using an unaffected offspring from the family, and haplotype inheritance was deduced from the spike-in DNA samples. Chromosomal abnormalities result in FAR value deviation from the expected FF. The shifting pattern is associated with FF. In maternal meiotic trisomy, the overrepresented allele leads maternal FAR value to $\pm \frac{1}{2} F F /\left(1+\frac{1}{2} F F\right)$, while in mitotic

trisomy, M1 FAR drifts to $\pm \frac{3}{2} F F /\left(1+\frac{1}{2} F F\right)$ and M2 FAR to $\pm \frac{1}{2} F F /\left(1+\frac{1}{2} F F\right)$. Paternal FAR value deviates from normal FF by $\pm F F^{2} /(2+F F)$, which is of very low amount to be distinguished from normal disomy cases.

\section{Aneuploidy confirmation in the trisomy child and maternal copy number}

As the trisomy child is a male, we used fifteen normal male gDNA samples from our clinical dataset to build a reference set. The normalized coverage per target was calculated by total coverage within the target divided by the target region size. We calculated GC content of each target and used Loess regression to correct GC bias. A further step of normalization was carried out by dividing the GC corrected median target coverage count. The reference set summarized the average normalized count and standard deviation across the 15 samples. Log2 ratio per target was computed using normalized count from the trisomy child gDNA sample against the corresponding normalized count in the reference set. We used CBS 
segmentation on $\log 2$ ratios to detect copy number change as previously described. To infer homologous recombination sites and origin of aneuploidy on chromosome 21 , we analyzed the family data using the child reference allele frequency count. Maternal copy number detection of family 9_074 used same approach as the aneuploidy detection, except that for cfDNA maternal copy number, a reference was created by 8 cfDNA from other families.

\section{Supplementary References}

1. Shen, J. J. \& Zhang, N. R. Change-point model on nonhomogeneous Poisson processes with application in copy number profiling by next-generation DNA sequencing. Ann. Appl. Stat. 6, 476-496 (2012).

2. Li, H. et al. The Sequence Alignment/Map format and SAMtools. Bioinformatics 25, 20782079 (2009). 


\section{Supplementary Figure Legends}

\section{Supplementary Figure S1. Study workflow.}

\section{Supplementary Figure S2. Scheme of parental genotype phasing via parents of the}

couple. a, Paternal haplotype derived from paternal parents with the mutation inherited from grandmother. b, Maternal haplotype derived from maternal parents with the mutation inherited from grandmother.

\section{Supplementary Figure S3. cffDNA haplotyping profiles with different phasing options.} Chromosome-specific cffDNA haplotyping plots are depicted for three phasing options. In a, a family with an affected child were used to deduce paternal (upper panel) and maternal haplotype (lower panel) inheritance. The first track shows raw fetal allele ratio (FAR) values, and each dot represents an informative SNP, with red and blue indicating paternal P1 and P2 SNPs subcategories, respectively. We flipped FAR for P1 subcategory to negative values for better visualization. The same red and blue color pattern was also applied to distinguish maternal M1 and M2 SNPs subcategories. The second track corresponds to FAR segmentation, where the red dotted line represents segmented P1 or M1 FAR and blue for segmented P2 or M2 FAR, and the distance between P1 and P2 or M1 and M2 segmentation in the same genomic region indicates fetal. The third track is the deduced fetal haplotypes inherited from father or mother. Dark and light blue denotes inheritance of homologue $1(\mathrm{H} 1)$ and homologue $2(\mathrm{H} 2)$ from the father, respectively. In the same way, dark and light red denotes inheritance of homologue $1(\mathrm{H} 1)$ and homologue $2(\mathrm{H} 2)$ from the mother, respectively. Switch between $\mathrm{H} 1$ and $\mathrm{H} 2$ denotes homologues recombination sites, which are also corroborated by the segmented FAR profiles. Centromere and regions with inconclusive 
haplotype results are shown as white gap in the final haplotype result. b. Parents of the father are available for phasing and only paternal haplotype inheritance is shown. c, Parents of the mother are available for phasing and only maternal haplotype inheritance is shown.

\section{Supplementary Figure S4. cffDNA haplotyping for monogenic disorders with phasing}

from parents of the couple. a-d, Families with parents of the father available for phasing. ab, haplotypes inferred from cfDNA were compared to both neonatal and embryo haplotypes. Dark blue represents the homologue inherited from the grandfather of the child and light blue represent the homologue inherited from the grandmother. In Family 7 (d), the father inherited the mutant allele from the grandfather and the disease locus crosses the light blue haplotype block indicating the wild-type allele from grandmother was transmitted. e-f, Families with parents of the mother for phasing, dark red represent the homologue inherited from the grandfather, and light red represent the homologue inherited from the grandmother. In both families, the mutant allele was inherited from the grandmother.

\section{Supplementary Figure S5. Factors that impact the accuracy cffDNA haplotyping.} Lower informative SNPs inference accuracy near homologous recombination-sites. a, Measurement of accuracy for cfDNA haplotyping and illustration of density of SNPs, sequencing depth and FF effects on haplotype accuracy and crossover resolution metrics. b, For a $9.5 \% \mathrm{FF}$ cfDNA samples, homologous recombination accuracy measured in $10 \mathrm{~Kb}$ bins that flank out from both sides of true (neonatal) crossovers, is shown. On x-axis, 0 denotes the true crossover breakpoint sites. Positive and negative distances correspond to extension to the right and to the left side of breakpoints. c, Homologous recombination accuracy measure in terms of haplotype-based informative SNPs. Informative SNPs genotypes inferred from cffDNA haplotyping were evaluated against neonatal genotypes from Family 1_181. x-axis 
denotes called informative SNPs in the cfDNA samples and 0 indicates neonatal crossover breakpoint sites. Extending from crossovers, accuracy of informative SNPs to the left (negative values) and to the right side (positive) was measured for maternal and paternal transmissions.

\section{Supplementary Figure S6. Genome-wide haplotyping comparison with reference} haplotype. a, cffDNA haplotyping results compared to born child haplotype. b, cffDNA haplotyping results compared to embryo single-cell haplotype. In all sub figures, paternal inheritance is shown in dark and light blue, and maternal inheritance is shown in dark and light red. Upper tracks are reference (born child/embryo) haplotyping results and lower tracks are cffDNA haplotyping results.

\section{Supplementary Figure S7. Additional informative SNPs improve performance of} cffDNA haplarithmisis. Integrating phased type 4 SNPs into maternal informative SNPs show higher accuracy and decreased crossover gaps in cffDNA haplotyping at varying simulated fetal fraction levels.

Supplementary Figure S8. cffDNA haplotyping accuracy of downsampled data. Effect of sequencing depth on the performance of cffDNA haplotyping for a 9.5\% FF sample.

Supplementary Figure S9. Patterns of chromosomal anomalies. Assuming 100-fold coverage and $10 \%$ fetal fraction, different segmentation schemes are shown in normal, 
maternal trisomy, and paternal trisomy in a and corresponding FAR values are displayed in b.

\section{Supplementary Figure S10. Spike-in samples FAR values deviated from normal values} significantly. In the $20 \%$ spike-in sample, a, For M1 maternal informative SNPs, analysis by unpaired two groups $t$-test of FAR values of chromosome 21 and FAR values of other chromosomes indicating inheritance of maternal H1 showed significant ( $p$-value $<2.22 \mathrm{e}-16$ ) difference in mean of FAR values. b, For M2 maternal informative SNPs, $t$-test also showed significant (p-value $<2.22 \mathrm{e}-16$ ) difference between mean of chromosome 21 FAR values and other chromosomes FAR values of maternal H1 inheritance. In the $10 \%$ spike-in sample, $t$ test showed significant FAR values shifting for M1 maternal informative SNPS shown in $\mathbf{c}$ $(\mathrm{p}$-value $=1.5 \mathrm{e}-10)$ and for M2 maternal informative SNPs shown in $\mathbf{d}(\mathrm{p}$-value $=2.9 \mathrm{e}-15)$, respectively.

Supplementary Figure S11. Simulation of lower fetal fraction effect on paternal haplotyping accuracy. Genome-wide paternal inheritance haplotyping accuracy and crossover resolution of lower fetal fraction.

Supplementary Figure S12. Maternal segmental duplication. a, $\log 2$ ratio profile showing $\sim 1.5 \mathrm{Mb}$ duplication from maternal gDNA in family 9_074. $\mathbf{b}, \log 2$ ratio profile of maternal copy number presence in cfDNA sample of case 9_074.

Supplementary Figure S13. Reference allele bias in cfDNA samples. Density plot of reference allele frequencies at heterozygous SNPs (both maternal and fetal) for 6 cfDNA 
samples. The distribution show centered reference allele frequency to be higher than theoretical value of 0.5 . 


\section{Supplementary Tables}

Supplementary Table S1. cffDNA haplotyping accuracy

\begin{tabular}{|c|c|c|c|c|c|c|c|c|c|c|c|c|}
\hline \multirow[t]{2}{*}{ Family } & \multirow[t]{2}{*}{$\begin{array}{c}\text { Plasma } \\
\text { DNA } \\
\text { Coverage }^{1}\end{array}$} & \multirow[t]{2}{*}{$\begin{array}{c}\text { Fetal } \\
\text { Fraction }^{2}\end{array}$} & \multicolumn{2}{|c|}{ No. SNPs } & \multicolumn{2}{|c|}{$\begin{array}{c}\text { Haplotype accuracy } \\
(\%) \text { w.r.t. } \\
\text { neonatal haplotype }\end{array}$} & \multicolumn{2}{|c|}{$\begin{array}{c}\text { Informative SNPs } \\
\text { accuracy (\%) w.r.t } \\
\text { neonatal SNPs }\end{array}$} & \multicolumn{2}{|c|}{$\begin{array}{c}\text { Haplotype accuracy (\%) } \\
\text { w.r.t. } \\
\text { embryo haplotype } \\
\end{array}$} & \multicolumn{2}{|c|}{$\begin{array}{l}\text { Crossover resolution } \\
\qquad(\mathrm{Kb})\end{array}$} \\
\hline & & & Paternal & Maternal & Paternal & Maternal & Paternal & Maternal & Paternal & Maternal & Paternal & Maternal \\
\hline 1_181 & $76 x$ & $9.5 \%$ & 34,043 & 34,362 & 98.94 & 94.53 & 99.70 & 95.64 & 99.31 & 95.69 & 268.93 & 818.25 \\
\hline $2 \_186^{3}$ & $85 x$ & $9.8 \%$ & 36,579 & 39,224 & - & - & - & - & 99.05 & 93.16 & 149.09 & 570.46 \\
\hline $3 \_085$ & $77 x$ & $16.5 \%$ & 38,573 & 40,887 & - & - & - & - & 99.42 & 97.24 & 131.18 & 433.74 \\
\hline 4_158 & $64 x$ & $8.2 \%$ & 37,855 & - & 98.84 & - & 99.04 & - & 98.86 & - & 308.47 & - \\
\hline 5_149 & $96 x$ & $6.8 \%$ & 40,695 & - & - & - & - & - & 99.77 & - & 148.52 & - \\
\hline 6_150 & $63 x$ & $6.2 \%$ & 39,237 & - & 99.00 & - & 99.40 & - & 99.15 & - & 330.53 & - \\
\hline 7_054 & $50 \mathrm{x}$ & $13.4 \%$ & 36,558 & - & - & - & - & - & 99.46 & - & 162.57 & - \\
\hline $8 \_078$ & $82 x$ & $11.4 \%$ & - & 43,401 & - & - & - & - & - & 92.68 & - & 1619.10 \\
\hline $9 \_074$ & $82 x$ & $8.4 \%$ & - & 40,651 & - & - & - & - & - & 89.41 & - & 1686.04 \\
\hline
\end{tabular}

${ }^{1}$ Median on-target de-duplicated fragment coverage; ${ }^{2}$ Estimated from Type $1 \mathrm{SNPs} ;{ }^{3}$ Family with consanguinity 
Supplementary Table S2. Sequencing or mapping error rate in cfDNA and Spike-in samples

\begin{tabular}{lr}
\hline Sample & Error rate (\%) \\
\hline 1_181 & 0.0430 \\
2_186 & 0.0498 \\
3_085 & 0.0498 \\
4_158 & 0.0387 \\
5_149 & 0.0563 \\
6_150 & 0.0465 \\
7_054 & 0.1025 \\
8_078 & 0.1439 \\
9_074 & 0.1196 \\
Spike-in_20\% & 0.0437 \\
Spike-in_10\% & 0.0445 \\
\hline
\end{tabular}




\section{LICENCE TO PUBLISH}

Manuscript Number:

\section{GIM-D-19-00723}

Proposed Title of the Article:

Journal Name:

Author(s) [Please list all authors, continuing on a separate sheet if necessary]:

Huiwen Che, Darine Villela, Eftychia Dimitriadou, Cindy Melotte, Nathalie Brison, Maria Neofytou, Kris Van (the "Author(s)")

Den Boaaert Olaa Tsuiko Koen Devriendt Fric Leaius Masoud Zamani Esteki_Thierrv Voet_loris Rohert

\author{
(the "Journal")
}

Miscellaneous [for office use only]

will consider publishing this article, including any supplementary information and graphic elements therein (e.g. illustrations, charts, moving images) (the 'Article'). Headings are for convenience only.

\section{Grant of Rights}

In consideration of the Licensee evaluating the Article for publication, the Author(s) grant the Licensee the exclusive (except as set out in clauses 3;4 and 5a) iv) and sub-licensable right, unlimited in time and territory, to copy-edit, reproduce, publish, distribute, transmit, make available and store the Article, including abstracts thereof, in all forms of media of expression now known or developed in the future, including pre- and reprints, translations, photographic reproductions and extensions.

Furthermore, to enable additional publishing services, such as promotion of the Article, the Author(s) grant the Licensee the right to use the Article (including the use of any graphic elements on a stand-alone basis) in whole or in part in electronic form, such as for display in databases or data networks (e.g. the Internet), or for print or download to stationary or portable devices. This includes interactive and multimedia use as well as posting the Article in full or in part or its abstract on social media, and the right to alter the Article to the extent necessary for such use. The Licensee may also let third parties share the Article in full or in part or its abstract on social media and may in this context sub-license the Article and its abstract to social media users. Author(s) grant to Licensee the right to re-license Article metadata without restriction (including but not limited to author name, title, abstract, citation, references, keyword and any additional information as determined by Licensee).

\section{Self-Archiving}

Author(s) are permitted to self-archive a pre-print and an Author's accepted manuscript version of their Article.

a) A pre-print is the Author's version of the Article before peer-review has taken place ("Pre-Print"). Prior to acceptance for publication, Author(s) retain the right to make a Pre-Print of their Article available on any of the following: their own personal, self-maintained website; a legally compliant pre-print server such as but not limited to arXiv and bioRxiv. Once the Article has been published, the Author(s) should update the acknowledgement and provide a link to the definitive version on the publisher's website: "This is a pre-print of an article published in [insert journal title]. The final authenticated version is available online at: https://doi.org/[insert DOI]"

b) An Author's Accepted Manuscript ("AAM") is the version accepted for publication in a journal following peer review but prior to copyediting and typesetting that can be made available under the following conditions:

Author(s) retain the right to make an AAM of their Article available for public release on any of the following 6 months after first publication ("Embargo Period"): their own personal, self-maintained website; their employer's internal website; their institutional and/or funder repositories. AAMs may also be deposited in such repositories immediately on acceptance, provided that they are not made publicly available until after the Embargo Period.

An acknowledgement in the following form should be included, together with a link to the published version on the publisher's website: "This is a post-peer-review, pre-copyedit version of an article published in [insert journal title]. The final authenticated version is available online at: $h$ ttp://dx.doi.org/[insert DOI]"

4. Author's Retained Rights

Author(s) retain the following non-exclusive rights for the published version provided that, when reproducing the Article or extracts from it, the Author(s) acknowledge and reference first publication in the Journal:

a) to reuse graphic elements created by the Author(s) and contained in the Article, in

presentations and other works created by them

b) they and any academic institution where they work at the time may reproduce the
Article for the purpose of course teaching (but not for inclusion in course pack material for onward sale by libraries and institutions); and

c) to reproduce, or to allow a third party Licensee to reproduce the Article in whole or in part in any thesis written by the Author(s).

d) to reproduce, or to allow a third party Licensee to reproduce the Article, in whole or in part, in any other type of work (other than thesis) written by the Author(s) for distribution by a publisher after an embargo period of 12 months.

\section{Warranties}

The Author(s) warrant and represent that:

a) (i) the Author(s) are the sole copyright owners or have been authorised by any additional copyright owner to grant the rights defined in clause 2, (ii) the Article does not infringe any intellectual property rights (including without limitation copyright, database rights or trade mark rights) or other third party rights and no licence from or payments to a third party are required to publish the Article, (iii) the Article has not been previously published or licensed, (iv) if the Article contains materials from other sources (e.g. illustrations, tables, text quotations), Author(s) have obtained written permissions to the extent necessary from the copyright holder(s), to license to the Licensee the same rights as set out in clause 2 but on a non-exclusive basis and without the right to use any graphic elements on a stand-alone basis and have cited any such materials correctly:

b) all of the facts contained in the Article are according to the current body of science true and accurate;

c) nothing in the Article is obscene, defamatory, violates any right of privacy or publicity, infringes any other human, personal or other rights of any person or entity or is otherwise unlawful and that informed consent to publish has been obtained for all research participants;

d) nothing in the Article infringes any duty of confidentiality which any of the Author(s) might owe to anyone else or violates any contract, express or implied, of any of the Author(s). All of the institutions in which work recorded in the Article was created or carried out have authorised and approved such research and publication; and e) the signatory (the Author or the employer) who has signed this agreement has full right, power and authority to enter into this agreement on behalf of all of the Author(s)

\section{Cooperation}

a) The Author(s) shall cooperate fully with the Licensee in relation to any legal action that might arise from the publication of the Article and the Author(s) shall give the Licensee access at reasonable times to any relevant accounts, documents and records within the power or control of the Author(s). The Author(s) agree that the distributing entity is intended to have the benefit of and shall have the right to enforce the terms of this agreement.

b) The Author(s) authorise the Licensee to take such steps as it considers necessary at its own expense in the Author(s)' name and on their behalf if the Licensee believes that a third party is infringing or is likely to infringe copyright in the Article including but not limited to initiating legal proceedings.

7. Author List

After signing, changes of authorship or the order of the authors listed will not be accepted unless formally approved in writing by the Licensee.

\section{Edits \& Corrections}

The Author(s) agree(s) that the Licensee may retract the Article or publish a correction or other notice in relation to the Article if the Licensee considers in its reasonable opinion that such actions are appropriate from a legal, editorial or research integrity perspective.
Signed for and on behalf of the Author(s):

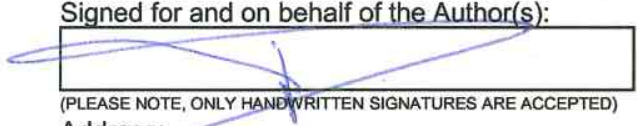

SIGNATURES ARE ACCEPTED)

\section{Pinfluger: IOAIS VERMEESCH}

Date:

$19 / 11 / 2019$

Address:

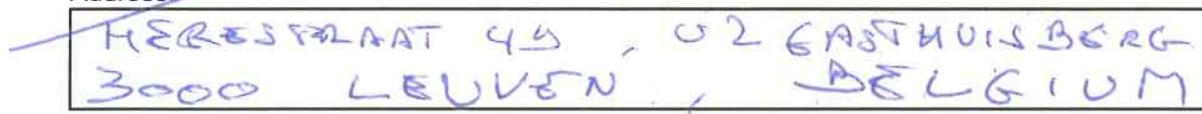


Corresponding Author Name: Joris Robert Vermeesch

Manuscript Number: GIM-D-19-00723

Reporting Checklist* (Please see page 3 for instructions on uploading this file)

\section{Genetics inMedicine}

This checklist is used to ensure good reporting standards and to improve the reproducibility of published results. Please respond completely to all questions relevant to your manuscript. For sections that are not applicable please fill in NA. For more information, please read the journal's Guide to Authors.

Check here to confirm that the following information is available in the Material \& Methods section:

- the exact sample size $(n)$ for each experimental group/condition, given as a number, not a range;

- a description of the sample collection allowing the reader to understand whether the samples represent technical or biological replicates (including how many animals, litters, culture, etc.);

- a statement of how many times the experiment shown was replicated in the laboratory;

- definitions of statistical methods and measures: (For small sample sizes $(n<5)$ descriptive statistics are not appropriate, instead plot individual data points)

$\circ \quad$ very common tests, such as $t$-test, simple $\chi^{2}$ tests, Wilcoxon and Mann-Whitney tests, can be unambiguously identified by name only, but more complex techniques should be described in the methods section;

- are tests one-sided or two-sided?

$\circ$ are there adjustments for multiple comparisons?

- statistical test results, e.g., $P$ values;

- definition of 'center values' as median or mean;

- definition of error bars as s.d. or s.e.m. or c.i.

Please ensure that the answers to the following questions are reported both in the manuscript itself and in the space below. We encourage you to include a specific subsection in the methods section each for statistics, reagents and animal models. Below, provide the text as it appears in the manuscript as well as the page number.

\section{Statistics and general methods}

1. How was the sample size chosen to ensure adequate power to detect a pre-specified effect size? (Give text and page \#)

For animal studies, include a statement about sample size estimate even if no statistical methods were used.

2. Describe inclusion/exclusion criteria if samples or animals were excluded from the analysis. Were the criteria pre-established? (Give text and page \#)

3. If a method of randomization was used to determine how samples/animals were allocated to experimental groups and processed, describe it. (Give text and page \#)

For animal studies, include a statement about randomization even if no randomization was used.

\section{Text AND page number from manuscript}

Not available

Not available

Not available

Not available

Not available 
4. If the investigator was blinded to the group allocation during the experiment and/or when assessing the outcome, state the extent of blinding. (Give text and page \#)

For animal studies, include a statement about blinding even if no blinding was done.

5. For every figure, are statistical tests justified as appropriate?

Do the data meet the assumptions of the tests (e.g., normal distribution)?

Is there an estimate of variation within each group of data?

Is the variance similar between the groups that are being statistically compared? (Give text and page \#)

\section{Reagents}

6. Report the source of antibodies (vendor and catalog number)

7. Identify the source of cell lines and report if they were recently authenticated (e.g., by STR profiling) and tested for mycoplasma contamination

\section{Animal Models}

8. Report species, strain, sex and age of animals

9. For experiments involving live vertebrates, include a statement of compliance with ethical regulations and identify the committee(s) approving the experiments.
Not available

Not available

Yes

Not available

Not available

Not available

\section{Text AND page number from manuscript}

Not available

Not available

Text AND page number from manuscript

Not available

Not available

10. We recommend consulting the ARRIVE guidelines ( $\underline{\text { LLS Biol. 8(6), e1000412,2010) }}$ to ensure that other relevant aspects of animal studies are adequately reported. 


\section{Human subjects}

11. Identify the committee(s) approving the study protocol.

12. Include a statement confirming that informed consent was obtained from all subjects.

13. For publication of patient photos, include a statement confirming that consent to publish was obtained. For more information, please see http://www.icmje.org/recommendations/browse/r oles-and-responsibilities/protection-of-researchparticipants.html.

14. Report the clinical trial registration number (at ClinicalTrials.gov or equivalent).

\section{Text AND page number from manuscript}

Page6. This study was approved by the local Ethical Committees of the University Hospital Leuven (S59324).

Page6. Women with a successful pregnancy following PGT-M were recruited at the UZ Leuven Hospital, with informed

Not available

\begin{tabular}{|l|} 
\\
Not available \\
\hline Not available \\
\hline
\end{tabular}

15. For phase II and III randomized controlled trials, please refer to the CONSORT statement and submit the CONSORT checklist with your submission.

16. For tumor marker prognostic studies, we recommend that you follow the REMARK reporting guidelines.

\section{Data deposition}

17. Provide accession codes for deposited data. Data deposition in a public repository is recommended for:

a. Protein, DNA and RNA sequences

b. Macromolecular structures

c. Crystallographic data for small molecules

d. Microarray data

\section{Text AND page number from manuscript}

Page21. EGAS00001003634

Deposition is strongly recommended for many other datasets for which structured public repositories exist; more details on our data policy are available in the Guide to Authors. We encourage the provision of other source data in supplementary information or in unstructured repositories such as Figshare and Dryad. We encourage publication of Data Descriptors (see Scientific Data) to maximize data reuse.

18. If computer code was used to generate results that are central to the paper's conclusions, include a statement in the Methods section under "Code availability" to indicate whether and how the code can be accessed. Include version information as necessary and any restrictions on availability.
The code is deposited in GITHUB.

https://github.com/vermeeschlab/cfDNAhaplotyping

The code will become available under a license for research.

\footnotetext{
* If an error is shown on the PDF built in Editorial Manager after including this Reporting Checklist take the following steps:

1. Open the original filled GIM-reporting-checklist.pdf with Mac Preview App

2. Goto Tray $\rightarrow$ Export as PDF

3. Save as: at your desk (local computer) with a different file name

4. Close the app

5. In Editorial Manager: Edit the Submission which displayed Error or Incomplete Status and remove the file, then upload the new one, and again select to build the merged PDF
} 


\section{COLOR ARTWORK FORM}

This form must be completed for all papers. We will be unable to process your paper through to production until we receive instructions concerning color files. Please upload this form with your revised files.

JOURNAL: Genetics in Medicine

ARTICLE TITLE Non-invasive prenatal diagnosis by genome-wide haplotyping of cell-free plasma DNA

MANUSCRIPT NUMBER GIM-D-19-00723

CORRESPONDING AUTHOR NAME_ Joris Robert Vermeesch

Genetics in Medicine has charges for figures printed in color: $\$ 500$ for the first color figure and $\$ 250$ for each subsequent figure. Current ACMG members (excluding Student Members) who are first or senior/corresponding authors are exempt, ${ }^{*}$ as are authors who have opted for Open Access."

Please note that figures can appear online in color in the HTML version of your manuscript, and in black and white in the PDF/print version of the manuscript. Color figures will also be at the discretion of the editorial office.

Please check:

$\square$ Yes, my manuscript contains material that must be printed in color. I agree to pay the color charges in full and hereby authorize Nature Publishing Group to invoice me for the cost of reproducing color artwork in print.

$\square$ Yes, my manuscript contains material that should be in color in the online HTML version, but in black and white in the PDF/print version of the manuscript. No charges incurred.

Xes, my manuscript contains material that must be printed in color, but I have chosen Open Access for my article or am an ACMG member, so am exempt.

$\square$ No, my manuscript does not contain material that must be printed in color.

Which figures should be printed in color? (e.g. Figures $1 \mathrm{a}, 2,3 \mathrm{~b}$ )

College Member's Name:

Membership will be verified by the ACMG, and false claims will be liable for the full color charge rate. Signed completion of this form constitutes a full and total acceptance of the terms listed. College membership has no bearing on the review process at Genetics in Medicine, and papers from members and nonmembers alike will be given equal consideration.

Color figures will be set close to the citation and in the best possible position.

$20 \%$ VAT will be added to the total charge amount upon invoicing. This applies to all EU authors who do not provide a valid VAT number upon returning this form. Customers outside of the EU will not be charged VAT but local taxes will be added where applicable.

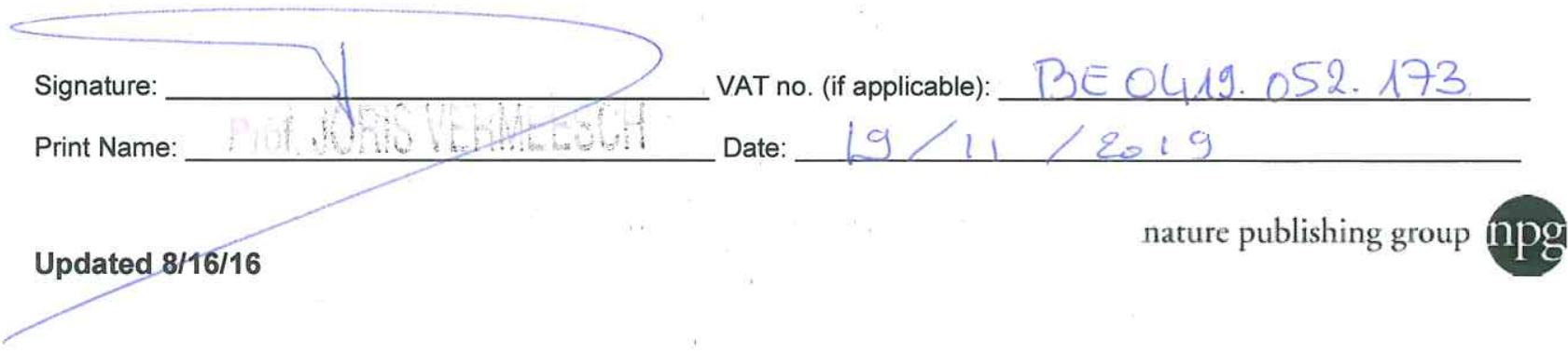

